\title{
Manuscript: Developmental variability drives mouse molar evolution along an evolutionary line of least resistance
}

Luke Hayden ${ }^{1,2}$, Katerina Lochovska ${ }^{3}$, Marie Sémon ${ }^{1,}$ Sabrina Renaud ${ }^{4}$, Marie-Laure

Delignette-Muller ${ }^{4}$, Maurine Vilcot ${ }^{1}$, Renata Peterkova ${ }^{5}$, Maria Hovorakova ${ }^{6} *$ and Sophie Pantalacci $^{1 *}$

\section{*corresponding authors}

1 Université de Lyon, ENS de Lyon, Univ Claude Bernard, CNRS UMR 5239, INSERM

U1210, Laboratoire de Biologie et Modélisation de la Cellule, 15 parvis Descartes, F-69007, Lyon, France

2 Université de Lyon, ENS de Lyon, Univ Claude Bernard, CNRS UMR 5239, Institut de Génomique Fonctionnelle de Lyon, 15 parvis Descartes, F-69007, Lyon, France 3 1st Department of Medicine, First Faculty of Medicine, Charles University and General University Hospital in Prague, U Nemocnice 2, 12808 Prague, Czech Republic 4 Université de Lyon, Université Lyon 1, CNRS, VetAgro Sup, UMR 5558, Laboratoire de Biométrie et Biologie Évolutive, Villeurbanne, France

5 Department of Histology and Embryology, Third Faculty of Medicine, Charles University, Ruska 87, 10000 Prague, Czech Republic 6 Department of Developmental Biology, Institute of Experimental Medicine, The Czech Academy of Sciences, Videnska 1083, 14220 Prague, Czech Republic 


\begin{abstract}
Developmental systems may preferentially produce certain types of variation and, thereby, bias phenotypic evolution. This is a central issue in evolutionary developmental biology, albeit somewhat understudied. Here we focus on the shape of the first upper molar which shows a clear, repeated tendency for anterior elongation at different scales from within mouse populations to between species of the Mus genus. In contrast, the lower molar displays more evolutionary stability. We compared upper and lower molar development of mouse strains representative of this fine variation (DUHi: elongated molars and FVB: short molars). Using a novel quantitative approach to examine small-scale developmental variation, we identified temporal, spatial and functional differences in tooth signaling centers between the two strains, likely due to different tuning of the activation-inhibition mechanisms ruling signaling center patterning. Based on the spatio-temporal dynamics of signaling centers and their lineage tracing, we show an intrinsic difference in the fate of signaling centers between lower and upper jaw of both strains. This can explain why variations in activation-inhibition parameters between strains are turned into anterior elongation in the upper molar only. Finally, although the "elongated" DUHi strain was inbred, first molar elongation was variable in adults, and we found high levels of intra-strain developmental variation in upper molar development. This is consistent with the inherent developmental instability of the upper molar system enabling the morphological variability of the tooth phenotype.

In conclusion, we have uncovered developmental properties that underlie the molar's capacity for repeated phenotypic change, or said differently, that underlie a "line of least resistance". By focusing on the developmental basis of fine phenotypic variation, our study also challenges some common assumptions and practices in developmental and evolutionary developmental biology.
\end{abstract}




\section{Introduction}

Evolutionary developmental biology postulates that developmental mechanisms confer specific variational properties on a trait, and can thereby channel its evolutionary trajectory. In extreme cases, a trait may repeatedly evolve similar phenotypes. Though this conceptual framework is central to evo-devo, it lacks cohesive supporting evidence. Only rarely the different levels of variation are bridged, from developmental variation to adult variation, and from variation between individuals to variation between populations or species. In this study focused on mouse molar teeth, we bridged these levels and reveal particularities of the developmental system that explain the morphological variation produced and its repeated appearance.

The idea that developmental mechanisms may channel and even direct the evolution of phenotypes is central to evo-devo (Brakefield, 2006, 2011; Hendrikse et al., 2007). It relies on the concept that developmental mechanisms bias the direction and the amount of variation available to both natural selection and neutral drift. This was recognized early, mainly under the term of "developmental constraints" (Gould and Lewontin, 1979; Smith et al., 1985) and studied from different viewpoints.

In the field of quantitative genetics, the analysis of phenotypic variation in crosses provides the direction of the genetic correlation between the different traits characterizing a shape. It was found that the direction of the genetic correlation between traits can match the direction of phenotypic variation within species, that itself matches the phenotypic variation between divergent populations or species. This suggested that phenotypic evolution happens along "genetic lines of least resistance"(Schluter, 1996). Because the structure of the genetic correlations itself also match over long time spans (eg. the G matrix was found to be similar among distant species), these "genetic lines" are thought to reflect developmental constraints, more than the persistence of specific genetic variants. This finding was recovered in a number of models including the molars of murine rodents (Renaud and Auffray, 2013; Renaud et al., 2006, 2011).

The study of developmental systems in terms of their evolution also argues for a role of development in orienting morphological diversification (Sears, 2014; Smith et al., 1985), including in the tooth model. This is recognized under the more specific term of "morphogenetic constrains". The patterns of variation recovered following experimental perturbations of amphibian development (Alberch and Gale, 1985; Oster et al., 1988) or 
mouse molar development (Kavanagh et al., 2007) predicted the pattern of morphological variation seen among species. By experimentally manipulating the mouse tooth germ or tinkering with one or two parameters of a computational model of tooth morphogenesis, Harjunmaa et al. (2014) (Harjunmaa et al., 2014) have reproduced evolutionary transitions seen in the fossil record, implying that the same construction rules have constrained morphogenesis since early mammals.

Despite this long interest and recent advances, there is still active discussion about how much development really influences evolutionary trajectories (Laland et al., 2014; Smith et al., 1985). One difficulty is that the different levels of variation are rarely bridged in a single model: from variation in embryo to variation in adult, and from variation in populations to variation between well diversified species. Mouse molars represent a rare opportunity to construct such a bridge: the Mus genus is well diversified, with many instances of repeated evolution and well characterized trajectories of phenotypic variation in molar shape.

Moreover, molar development is well known in the laboratory mouse.

In the $M u$ s genus, the main variation in first molar shape is between narrow and broad molars. This corresponds to a line of least resistance in the larger group of murine rodents (Old World mice and rats): the same main direction of phenotypic variation is recovered within populations of different murine rodents (e.g. house mouse and wood mouse populations) and at higher taxonomic levels (e.g. between Mus species or other murine species) (Renaud et al., $2009,2011)$. This trend is found in both lower and upper molars. On top of that, in some species or populations, the upper molar only tends to elongate, specifically from its anterior part which may even form an additional small cusp (Misonne, 1969; Renaud et al., 2011). This additional cusp is especially common in the Mus genus (yet occasionally seen in other murine species (Misonne, 1969)). For example, it is especially marked in some species of the Mus (Nannomys) subgenus, and also repeatedly seen in diverse house mouse populations ((Renaud et al., 2011), see later Figure 1). In particular, it evolved independently in many Mus (Mus) musculus domesticus island populations (e.g. on several Corsican islands, Marion Island (Renaud et al., 2011), Orkney islands (Ledevin et al., 2016; Renaud et al., 2018), as well as on Kerguelen and the Canary islands, S. Renaud, (Ledevin et al., 2016)). Interestingly, anterior elongation is found associated with increased body size in domestic mouse populations (Renaud et al. 2011), being trapped on island (and following "Foster's rule", or "island rule" where small mammals become gigantic) or in cold environments. In conclusion, 
bioRxiv preprint doi: https://doi.org/10.1101/818484; this version posted October 24,2019 . The copyright holder for this preprint (which was not certified by peer review) is the author/funder. All rights reserved. No reuse allowed without permission.

two intermingled lines of least resistance seem to act here: one acting on both first molars, and the other one acting on the upper molar only, involving its anterior elongation. 

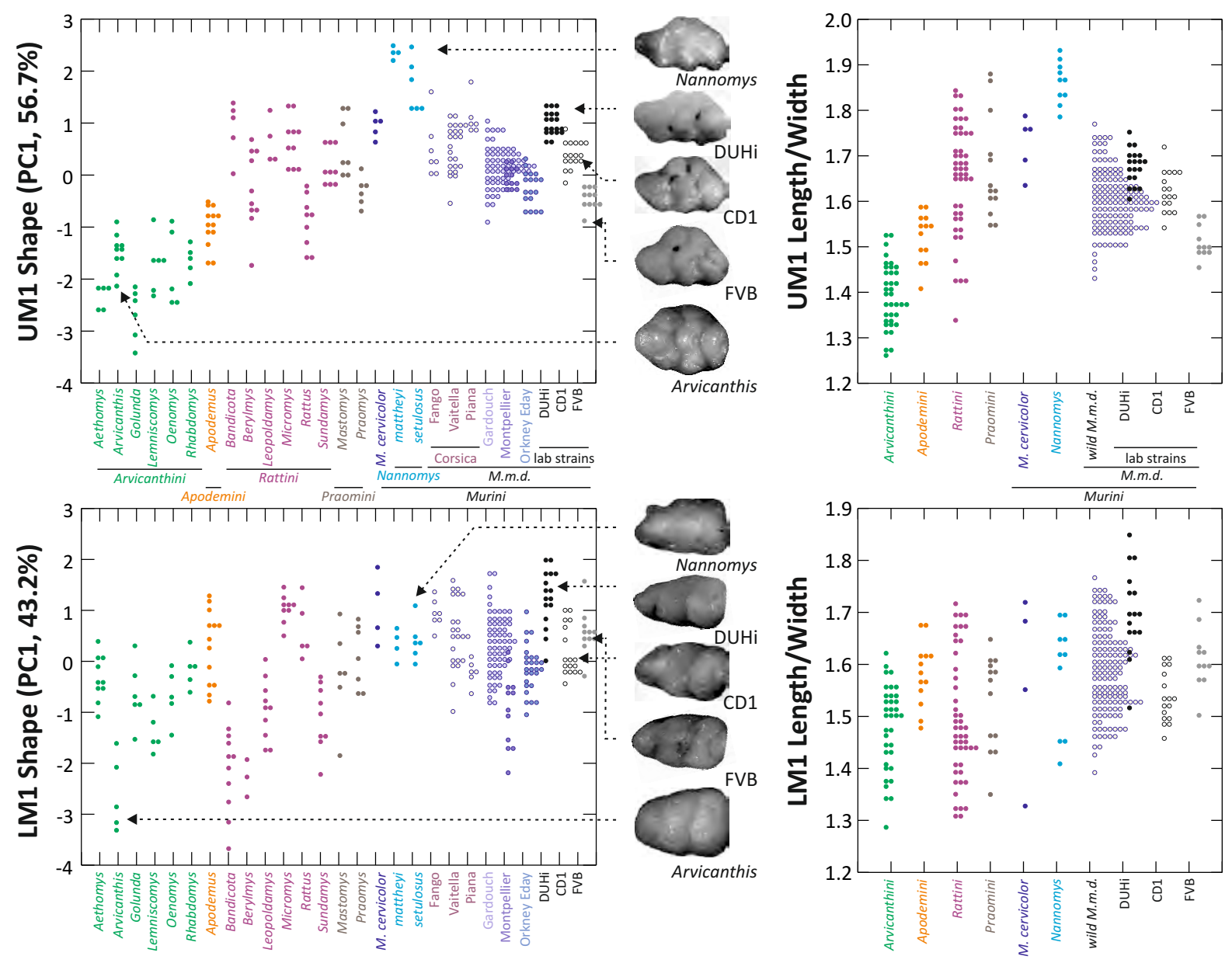

\section{Figure 1: Morphological variation in murine first molar shape, based on 2D outlines}

Morphological variation of the first molar, both upper and lower, examined in individuals from several murine species, including mouse natural populations of the house mouse Mus musculus domesticus (M.m.d) and three mouse strains (Mus musculus). The left panel shows the first axis of a principal component analysis of 2D outline descriptors (Fourrier coefficients). The right panel shows the length/width ratio of the molar (measures were taken on the same individuals as in the left panel, but they were grouped according to the phylogenetic groups shown in the upper left panel). Tooth images in the middle represent an example of the strain or species indicated, with arrows from each pointing to the point for that individual on each graph. UM1 - upper first molar; LM1 - lower first molar. 
bioRxiv preprint doi: https://doi.org/10.1101/818484; this version posted October 24,2019 . The copyright holder for this preprint (which

was not certified by peer review) is the author/funder. All rights reserved. No reuse allowed without permission. 
Molar development is well known (Balic and Thesleff, 2015; Peterkova et al., 2014), so that in a previous study, we had put forward some hypothesis for the developmental basis of this line of least resistance characteristic of the upper molar (Renaud et al., 2011). During molar development, signaling centers, called "enamel knots", are positioned in the epithelium by activation-inhibition mechanisms, and determine the location where the crown, or later the cusps, form (Jernvall et al., 1994). Though murine rodents lack premolar teeth, structures found transiently in mouse embryos are thought to correspond to their primordia, each with their own signaling centers. The primordium known as "R2" has been linked to the last premolar (Lesot et al., 1998; Peterkova et al., 1996; Peterková et al., 2002; Prochazka et al., 2010; Viriot et al., 2002). In the lower jaw, the R2 signaling center interacts in a complex manner with the signaling center of the first molar (Lochovska et al., 2015; Prochazka et al., 2010; Sadier et al., 2019). In CD1 mice, R2 and early M1 signaling center transiently co-exist before fusing to form the mature M1 signaling center known as the pEK (Lochovska et al., 2015). From an early cap stage, the R2 primordium becomes thus integrated into the lower first molar.

Although a similar R2 bud is also present in the upper jaw (Lesot et al., 1998; Peterkova et al., 1996), its developmental relationship to the upper first molar is less clear. It is not incorporated during the initial stage of molar cap development, as seen for its lower counterpart (Peterkova et al., 2006). Recently, we have shown that the distance between R2 and M1 signaling centers is larger in the upper jaw, and the two signaling centers do not fuse at the cap stage (Sadier et al., 2019). The anterior position of R2 and the difference between lower and upper R2, make these structures excellent candidates to explain that the upper molar, and not its lower counterpart, evolves so repeatedly towards anterior elongation (Renaud et al., 2011).

To get insights in the developmental basis of this line of least resistance, we chose two mouse strains reflecting the above-mentioned evolutionary variations: a "broad and upper-short" versus a "narrow and upper-elongated" strain. We looked for developmental variation in the dynamics of R2 rudimentary buds between strains and jaws, but also within strains, asking whether the upper molar developmental system is highly variable, as would be predicted by the evolutionary variation. 


\section{Materials \& Methods}

\section{Animal husbandry and ethical commitment}

DUHi mice were raised at the PBES; cryopreserved embryos had been obtained from MRC Mary Lyon centre, Oxfordshire, UK. FVB and CD1 mice were purchased from Charles River company.

C57BL/6 mice carrying tamoxifen-inducible Cre fused with the Shh allele (B6.129S6$\mathrm{Shh}<\operatorname{tm} 2(\mathrm{cre} / \mathrm{ERT} 2) \mathrm{Cjt}>/ \mathrm{J}$ ) and Cre recombinase-sensitive transgenic mice (B6.129S4Gt(ROSA)26Sortm1LacZSor/J) containing LacZ (beta-galactosidase) inserted into the Gt(ROSA)26Sor locus were used for the cell fate tracing study. The breeding pairs were purchased from the Jackson Laboratory (Maine, USA). The mice were genotyped using the Jackson Laboratory's protocols.

This study was performed in a strict accordance with European guidelines (2010/63/UE). It was approved by the CECCAPP Animal Experimentation Ethics Committee (Lyon, France; reference ENS_2014_022), by the Professional committee for guarantee of good lifeconditions of experimental animals at the Institute of Experimental Medicine IEM CAS, Prague, Czech Republic) and by the Expert Committee at the Czech Academy of Sciences (permit number: 027/2011).

\section{Morphometric analyses}

First upper and lower molars of a set of adult mice from the strains DUHi (19), FVB (11) and CD1 (17) were pictured using a Leica MZ 9.5 stereomicroscope. These teeth were compared with the variation observed within the murine rodents (Murinae). Their morphological diversity was documented by a set of specimens from the Museum National d'Histoire Naturelle (Paris, France) covering the main divisions of the group (Lecompte et al. 2008): Rattini with Rattus whiteheadi (5), Micromys minutus (9), Berylmys sp. (4), Leopoldamys sabanus (10), Bandicota indica and bengalensis (11) and Sundamys muelleri (9); Arvicanthini with Golunda ellioti (6), Lemniscomys barbarus (6), Oenomys hypoxanthus (5), Rhabdomys pumilio (5), Arvicanthis niloticus (5) and Aethomys chrysophilus and namaquensis (10); Praomyini with Mastomys chrysophilus (6) and Praomys tullbergi (7); Murini with Mus cervicolor (5), Nannomys setulosus (6), and Nannomys mattheyi (4). Apodemini were represented by Apodemus sylvaticus (13; data from Renaud et al. 2009). The sampling was completed with wild house mouse (Mus musculus domesticus) samples, documenting the continental and insular variation: Gardouch, France (68), Montpellier, France (13); Eday, 
Orkney, United Kingdom (18), Fango, Corsica, France (7), Vaitella, Corsica, France (24) and the islet Piana off Corsica, France (7) (data from (Ledevin et al., 2016; Renaud et al., 2011)). Maximal length and width were automatically extracted, together with 64 points along the outline, using the image analysing software Optimas 6.5.

The morphological variance in the total sample, including laboratory strains and wild species of murine rodents (200 specimens, with 198 first upper molars (UM1) and 192 first lower molars (LM1)) was summarized using principal component analyses (PCA) (one for the upper and one for the lower molars) on the variance-covariance matrix of the shape coefficients delivered by an outline analysis of the 2D occlusal surface (see (Renaud et al., 2009, 2011)). Fourteen variables were considered for both the first upper molar (UM1) and the first lower molar (LM1); all were standardized by the size of the respective tooth and corresponded to shape only. Maximum length and width of the outline were also measured and allowed the estimation of the overall elongation of the tooth (Length/Width ratio).

\section{Embryo harvesting and staging}

Mouse females were mated overnight and the morning detection of a vaginal plug was taken as proof of coitus, noon being taken 0.5 days post coïtum (dpc). We used a different day/night regime 12 hours apart to obtain embryos every half day. Pregnant females were sacrificed via cervical dislocation and embryos were harvested on ice and weighted.

\section{Dental epithelial dissociation}

Embryos were dissected in Hank's medium to separate upper from lower molars and then treated in Dispase II (Roche) $10 \mathrm{mg} / \mathrm{mL}$ at $37^{\circ} \mathrm{C}$ for 1 to $2 \mathrm{~h}$, depending on embryonic stage. Dental epithelium was then carefully removed and fixed overnight in paraformaldehyde (PFA) 4\%.

\section{In situ hybridization}

Shh probes were transcribed from a plasmid (described by (Echelard et al., 1993)), by means of in vitro transcription with the incorporation of digoxigenin-ddUTP, using a premixed DIG RNA labelling mix (Roche). In situ hybridisation was performed with a conventional protocol. The antibody utilised was an anti-DIG antibody coupled with alkaline phosphatase (Roche); the chromogenic substrate used was BM Purple ready-to-use NBT/BCIP (Roche). 
Samples were examined with Leica M205 stereomicroscope. Images were taken with the Leica Application Suite 4.1 software package.

\section{Modelling embryonic age}

In order to provide a measure of embryonic age, we built a model that could estimate the embryonic age from the body weight and dpc, taking into account intra and inter litter variations and applying a correction effect for the latter. This model and its construction are described in detail in Supplementary Methods. The code is available on: https://github.com/msemon/cdpc

\section{Sample classification and scoring}

The developmental state of all samples was assessed by combining four separate developmental criteria. Two are related with Shh expression: 1) Shh expression in the R2 (rudimentary premolar) zone, 2) Shh expression in the M1 (first molar) zone, and two are purely morphological criteria:3) the bud-cap transition and 4) the appearance of a protrusion, visible in the dissociated epithelium at the site of the R2 signaling center, but only in the latest stages of Shh signaling and after cessation of Shh signaling. For each criterion, the samples were scored as one of 2 or 3 states. This scoring system is summarized in Supplementary Table 1. Two tables with all analyzed embryos, with their weight, age in cdpc, information on litter and scored characteristics, and the code used to analyze these data is provided on github: https://github.com/luke-hayden/dvpap/devstate

\section{D reconstruction}

Dissected tooth germs were fixed overnight in 4\% PFA and dehydrated through a methanol series. In toto immunolocalisation protocol was adapted from (Ahnfelt-Rønne et al., 2007). Following incubation in methanol added with H202 5\% and DMSO 10\% for 4 hours at room temperature, they were rehydrated, blocked with serum and incubated successively with an anti-laminin5a antibody (overnight,1/800, kind gift from Jeff Miner,(Miner et al., 1997)) and a Dylight 549 conjugated Donkey Anti-rabbit antibody (overnight 1/200, Jackson immunoresearch). Following dehydration, they were clarified and mounted in BABB as described in (Yokomizo and Dzierzak, 2010). They were imaged with a Zeiss LSM710 confocal microscope at the PLATIM (Lyon, France). The basal membrane labelled by the antibody was delineated semi-manually and reconstructed with the AMIRA software. 


\section{First molar germs RNA Sequencing}

RNA-Seq were made on twelve carefully dissected embryonic lower and upper first molar germs, from DUHi (embryo weight: 196, 219 and $239 \mathrm{mg}$ ) and FVB (195, 215 and $233 \mathrm{mg}$ ) strains. Following dissection in culture medium, tooth germs were stored in RNA later at $20^{\circ} \mathrm{C}$. RNA was extracted with RNAeasy micro kit (Qiagen), and controlled with Q-bit (Invitrogen) and Tapestation (Agilent technologies). RNA-Seq samples were prepared following the TruSeq ${ }^{\circledR}$ RNA Sample Preparation v2 Guide, starting from 100ng of total RNA of top quality (RINe $>9.5)$. Sequencing was performed with the Illumina HiSeq 4000 system (single-end 50-bp reads).

\section{Detection of differentially expressed genes with DESeq2 package.}

Reads were then mapped to the mouse genome using Kallisto ((Bray et al., 2016), version 0.44.0, options -1 200, -s 20). The reference cDNA sequences and annotation files for $M$. musculus are based on C57B6 strain. They were collected from Ensembl 88 (10 5129 cDNAs, (Zerbino et al., 2018), GRCm38). Reads were independently mapped to the FVB/NJ strain cDNAs, collected from Ensembl strains 94, using biomart (10 1520 cDNAs, strain FVB_NJ_v1, accession GCA_001624535.1). Tximport was used to import and summarize transcript-level estimates at gene level (version 1.6, (Soneson et al., 2016)). Differentially expressed genes were detected with DESeq2 (Love et al., 2014), version 1.18.1) with classical one-factor design, and using FDR significance threshold =0.05. 19202 genes are in common between FVB and reference strain C57B6 and have a MGI annotation. Out of these genes, 2234 genes $(11.6 \%)$ presented a significant difference of expression between the mapping on the reference strain C57B6 and the mapping on FVB strain (DESeq2, adjusted p-value $<0.05$, considering the mapping effect that is with 12 replicates). This is presumably a mapping artifact, due to the sequence divergence between mouse strains. These genes were removed, and the remaining 16,968 genes were retained for further analysis. 3619 genes were found to be differentially expressed between the two strains taking into account the jaw of origin (lower/upper) ( jaw + Strain). Processed data with statistics are provided in Supplementary File 1. Raw and processed data were deposited in NCBI Gene Expression Omnibus (GEO, accession number GSE135432;

https://www.ncbi.nlm.nih.gov/geo/query/acc.cgi?acc=GSE135432). The mapping data (by Kallisto, on each reference strain as discussed in the text), $\mathrm{R}$ source code and parameters are available on github https://github.com/msemon/trDUHi FVB 


\section{Comparison of BMP4 and Wnt pathway signaling activity in DUHi and FVB mice based on transcriptomic data}

We used a list from supplementary data published by O'Connell and colleagues (O'Connell et al., 2012) describing regulatory interactions for BMP4 and Wnt pathways in tooth epithelium and tooth mesenchyme at different developmental stages, by combining data mining with results of their own perturbation experiments. For BMP4 pathway, it describes up or downregulation (+: upregulated, -: downregulated, o: no change) of presumptive target genes upon BMP4 treatment (perturbation +) or BMP4 knockout (perturbation -). For Wnt pathway, it describes up or downregulation of presumptive target genes upon inhibition of Gsk3b (i.e. Wnt pathway activation), Ctnnbl overexpression (i.e. Wnt pathway activation), Ctnnb1 knock-out (i.e. Wnt pathway inhibition), $D k k l$ overexpression (i.e. Wnt pathway inhibition), Lefl knock-out (i.e. Wnt pathway inhibition), or treatment with different Wnts. First, we checked if genes of this list were differentially expressed in the above analysis. For those that were DE, we compiled the O'Connell table to determine if the gene was a positive, a negative or not a target of the pathway. A gene that is upregulated upon pathway activation, or downregulated upon pathway inhibition was considered a positive target. A gene that is downregulated upon pathway activation, or upregulated upon pathway inhibition was considered a negative target. When data were conflicting between tissues (eg. positive target in epithelium, negative target in mesenchyme; 7 genes, e.g. $D l \times 1)$, the gene was excluded from the analysis, because in our analysis the whole tooth germ is examined. When data were conflicting between different sources (two genes: Egrl, Ptch1), we kept the result obtained by O'Connell et al. because 1) these are transcriptomic data and 2) most interactions described in the table are from this study only or are confirmed in this study, and thus the result obtained for this gene has more chance to be consistent with results for other genes in the table. The resulting table is shown in supplementary file 1.

\section{Fate mapping of $S h h$ expressing cells using X-gal staining}

The strain B6.129S6-Shh $<\operatorname{tm} 2$ (cre/ERT2)Cjt $>/ \mathrm{J}$ was reciprocally crossed with a reporter strain containing LacZ inserted into the Gt(ROSA)26Sor locus in order to mark the cell population expressing $S h h$ from the time of the tamoxifen injection into pregnant female mice. Pregnant female mice were injected intra-peritoneally with tamoxifen at E12.5 (when Shh is expressed in the R2 expression domain and early M1 expression is not yet apparent), E13.5 (when Shh expressing domain in R2 finishes its activity and early M1 signaling center 
starts to be apparent posteriorly) or at E14.5 (when only M1 signaling center express Shh). Tamoxifen was administrated in a dose of $0.225 \mathrm{mg} / \mathrm{g}$ of body-weight (Hayashi and McMahon, 2002). Such a concentration is not hazardous for pregnant mice or embryos and is sufficient for the fast activation of recombination. The embryos were harvested at $17.5 \mathrm{dpc}$, 72, 96 or 120 hours after tamoxifen application and beta-galactosidase activity was detected on whole embryos or dissociated epithelia of upper and lower cheek region. The X-gal (Sigma) concentration in the staining buffer was $3 \mathrm{mM}$. Samples with positive staining were post-fixed in PFA (4\%) overnight. After post-fixation, the samples were washed in PBS and photographed using a Leica MZ6 stereomicroscope equipped with a Leica EC3 digital camera (Leica Microsystems GmbH, Wetzlar, Germany). Data are summarized in Supplementary Table 2.

\section{Organotypic culture}

The upper molar region of 13.0 dpc FVB or DUHi embryos were dissected and cultured according to standard methods described in Kavanagh et al. 2009. Tooth culture was stopped after 40h and imaged using a Leica MZ6 stereomicroscope equipped with a Leica EC3 digital camera (Leica Microsystems GmbH, Wetzlar, Germany).

\section{Measurement of developmental variation}

Measuring developmental variation is a complex task; it requires that we can measure factors that change with time as development proceeds. If we consider how a developmental system proceeds along its trajectory, we will expect that it changes gradually over time, where a given sample is most similar to those at the closest time-points. However, where developmental variation is present, we expect to find pairs of samples that are of the same embryonic age but differ markedly in form. So, for each strain and for both upper and lower molars separately, we took all of our set of samples, determined their computed embryonic age (in ${ }_{c} \mathrm{dpc}$ ) and their developmental state, then took all possible pairs of samples where both members of the pair were close in age (less than 0.25 dpc difference). For each pair of samples, we can then compute a pairwise developmental distance: the distance between the two samples in terms of developmental state, computed as the sum of the score difference obtained for each 4 developmental criteria (Supplementary Table 1). For each strain for both upper and lower molars, we could then plot the distribution of these pairwise developmental distances. Finally, in order to compare total developmental variation between strains, we subjected these pairwise developmental state differences (between pairs of samples at $\left.{ }_{c} d p c\right)$ to 
a Wilcoxon rank-sum test. Using this method, we can measure the degree of developmental variation found in a set of samples and compare between strains. The code is provided on github: https://github.com/luke-hayden/dvpap/devstate.

\section{Measurement of developmental variation over time}

We first calculated pairwise developmental state distances for each sample in relation to all nearby samples, over computed embryonic time. Then to obtain developmental variation over time, we used locally estimated scatterplot smoothing (LOESS), a non-parametric regression method to plot a complex curve through many data points, weighting the contribution of data points according to their proximity to the point of estimation. The code is provided on github: https://github.com/luke-hayden/dvpap/devstate.

\section{Other Statistical Analyses}

The statistical significance of differences in the timing of developmental events was tested using logistic regression (embryo weight as a predictor of state, with strain as an additional preictive factor), examining changes in the scoring of a developmental criterion (four criteria scored, see previously) in relation to computed embryonic age. The statistical significance of differences in the sizes of various morphological features was tested using Student's t-test. Fisher's test was used to test differences in the relative rarity of a developmental state within a given window. The code is provided on github: https://github.com/lukehayden/dvpap/devstate. See also Supplementary Statistical Details.

All statistical analyses were carried out using the R statistical environment (R Core Team, 2015), version 3.2.3. Packages used included ggplot2 (Wickham, 2009), reshape2 (Wickham, 2007) and phytools (Revell, 2012). 


\section{Results}

It was noted long ago that two laboratory mouse strains displayed the elongated upper molar morphology with a small additional anteriormost cusp $(\mathrm{C} 3 \mathrm{H}, 101$ strain from Harwel, (Grüneberg, 1965)). In our effort to test the correlation between this morphology and a large body size, we found that the DUHi mice, an inbred strain that was established following artificial selection for increased body size (Bünger and Herrendörfer, 1994; Bünger et al., 1982), display narrow molars with elongated first upper molars, and an additional cusp in some individuals (Supplementary Figure 1). In contrast, the FVB mice, an inbred strain often used to maintain genetic modifications, display wide molars with short first upper molars. A representative sampling of FVB and DUHi tooth morphology is seen in Supplementary Figure 1.

\section{The variation between DUHi and FVB molar morphology follows the murine evolutionary line of least resistance}

In order to place these two strains within the context of natural variation in molar tooth shape, we compared the outline of first molars in a number of murine groups, including Mus musculus domesticus from the wild and three lab strains (two inbred strains: FVB, DUHi; one outbred strain CD1). We performed a principal component analysis (PCA) of outline descriptors (obtained from an outline analysis of the 2D outline, see methods) and a direct comparison of molar Length/Width ratio (Figure 1). For both the upper and lower molar, the first axis of the PCA contrasts broad with narrow outlines. Hence, this can be considered to be the most important aspect of the outline variation for both teeth, with a morphology ranging from short, compact and rounded teeth to long and narrow teeth. However, this variation is more pronounced for the upper molar (PCA UM1 $=62 \%$ instead of PC1 LM1 $=46 \%$ of variance) because it also involves a change focused at the anterior part of the tooth, and opposing short vs. anteriorly elongated UM1. This variation corresponds to the evolutionary trend seen repeatedly for the upper molar in the Mus genus: the anterior elongation that can even take the form of a small additional cusp (Renaud et al. 2011; Stoezel et al. 2013). The position of FVB and DUHi strains along the PC1 axis and length/width ratio indicate that the direction of variation between the two strains recapitulates the direction of variation seen in murine rodents as a whole (e.g. broader FVB molars versus narrower DUHi molars), and even more especially, the direction of variation seen in the upper molars of the Mus genus (short FVB versus anteriorly elongated DUHi upper molars). Indeed, the two strains are 
representative of extreme wild Mus musculus domesticus samples: DUHi teeth fall with the most elongated upper molars (e.g. samples from a very small Corsican island: Piana), while the FVB upper molars fall with the most "short and wide" molars (e.g. samples from the continent (Gardouch locality) or from a large Orkney island: Eday). This validates the choice of these strains, meaning that the next step was to examine the developmental basis of interstrain variation in lower and upper molars.

\section{Accounting for inter-strain timing differences and the dynamic nature of the system}

In order to allow the developmental trajectories of the developing molar teeth to be compared, embryonic samples were taken from a wide developmental window in the two strains in question. In the FVB strain, this period stretches from 12.5 days post coitum (dpc) to $16.5 \mathrm{dpc}$ and corresponds to Theiler stage 20-24. During this period, the dental epithelium progressively invaginates into the mesenchyme along the antero-posterior jaw axis, forming an epithelial ridge. Anteriorly in this elongating ridge, the epithelium locally enlarges and gives rise to a wide $\mathrm{R} 2$ bud (a rudiment of the suppressed premolar) and the signaling center of the R2 (rudimentary premolar) appears. As the ridge further elongates more posteriorly, taking the characteristic bud shape seen on frontal sections, the M1 (first molar) signaling center forms. Then, the dental epithelium spreads around the M1 signaling center (transition to the cap stage) and, later on, the secondary enamel knots start to form (bell stage): this will determine the formation of the molar crown with its cusps. For each strain, multiple litters were sampled every half day, ensuring even coverage due to the slight variation in developmental stage within litters.

To allow comparisons between jaws and strains, we proceeded to examine development in a quantitative fashion, taking account of the dynamic nature of the system. For each sample, four key characters were scored for two to three possible states (characters were: the expression of $S h h$ at the premolar R2 signaling center, the expression of $S h h$ at the M1 signaling center, the progression of the bud-cap transition at the level of M1, and the protrusion of the dental epithelium at the level of R2, Supplementary Table 1).

Taking account of the dynamic nature of the system required a numeric estimation of the embryonic age of embryos (i.e. an age reflecting the progress in embryogenesis). In a given strain, age estimated from the calculation of days-post-coitum provides a very bad estimation, because of sharp differences between litters (standard range $+/-0$ to 0.5 days variation in embryonic age, notably due to difference in fertilization and implantation time) and because it does not take into account the slight variation in developmental stage within litters (standard 
range from 0 to a quarter day). A combined "age/weight" staging has been recommended previously (Peterka et al., 2002). Embryonic body weight provides a much better numeric estimation than age in dpc alone (Pantalacci et al., 2009; Peterka et al., 2002). This estimation is especially reliable within litters (at the stages examined here) but less reliable between litters, presumably because the nutritional status differs from one pregnancy to the other, causing embryos of similar embryonic age to have smaller or larger body weight. Age in dpc can help to correct for this, since embryos with similar body weight and similar harvesting age will have higher chance to have reached similar developmental stage (similar embryonic age), while embryos with similar body weight but different harvesting age in dpc will have higher chances to be at different embryonic age. On top of these intra-strain differences, there are differences between strains: embryos sampled at a given number of days post-coitum were not of the exact same embryonic age range in each strain.

Therefore, in this study, we devised a Bayesian modelling approach to compute a strainspecific embryonic age for each embryo (later called ${ }_{c} d p c$ ), based on embryo body weight and age in dpc, taking into account intra and inter litter variation in each strain. Such a model learns from the data, and could also take into account strain differences without imposing $a$ priori assumptions. Because the model does not comprise information on actual stage differences and we estimate ${ }_{c}$ dpc for each strain, it will not correct for developmental stages differences between strains, but instead reveals them by providing a time framework, the computed age (e.g. cap transition occurs at earlier ${ }_{c} d p c$ in FVB). The detailed model and the script are provided in supplementary methods. An important stochastic term in the model corresponds to the part of the inter litter variation of the body weight due to pregnancy, for which we explored two values (Figure of Supplementary Methods). The first one, which we consider realistic, corresponds to a maximum effect on weight of $20 \mathrm{mg}$ for a $200 \mathrm{mg}$ embryo. The second one, which we consider an extreme upper bound, corresponds to a maximum effect on weight twice as important (40 mg for a $200 \mathrm{mg}$ embryo). The computed embryonic ages (next called ${ }_{c} \mathrm{dpc}$, for computed days post coïtum) presented in the main text have been estimated using the realistic parameter, but the results are qualitatively robust using the upper bound parameter (Supplementary Methods, Figures 2-9). This demonstrates that our results are robust to noise in embryonic age estimation. We also used embryonic age directly estimated from body weight (a simplification of the previous model, similar to what we used in our previous studies, (Pantalacci et al., 2009)). All the results shown in this study were robust in relation to these estimations, although they differ slightly (Supplementary Methods Figures 2-9). In the next paragraphs, the results obtained for upper molar are mostly presented 
in the main figures and results for lower molar will be found in the supplementary material.

\section{The range of possible developmental states and the developmental trajectory taken} differs between strains, especially for the upper molar

We proceeded to compare the DUHi and FVB lower and upper developmental systems. In both jaws and strains, we see $S h h$ expression at the signaling center of the R2 bud (Figure 2 and Supplementary figure 2 A-D). This expression then fades away (Figure 2 and Supplementary Figure 2 E-H). A second spot of expression appears, which represents the early M1 signaling center (Figure 2 and Supplementary Figure 2 I-L). As development proceeds to the cap transition stage, the M1 expression zone increases in size (Figure 2 and Supplementary Figure 2 Q-T and 3) to form a "mature M1 signaling center" (differences between lower and upper jaw in this process will be emphasized later), and the tooth continues to develop (Supplementary Figure 3 and 4). A simple examination of embryonic series suggested differences in the dynamics of the signaling centers (Fig2, e.g. see I-J versus K-L). In the upper jaw of DUHi mice, we frequently see the co-occurrence of the fading R2 spot with a distinct M1 spot (Figure $2 \mathrm{M}-\mathrm{T}$ and Supplementary Figure $2 \mathrm{M}-\mathrm{T}$ ). 


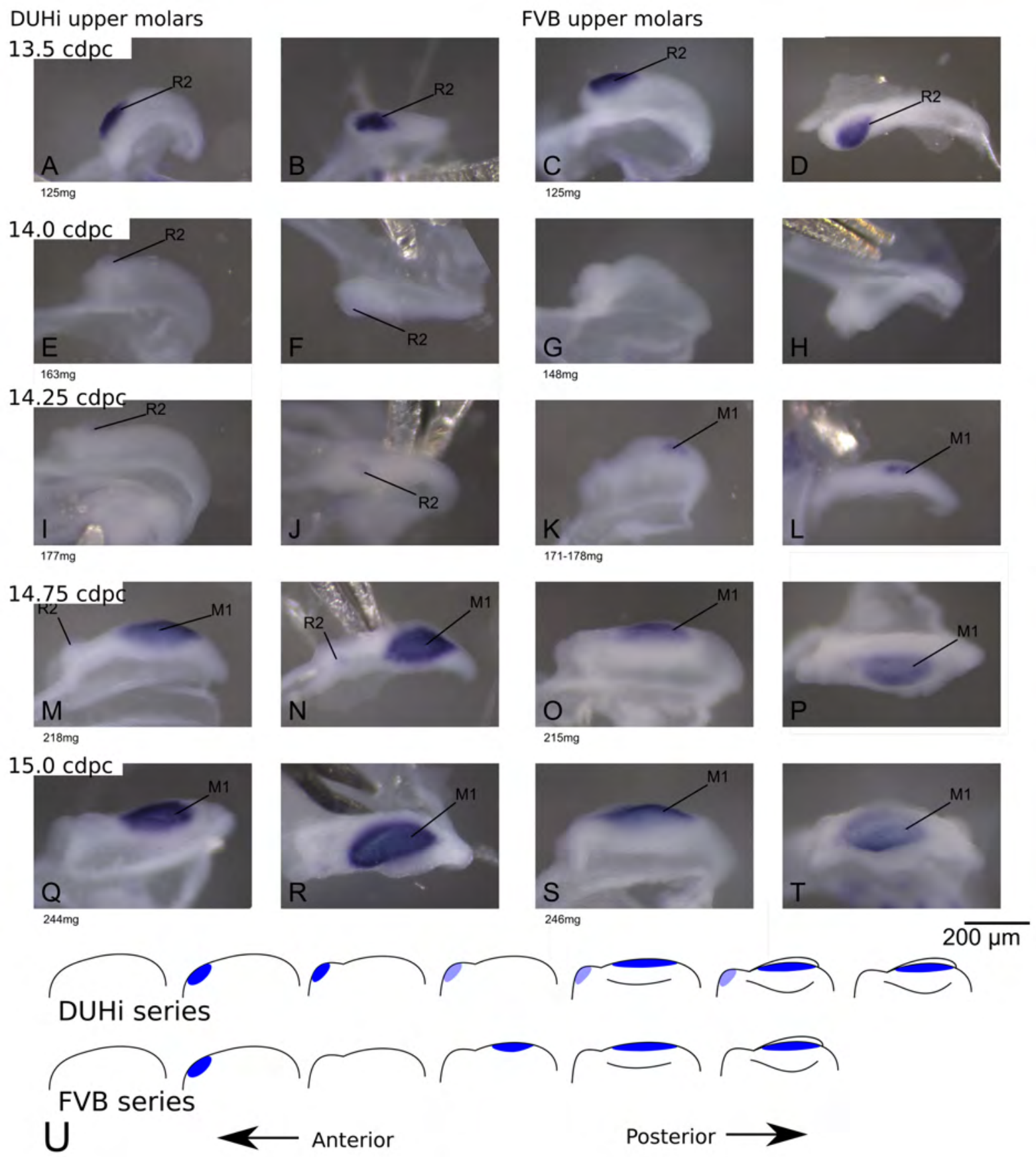

Figure 2: Comparative early molar tooth development in upper molar epithelia of DUHi and FVB mice

Dissociated upper molar dental epithelia epithelia of DUHi (left-most two columns) and FVB embryos (right-most two columns), marked for Shh expression with in situ hybridisation. Samples represent a developmental series of early molar development, corresponding to $12.5 \mathrm{dpc}$ to $15.0 \mathrm{dpc}$ in FVB and 13.0dpc to $15.5 \mathrm{dpc}$ in DUHi. Embryo weight is noted below each sample and its equivalent in computed embryonic age is noted above each row of samples. Two images of each sample, a side and a top view, are shown. 
To help illustrate these differences, we scored the four criteria previously defined (Supplementary Figures 5 and 6) and combined these scores to compute the developmental state of each sample (Figure 3 and Supplementary Figure 7). In theory, this yields 54 possible total states of the dental epithelium, which can be conceptualized as a theoretical "developmental space" through which each individual will move as it develops. However, not all of this space is occupied; the number of states observed was much smaller than the mathematical maximum theoretically possible (11 states for the upper molar, Figure 3; and 9 states for the lower molar, Supplementary Figure 7). A comparison of the distribution of developmental states seen in DUHi and FVB upper molar samples reveals that certain states are only present in one of these two strains. This is especially true for upper molar (3 DUHiexclusive states, 2 FVB-exclusive states) as compared with lower molar (1 DUHi-exclusive state, evidenced by only 2 samples). Indeed, within a given weight range (from 14.25-14.75 cdpc ;175mg to 225mg), all DUHi samples display a "DUHi-exclusive" state, while all FVB samples display an "FVB-exclusive" state. This corresponded to the period between the disappearance of the R2 signaling center and the maturation of the M1 signaling center. Therefore, the developmental differences between these two strains can be conceived of as each of the two strains following distinct trajectories through "developmental space". Moreover, these differences are especially marked in the upper molar. 


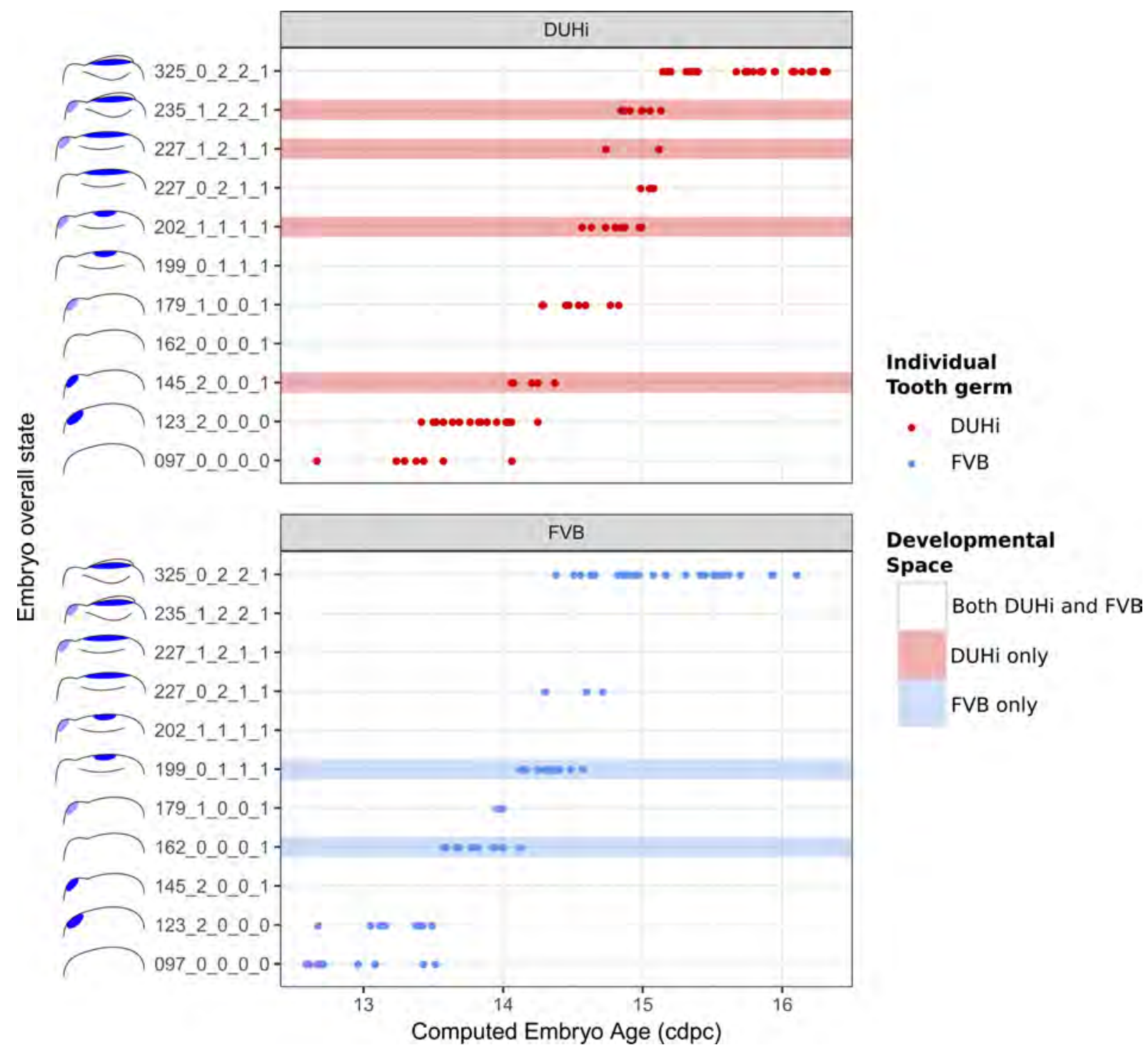

Figure 3: The range of possible developmental states differs between FVB and DUHi

\section{developing upper molars}

Temporal distribution of developmental state of the developing upper molar, produced by combining a value for each of the four scores for a given sample, based on criteria from Supplementary Table 1. Each of the developmental states observed are schematized by a cartoon and ordered according to the average embryonic weight of the samples within that group. They are named with this weight, followed by the value of the four scores (eg. 097_0_0_0_0, means average weight $97 \mathrm{mg}, 0$ value for the four scores). Exclusive developmental states are highlighted according to whether they are found in DUHi only (red) or in FVB only (blue). The temporal axis is given by computed embryo age (cdpc). The dental epithelium is oriented with anterior part to the left. Scale bar $=200 \mu \mathrm{m}$. Developmental progression is summarized in schematic form below $(\mathrm{U})$. 


\section{The premolar signaling center persists longer in DUHi than in FVB upper jaws}

We then compared the timing of developmental events between the two strains. In upper molars (Figure 3 and Supplementary Figure 5), the R2 expression zone persists longer in DUHi than in FVB (logistic regression: presence of R2 signaling center activity in relation to computed embryo age, $\mathrm{p}<1.10 \mathrm{E}-8$, see Supplementary statistical details 1). The R2 and M1 signaling center frequently co-occur in DUHi, and co-occurence of R2 and a mature (large) M1 signaling center was only seen in DUHi upper molars. In contrast, early M1 signaling center was seen without a R2 signaling center only in FVB mice. A logistic regression also revealed a statistically significant difference in the timing of the appearance of the M1 spot, which appears later in DUHi than in FVB embryos $(\mathrm{p}<0.01$, see Supplementary statistical details). The longer persistence of R2 signaling center and later appearance of the early M1 signaling center in the upper jaw of DUHi mice were associated with a more prominent R2 bud, as seen in dissociated epithelium (Figure 2, Figure 4-F) but also in 3D reconstructions of tooth germs (Figure 4 A-D).

In the lower jaw, we observed a similar tendency, with R2 and M1 signaling centers occasionally coexisting only in DUHi, but never in FVB (Supplementary Figures 6 and 7). In both strains, the lower R2 signaling center was ultimately part of the mature M1 signaling center known as the pEK (Supplementary Figure $2 \mathrm{R}$ and P), as shown for other mouse strains (Lochovska et al., 2015). 

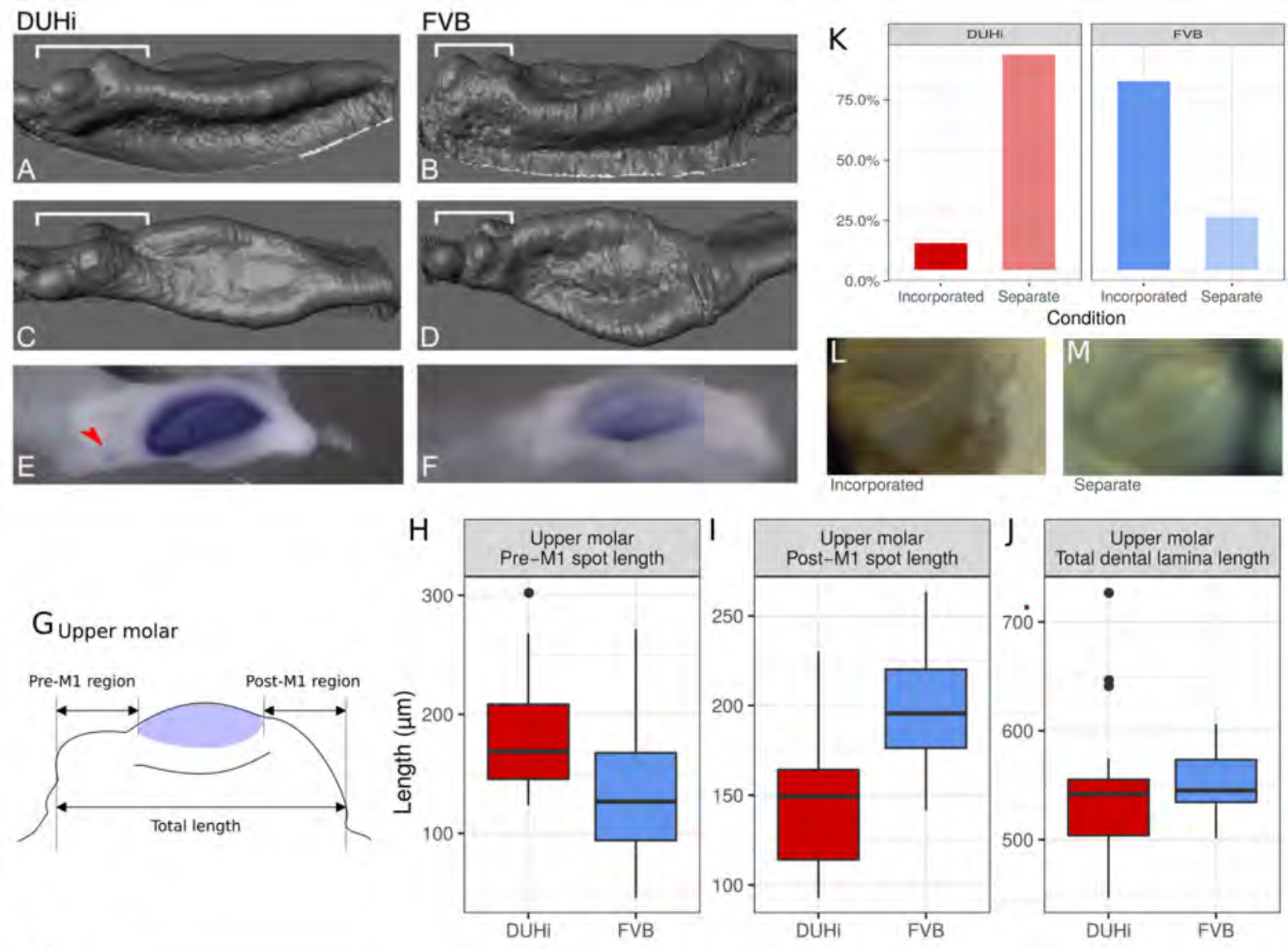

N

잉
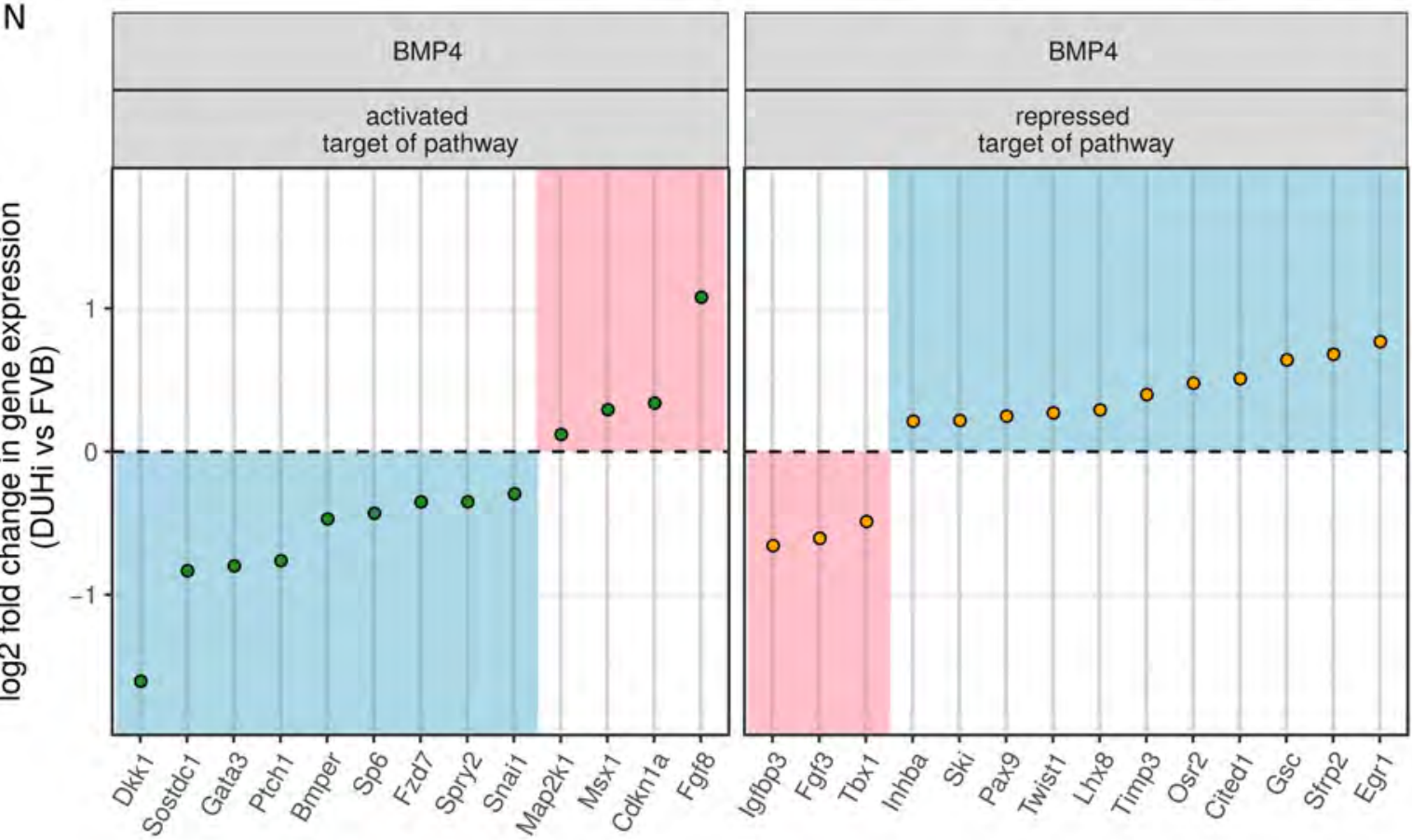

pathway less active in $\mathrm{DUHi}$

pathway more active in $\mathrm{DUHi}$

Figure 4: FVB and DUHi strains differ in the balance of activation-inhibition

\section{mechanisms}


(A-F) Comparative 3D morphology of the epithelial part of developing upper molars in DUHi (A, C) and FVB (B, D) strains at cap transition. A, B are side views and C, D upper views. The variable anterior region including R2 rudimentary bud is denoted by the bracket. Expression of Shh at the same timepoint is shown below for DUHi (E) and FVB (F), for comparative purposes. The red arrowhead in E points to R2 signaling center. In F, the faint staining anterior to the mature M1 signaling center might correspond to re-expression of Shh in cells that formed R2 signaling center. (G-J) panel G shows the three measurements taken from all epithelial samples of a computed age of $14.25-15 \mathrm{cpdc}$ (between 180 and 250mg weight), comparing DUHi with FVB samples. The measures were taken between the anterior or posterior limit of dental epithelium and the anterior or posterior limit of the M1 signaling center, as shown on the cartoon. Boxplots H-J show the results in upper molars (see supplementary for lower molars). Pre-M1 region and Post-M1 regions are significantly different in DUHi versus FVB mice ( $\mathrm{t}$-test; $\mathrm{p}<0.01$, see Supplementary statistical details 1 ). (K-M) the developing molars of DUHi and FVB mice react differently when cultured in vitro at $13.0 \mathrm{dpc}(\mathrm{p}=0.015$ in an exact Fisher test, see Supplementary statistical details): R2 bud tends to form a clear individualized bud (M) in most DUHi tooth cultures ( $\mathrm{n}=18)$, whereas a single developing tooth $(\mathrm{L})$ is seen in most FVB tooth cultures $(n=18)$. (N) Target genes of the Bmp4 pathway differentially expressed between the two strains at the cap transition. Differential expression analysis was performed on both lower and upper molar samples, taking molar type into account in the statistical treatment by DEseq2. Genes were classified as targets activated or repressed by the pathway based on (O'Connell et al., 2012). The $\log 2$ fold change in DUHi as compared to FVB is shown (positive: expression level increased in DUHi tooth germs; negative: expression level decreased in DUHi tooth germs). Depending if the gene is an activated or a repressed target, and is increased or decreased in DUHi tooth germs, it may suggest that the pathway is more active (pink) or less active (blue) in DUHi tooth germs.

\section{FVB and DUHi strains differ in the balance of activation-inhibition mechanisms}

The balance in activation-inhibition mechanisms has been shown to rule the fate of the R2 rudimentary bud (Klein et al., 2006; Peterkova et al., 2009; Sadier et al., 2019). The finding that R2 is larger and longer lived in DUHi mice suggests that this balance differs between the two strains.

A prediction is that the positioning of the M1 signaling center should differ between the two strains. Our measurements revealed that the M1 signaling center was shifted posteriorly in DUHi mice: the pre-M1 signaling center region was longer in DUHi than in FVB samples (Figure $4 \mathrm{H}$, t-test, $\mathrm{p}<0.01$ ), while the post-M1 signaling center region was shorter in DUHi upper molars than in FVB (Figure 4I, t-test, $\mathrm{p}<0.01$; see Supplementary statistical details). No statistically significant differences in total dental epithelium length was found (Figure 4J). In the case of the lower molars (Supplementary figure 8, see Supplementary statistical details), no statistically significant inter-strain differences emerged. 
Another prediction is that the two strains, having different parameters for the balance between activation and inhibition, should react differently to the same perturbation of activationinhibition mechanisms. Simply dissecting and culturing teeth ex vivo is known to provide such a perturbation, resulting in incisor germ splitting (Haara et al., 2012) or in a partial rescue of the lower R2 rudimentary bud (Sadier et al., 2019). Culturing upper molars, we have occasionally observed such a rescue of the upper $\mathrm{R} 2$ bud which starts to form an independent tooth cap (Figure 4M). This occurred much more frequently in DUHi than in FVB mice (Figure $4 \mathrm{~K}-\mathrm{M}$; Fisher exact test; $\mathrm{p}=0.015$ ). This is consistent with R2 being partially rescued in the DUHi mice.

Finally, we looked for gene expression differences between the two strains that would be consistent with a difference in the balance of activation and inhibition. For that, we generated transcriptomes of lower and upper molar germs of FVB versus DUHi mice at the time when R2 and M1 signaling center co-exist in DUHi mice. We found a large number of differentially expressed genes (see Supplementary Text 1 for detailed results, data available in Supplementary Table 2). Among them, we identified two genes, Spry2 and Sostdc1 (also known as Ectodin), whose knock-out causes the formation of a premolar-like tooth (Ahn et al., 2010; Cho et al., 2011; Klein et al., 2006). Where it was specifically investigated, this premolar tooth was demonstrated to arise from R2 revival (Ahn et al., 2010; Klein et al., 2006; Peterkova et al., 2009). The downregulation of these two genes in DUHi lower and upper molar samples (Supplementary Figure 9) could thus help the partial rescue of R2 bud in this strain. We then focused on the Wnt and BMP4 pathways that have been shown to be key for tooth formation (O'Connell et al., 2012). Because the activation-inhibition (A-I) balance involves many genes with complex regulatory feedbacks within and between these two key pathways (and other pathways), we did not expect a change in the A-I balance to shift all target genes in a consistent direction. Rather, we expected to find a different equilibrium, with genes changed in both directions, but that may collectively indicate greater or instead weaker activation of these pathways in the DUHi mice. For the BMP4 pathway, there were 20 genes in favor of weaker BMP4 activation in DUHi (e.g. summing activated targets that are upregulated with repressed targets that are downregulated, see supplementary text 1 for more detail and figure $4 \mathrm{~N}$ ) versus only 7 genes in favor of greater BMP4 activity (e.g. summing activated targets that are downregulated with repressed targets that are upregulated). This twofold difference is significant ( $\mathrm{p}=0.01$ in a $\chi^{2}$ test). For the Wnt pathway, we found no trend with 23 genes in favor of weaker Wnt activity in DUHi and 20 genes in favor of greater Wnt 
activity (Figure 4N). Intriguingly, this included 4 feedback inhibitors of the Wnt pathway upregulated in DUHi mice (Axin2, Kremen1, Osr2; Sfrp2) and 3 feedback inhibitors downregulated in DUHi (Dkk1, Wif1, Sostdc1). Finding these major regulators of Wnt activity in tooth development differentially expressed suggests that Wnt activity differs between FVB and DUHi, although we cannot orient it as for the BMP pathway. This is consistent with recent findings suggesting that both activation and inhibition of the Wnt pathway is required to make teeth, and Wnt activation needs to be carefully controlled by feedback mechanisms (including a crosstalk with the BMP4 pathway) to enable the sequential formation of teeth (Järvinen et al., 2018).

In conclusion, transcriptomic data further establish a difference in the activation-inhibition balance between the two strains. The balance tends towards weaker BMP4 activity in DUHi mice at the cap transition stage, suggestive of lowered levels of activation in these mice. We next asked if and how these differences could explain an anterior elongation of the adult M1 that is specific to the upper molar.

\section{Lower and upper molar developmental systems consistently differ in their dynamics, regardless the strain}

In a previous study, we had put forward differences between the lower and upper molar developmental system in CD1 mice (Sadier et al., 2019). In both strains, we found similar lower-upper jaw differences as seen in CD1 mice, namely 1) R2 persisted longer in the upper than in the lower jaw, as tested via a Fisher exact test on samples for which data were available for both upper and lower jaws of the same embryo (p=0.04). 2) The M1 Shh expression zone increased in size in upper as in lower jaw, but in the lower jaw only it encompassed the zone of the R2 signaling center (compare Figure 2 Q-T and Supplementary Figure Q-T). Thus, the spatio-temporal dynamics and fate of the R2 signaling center relative to M1 signaling center differs between the two jaws, regardless of difference in activationinhibition mechanisms between strains. This can be considered a conserved developmental property of the lower and upper developmental systems. This may be the foundation for the lower and upper jaw developmental system reacting non-linearly to a same genetic change between FVB and DUHi mice: the increase in R2 signaling center persistence may be all the stronger as the $\mathrm{R} 2$ signaling center is already more persistent in the upper jaw and preserved from an early fusion with the M1 signaling center. But why would this result in anterior elongation in the upper molar only? Answering this question requires that we make 
comparisons between the two jaws in order to reveal how the R2 bud may contribute to the first molar.

\section{The premolar signaling center region contributes to different parts of the lower and upper first molar}

In order to compare developmental relationship between the R2 bud and M1 in the upper and lower jaw of the DUHI and FVB strain, we turned to a genetic trick and traced the fate of R2 signaling center in the developing molar, we turned to a genetic trick. Using a tamoxifeninducible Cre/LacZ line, we were able to induce the marking of Shh-expressing cells and their descendants at different timepoints during tooth development. In practice, cells expressing Shh at the given timepoint of tamoxifen injection (in practice, a time window corresponding to tamoxifen elimination that may be $24-48 \mathrm{~h}$ ) recombine a lacZ transgene, so that these cells and their descendants will then be positive (blue) to a X-gal staining. By inducing the tamoxifen at different timepoints (Figure 5A, B, supplementary table 2 for a summary of all experiments), we could mark cells that descend from either the R2 signaling center and the M1 signaling center population (Figure $5 \mathrm{C}, \mathrm{F}$, treatment at $12.5 \mathrm{dpc}$ ) or from the M1 signaling center population only (Figure 5D-H, treatments at 13.5 and $14.5 \mathrm{dpc}$ ). This was done in a CD1 background, where the dynamics of R2 and M1 signaling centers is well known (Lochovska et al., 2015; Prochazka et al., 2010; Sadier et al., 2019) and molars are morphologically intermediate between FVB and DUHi mice (Figure 1). For upper molars treated during R2 signaling center activity, the anterior part of the tooth is marked at $17.5 \mathrm{dpc}$ (Figure 5C). However, this anterior region is unmarked when tamoxifen is applied later, during M1 signaling center activity only (Figure 5D-E). Thus, the fate of the cell populations of R2 signaling center is the anterior region of the first upper molar. In the case of the first lower molar, the same region is stained in all three conditions (Figure 5F-H), consistent with R2 signaling center being overwritten by the mature M1 signaling center (Sadier et al., 2019). Moreover, the anterior part of the first molar is unstained. Thus, in the lower molar, the R2 signaling center does not contribute specifically to the most anterior part. From these results, we can deduce that a change in R2 size and R2-M1 centers distance, as seen in DUHi upper jaw, will directly elongate the anterior part of the upper M1 (Figure 5C). In contrast, the more modest change seen in the DUHi lower jaw may elongate the M1, but not specifically from its anterior part (compare Figure 5I with Figure 5D-E). We conclude that intrinsic differences between lower and upper M1 developmental systems are responsible for their different 
reactions: marked anterior elongation in the upper molar and a more discrete and isometric elongation of the lower molar (see Figure 5I). 
A

Timing of signalling centre activity
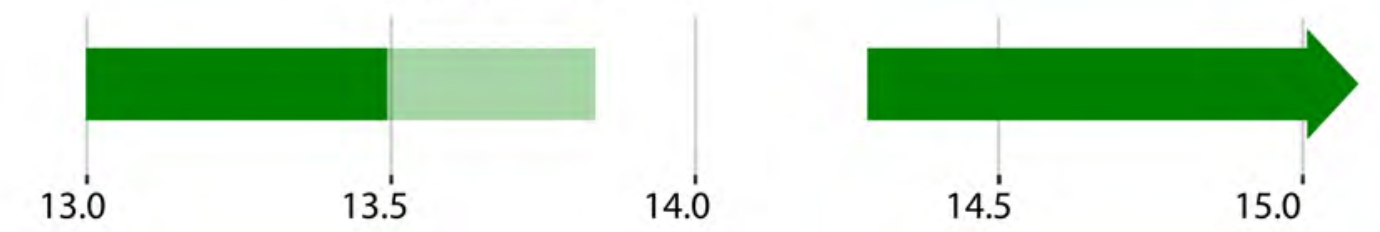

B

Embryo Age (dpc)
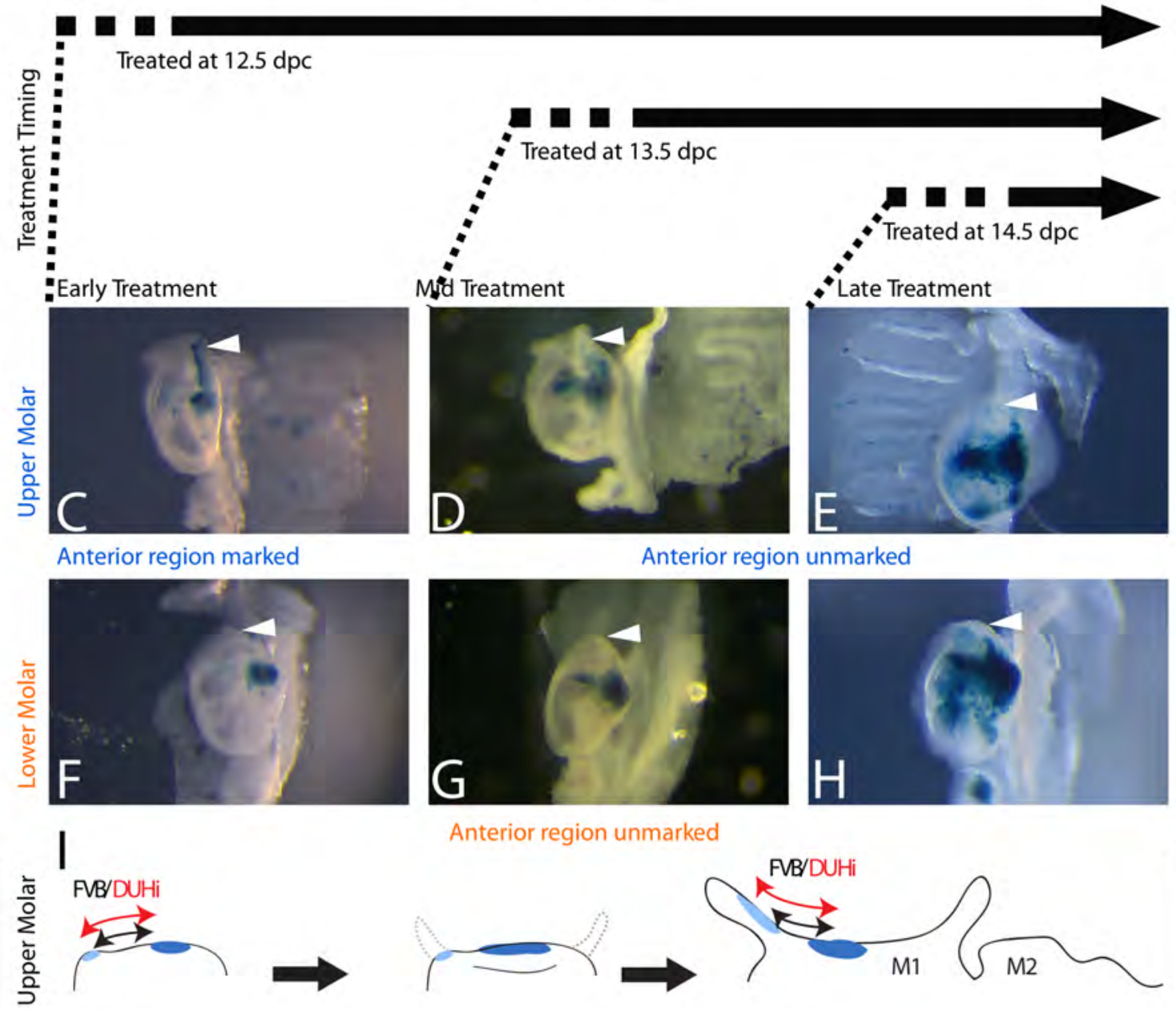

\section{Anterior region unmarked}

FVB/DUHi
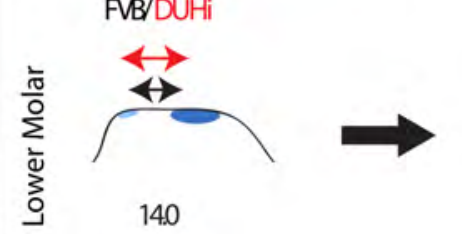

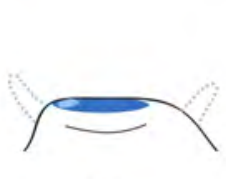

145
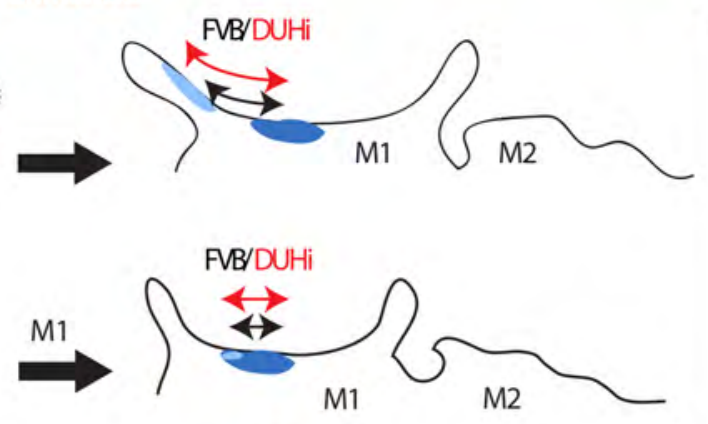

175 


\section{Figure 5: The contribution of $R 2$ and $M 1$ signaling centers to the anterior region of the upper and lower molar}

The timing of R2 and M1 signaling centers activity is shown in green in A. In this context, a tamoxifen-inducible Shh-Cre ${ }^{\mathrm{ERT}}$ line was used to induce the marking of $S h h$-expressing cells from $12.5 \mathrm{dpc}$ (early treatment, time period of treatment corresponds to activity of both R2 and M1 signaling centers), $13.5 \mathrm{dpc}$ or $14.5 \mathrm{dpc}$ (mid and late treatment, time period of treatment corresponds to activity of M1 signaling center only) with $\beta$-galactosidase expression (supplementary table 2). The roughly 12 hours delay for activation is indicated with a dashed line in B. X-gal-stained epithelia of upper (C-E) or lower $(\mathrm{F}-\mathrm{H})$ molars at $17.5 \mathrm{dpc}$ are seen below, with the anterior region of the tooth (white arrowhead) marked in correspondence with the timing of the tamoxifen treatment. The presence of staining in the anterior part of the tooth in $12.5 \mathrm{dpc}$-treated individuals $(\mathrm{C})$ with the lack of staining in later-treated individuals ( $D \& E$ ) indicates that $\mathrm{R} 2$ signaling center contributes to the anterior part of the first upper molar. The scheme in I summarizes results for lineage tracing with induction at 12.5 dpc, marking both R2 signaling center (light blue) and M1 signaling center (dark blue) descendant cells. Following M1 cap transition at $14.5 \mathrm{dpc}$, the tooth will develop anteriorly and posteriorly (shown on the $14.5 \mathrm{dpc}$ scheme with dashed grey line). Only the upper R2 signaling center descendants are involved in anterior cervical loop formation. Differences in size and R2-M1 distance seen between FVB and DUHi strain (black versus red arrows) will preferentially impact the anterior part of the tooth in the upper molar only.

\section{Developmental variation is higher in DUHi than FVB and peaks at the stage of R2-M1 coexistence}

A brief examination revealed that DUHi samples display different states for similar embryonic age (Figure 3 and S7). This suggested that the two strains may exhibit different degree of developmental variability, which we aimed to quantify. This requires disentangling differences in developmental state due to error in embryonic age estimation from real differences due to developmental variation. We developed a method to measure developmental variation, which is described in detail in the Methods section. This method utilises the developmental state scoring system discussed previously. The method works by identifying cases where samples differ greatly in terms of developmental state despite being approximately equally old and allows for statistical comparison of the degree of developmental variation present, via a Wilcoxon rank-sum test. We applied this method to determine whether there is greater developmental variation present in DUHi upper molars than FVB upper molars and in DUHi lower molars than FVB lower molars (Figure 6A and 
Supplementary Figure 10A). Our method yielded a highly significant result in both cases, $(\mathrm{p}<0.001$ for Wilcoxon tests; supplementary text 1 ) where DUHi was more variable than FVB in both cases. Therefore, not only do the developmental trajectories of these two strains differ from one another, but the degree of variation within each strain is not equivalent. Having established that DUHi has more overall developmental variation than FVB, the next step was to develop a method to examine the temporal profile of developmental variation. This was achieved by tracking the difference in developmental state between each embryo and all other embryos of the same strain that were close in age (less than 0.25 days difference in computed embryo age). The local regression line through the developmental state difference present at a given time was then plotted, and is seen in Figure 6B (upper molars) and Supplementary Figure 10B (lower molars). A similar pattern is seen in both upper and lower molars, whereby there is a small peak in developmental variation between computed age $14-15$ cdpc/ weight: $150-250 \mathrm{mg}$, followed by a decrease in developmental variation. This peak is considerably greater, in both duration and magnitude, in DUHi than FVB samples. Some differences exist between the upper and lower molars, however. In the upper molars, it corresponds to the period when two signaling centers coexist and the M1 signaling center expands (14.5-14.75 cdpc / Weight: 200-220mg, Figure 6B). In the lower molar, it also matches this period in DUHi mice (around 14.5 cpdc /Weight: 200mg, Supplementary Figure 10B), but rather corresponds to an earlier variability in termination of R2 signaling for FVB mice (around 14.1 cpdc /Weight: 160mg). 

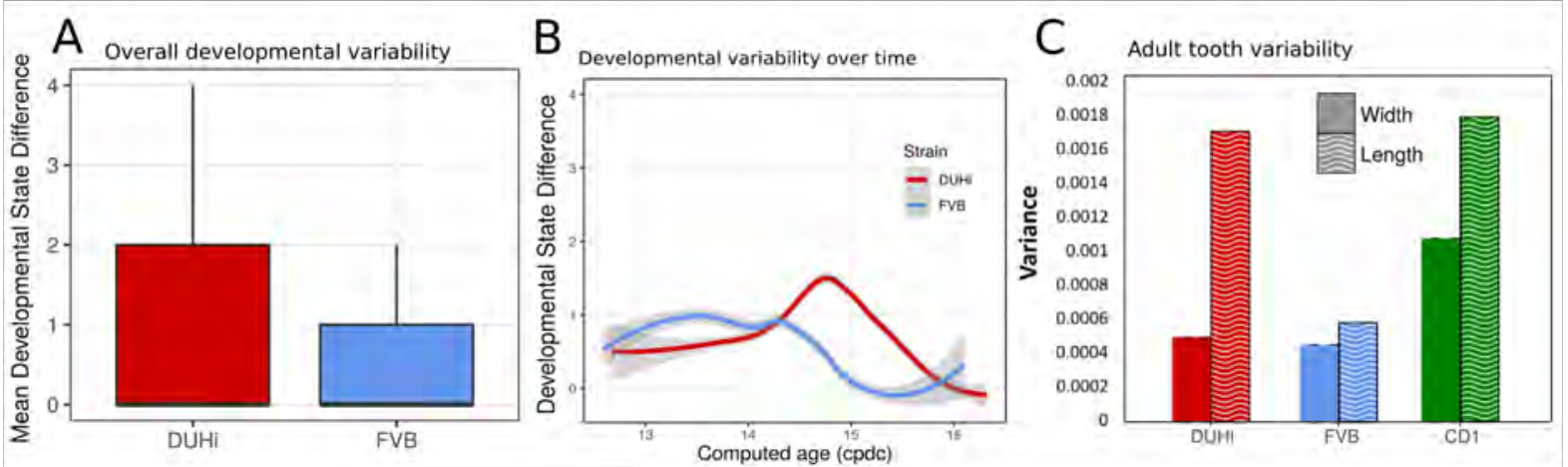

Figure 6: Developmental and adult variation is higher in DUHi first upper molars

(A)-A measure of developmental variation in the developing upper molars of FVB and DUHi strains.

The figure shows a boxplot of developmental state differences calculated for pairs of samples with less than 0,25 difference in computed embryonic age $\left({ }_{c} d p c\right)$. Samples close in age are significantly more different in developmental state in DUHi versus FVB mice, according to a Wilcoxon test $(\mathrm{p}<0.001)$. See the material and methods for further explanation on this measure of developmental variation. (B) The mean developmental state difference between nearby samples (computed embryonic age difference $<0.25$ ) is plotted as the local regression line for both strains (standard deviation shown in grey). (C) Morphological variation in adult first molar, measured as the variance in molar width and length. Variation in length (but not width) is much greater in DUHi than that in FVB (both are inbred strains; $\mathrm{p}=0.095)$, and comparable to the outbred CD1 strain.

\section{The DUHi strain with greater developmental variation has more variable adult molars}

Having established that DUHi embryos display greater variation during development than is seen in their counterparts at equivalent stages in FVB, the next step was to examine whether this developmental variation would be reflected by greater variation in adult morphology. The variation in length and width in DUHi, FVB and CD1 adults was examined for both upper and lower first molars and is shown in Figure 6C and Supplementary Figure 10C. Although they are also an inbred strain, DUHi mice show greater variation in upper molar length than FVB $(\mathrm{p}=0.095)$, to an extent comparable to the outbred CD1 mice. The variation in DUHi upper molar length is consistent with the large degree of variation in R2/M1 development in DUHi embryonic upper jaws. Consistent with the developmental data, this variation in length is specific to the upper molars: In the lower molars, DUHi individuals show little variation in length, albeit large variation in width (Supplementary Figure 10C). 


\section{Discussion}

Evolutionary trajectories are not random, but tend to take preferred routes. Several factors can explain this, among them the ability of developmental systems to vary in particular directions. Then variation typically follows similar trends at different levels, from inter-individual differences to population differences, up to species differences. This underpins the concepts of evolutionary "lines of least resistance" (e.g. (Schluter, 1996)) or "evolutionary predictability" inferred from developmental systems rules (Kavanagh et al., 2007; SalazarCiudad and Jernvall, 2010). Lines of least resistance were primarily thought of as arising from genetic constraints (Schluter, 1996)), whereas the second concept more directly refers to variational properties of developmental systems. Both relate to the old concept of “developmental constraints" (e.g. (Smith et al., 1985)). These concepts are typically tested by matching the variation at different levels (e.g. population/macroevolution) or by simulating it with developmentally realistic models. However, they have rarely been tested by directly examining the variation in developmental systems and its systemic basis, at least for mammalian models. Here, we used a comparative approach, focusing on fine-scale developmental differences that show evolutionary-relevant variation to unravel the generative principles underlying shape variation.

We have used inter-strain variation as a proxy for natural variation. We think this choice is relevant for three reasons: 1) We show that the morphological variation between FVB and DUHi mice recapitulates the morphological variation in the Mus genus and beyond 2) The DUHi mice are a product of artificial selection in the lab for large body size, starting from 6 different mouse strains (Bünger and Herrendörfer, 1994; Bünger et al., 1982). It thus also recapitulates the correlation between anterior elongation and large body size seen in natural populations of Mus musculus domesticus (Renaud et al., 2011). 3) Lab mice were derived from a mix of several mouse subspecies (Mus musculus domesticus, Mus musculus musculus, Mus musculus castaneus). This original substantial amount of genetic variation being now splitted between mouse strains, it is no surprise that different mouse strain could recapitulate trends seen in the Mus genus. 
We show that the anterior development of the first upper molar varies between DUHi and FVB strains. We show inter-related differences such as, in DUHi mice compared to FVB mice, the larger size of $\mathrm{R} 2$ rudimentary bud, the longer persistence of its signaling center, a posteriorisation of the M1 signaling center, a marked tendency for R2 to individualise in culture and differences in gene expression. A similar tendency, although less marked, is seen in the DUHi lower molar. Collectively, these results show that two strains differ in the settings of the activation-inhibition mechanisms patterning the tooth signaling centers. Finding different settings of activation-inhibition mechanisms between two mouse strains is not surprising since mapping studies have found genetic variation segregating with relative molar proportions (Navarro and Murat Maga, 2018). We have indications that the balance is shifted for the BMP4 pathway, with the expression levels of BMP4 targets suggesting overall weaker BMP4 activity in DUHi mice, which would mean reduced activation in DUHi mice. Although R2 partial rescue in a context of reduced activation may appear counter-intuitive at first glance, it is in line with our recent study showing that reduced activation in culture or in the Edar mutant favors the R2 bud in its competition with the M1 signaling center and thereby tends to rescue it (Sadier et al., 2019). We note that the narrow morphology of DUHi (lower and upper) molars as compared with the broad, massive morphology of FVB molars is also consistent with decreased levels of activation in this strain. We have noticed in a study of Orkney house mouse populations that the presence of the anterior cusp was associated with a decrease in overall cusp complexity, again consistent with overall decreased level of activation (Renaud et al., 2018). In conclusion, our results thus suggest that the two lines of least resistance seen in murine rodents: the anterior elongation of the upper molar, and the variation in length/breath seen in both molars are developmentally coupled by a common setting of the activation/inhibition balance.

Our study also sheds light on the developmental reasons why these variations in activationinhibition mechanisms would turn into elongation of the anterior part of the adult molar, specifically and repeatedly in the upper first molar. First, we show that the R2 signaling center is intrinsically stronger in the upper jaw, remains independent of the M1 signaling center and contributes to the anterior part (cervical loop) of upper M1. This is contrasted with the lower jaw, where R2 bud is smaller, included in the mature M1 signaling center (so-called pEK) and R2 signaling center cells do not contribute to the anterior cervical loop, but to a more central part of the molar. As a consequence, subtle variations in activation-inhibition mechanisms that would affect R2 signaling center could specifically impact the anterior part of the upper 
M1 only, whereas this effect could be either buffered in the lower M1 or spread on the whole tooth (DUHi lower M1 are longer than FVB). Secondly, upper molar development appears to be inherently more variable than lower molar development. DUHi and FVB mice are more different in terms of upper molar development than they are in terms of lower molar development; variation in R2 signaling center persistence, in anterior region size and M1 positioning are all stronger for upper than lower molars. This intrinsic variability of the upper molar is also apparent in the phenotypic variability of the DUHi mice. Although these mice are inbred, the upper molar is much variable in length and this is correlated, again, with greater developmental variability in upper R2 signaling center (we note however, that the lower M1 of DUHi mice is highly variable in width, Supplementary Figure 8: this might indicate that the lower M1 also reacts to changes in R2, albeit very differently). This suggests that upper molar development is intrinsically unstable, especially when the tuning of activation-inhibition parameters comes in the "DUHi range". In summary, we provide evidence for developmental particularities acting as the basis of a line of evolutionary least resistance in murine rodents.

A common conception is that developmental variation is minimal at early stages and increases over developmental time. Thus, morphological changes are assumed to result from small changes in development, which result from yet smaller changes in earlier development. This "inverted funnel" bears a certain resemblance to Von Baer's law of embryonic divergence, and to the "hourglass model" of interspecific developmental similarity (Abzhanov, 2013; Irie and Kuratani, 2014). Under this view, mild variations in adult phenotypes should result from almost undetectable or at least late-detectable variation in development. Here, our work identifies strong variation in early tooth development between strains (morphology of the tooth germ and dynamics of signaling centers) in tandem with relatively mild variation in adult phenotype. Besides influencing our view of developmental variation, this has implications for developmental biology practices, especially in the mouse model in which access to embryo is limited for both ethical and cost reasons. For example, heterozygotes are often used as controls for developmental genetic studies, where heterozygotes do not display an obvious phenotype in adults, because it is assumed that the development underlying that phenotype proceeds normally. However, our work here finds greater variation in early development than in the adult phenotype. In systems like that observed here, heterozygotes may have important differences in early development, making their use as controls unreliable. We also note that discrepancies between observations made in different labs will a priori not 
be attributed to a difference in the wild type strain used in each lab. In this context, it is interesting that the recognition of R2 vestigial bud presence in mouse molar development was a matter of debate for several years, and R2 is much less transient and discrete in the CD1 strain, where R2 was first identified, than in FVB, in which much heavier sampling is needed to catch the short developmental window when it is present. Therefore, it is well possible that differences between strains worked to obscure the debate. In conclusion, we believe that enhancing the focus on developmental variation will be important to move on from overly simplistic views of developmental variation that more or less consciously influence our practices in biology. 


\section{References}

Abzhanov, A. (2013). von Baer's law for the ages: lost and found principles of developmental evolution. Trends Genet. 29, 712-722.

Ahn, Y., Sanderson, B.W., Klein, O.D., and Krumlauf, R. (2010). Inhibition of Wnt signaling by Wise (Sostdc1) and negative feedback from Shh controls tooth number and patterning. Development 137, 3221-3231.

Ahnfelt-Rønne, J., Jørgensen, M.C., Hald, J., Madsen, O.D., Serup, P., and HecksherSørensen, J. (2007). An Improved Method for Three-dimensional Reconstruction of Protein Expression Patterns in Intact Mouse and Chicken Embryos and Organs. J. Histochem. Cytochem. 55, 925-930.

Alberch, P., and Gale, E.A. (1985). A DEVELOPMENTAL ANALYSIS OF AN EVOLUTIONARY TREND: DIGITAL REDUCTION IN AMPHIBIANS. Evolution 39, 8 23.

Balic, A., and Thesleff, I. (2015). Tissue Interactions Regulating Tooth Development and Renewal. In Current Topics in Developmental Biology, (Elsevier), pp. 157-186.

Brakefield, P.M. (2006). Evo-devo and constraints on selection. Trends Ecol. Evol. 21, 362368.

Brakefield, P.M. (2011). Evo-devo and accounting for Darwin's endless forms. Philos. Trans. R. Soc. B Biol. Sci. 366, 2069-2075.

Bray, N.L., Pimentel, H., Melsted, P., and Pachter, L. (2016). Near-optimal probabilistic RNA-seq quantification. Nat. Biotechnol. 34, 525.

Bünger, L., and Herrendörfer, G. (1994). Analysis of a long-term selection experiment with an exponential model. J. Anim. Breed. Genet. 111, 1-13.

Bünger, L., Schüler, L., Renne, U., and Kupatz, B. (1982). [Selection and growth of laboratory mice under synchronized estrus conditions. Direct selection success and correlated selection effect on litter size]. Arch. Exp. Veterinarmed. 36, 641-646.

Cho, S.-W., Kwak, S., Woolley, T.E., Lee, M.-J., Kim, E.-J., Baker, R.E., Kim, H.-J., Shin, J.S., Tickle, C., Maini, P.K., et al. (2011). Interactions between Shh, Sostdc1 and Wnt signaling and a new feedback loop for spatial patterning of the teeth. Development 138, 1807-1816.

Echelard, Y., Epstein, D.J., St-Jacques, B., Shen, L., Mohler, J., McMahon, J.A., and McMahon, A.P. (1993). Sonic hedgehog, a member of a family of putative signaling molecules, is implicated in the regulation of CNS polarity. Cell 75, 1417-1430.

Gould, S.J., and Lewontin, R.C. (1979). The Spandrels of San Marco and the Panglossian Paradigm: A Critique of the Adaptationist Programme. Proc. R. Soc. B Biol. Sci. 205, 581598.

Grüneberg, H. (1965). Genes and genotypes affecting the teeth of the mouse. J Embryol Exp Morphol 14, 137-159.

Haara, O., Harjunmaa, E., Lindfors, P.H., Huh, S.-H., Fliniaux, I., Aberg, T., Jernvall, J., Ornitz, D.M., Mikkola, M.L., and Thesleff, I. (2012). Ectodysplasin regulates activatorinhibitor balance in murine tooth development through Fgf20 signaling. Development 139, 3189-3199.

Harjunmaa, E., Kallonen, A., Voutilainen, M., Hämäläinen, K., Mikkola, M.L., and Jernvall, J. (2012). On the difficulty of increasing dental complexity. Nature 483, 324-327.

Harjunmaa, E., Seidel, K., Häkkinen, T., Renvoisé, E., Corfe, I.J., Kallonen, A., Zhang, Z.-Q., Evans, A.R., Mikkola, M.L., Salazar-Ciudad, I., et al. (2014). Replaying evolutionary transitions from the dental fossil record. Nature.

Hayashi, S., and McMahon, A.P. (2002). Efficient Recombination in Diverse Tissues by a Tamoxifen-Inducible Form of Cre: A Tool for Temporally Regulated Gene

Activation/Inactivation in the Mouse. Dev. Biol. 244, 305-318.

Hendrikse, J.L., Parsons, T.E., and Hallgrímsson, B. (2007). Evolvability as the proper focus 
of evolutionary developmental biology. Evol. Dev. 9, 393-401.

Irie, N., and Kuratani, S. (2014). The developmental hourglass model: a predictor of the basic body plan? Development 141, 4649-4655.

Järvinen, E., Shimomura-Kuroki, J., Balic, A., Jussila, M., and Thesleff, I. (2018). Mesenchymal Wnt/ $\beta$-catenin signaling limits tooth number. Development 145, dev158048. Jernvall, J., Kettunen, P., Karavanova, I., MARTIN, L., and Thesleff, I. (1994). Evidence for the role of the enamel knot as a control center in mammalian tooth cusp formation: nondividing cells express growth stimulating Fgf-4 gene. Int. J. Dev. Biol. 38, 463-469.

Kavanagh, K.D., Evans, A.R., and Jernvall, J. (2007). Predicting evolutionary patterns of mammalian teeth from development. Nature 449, 427-432.

Klein, O.D., Minowada, G., Peterkova, R., Kangas, A., Yu, B.D., Lesot, H., Peterka, M., Jernvall, J., and Martin, G.R. (2006). Sprouty Genes Control Diastema Tooth Development via Bidirectional Antagonism of Epithelial-Mesenchymal FGF Signaling. Dev. Cell 11, 181190.

Laland, K., Uller, T., Feldman, M., Sterelny, K., Müller, G.B., Moczek, A., Jablonka, E., Odling-Smee, J., Wray, G.A., Hoekstra, H.E., et al. (2014). Does evolutionary theory need a rethink? Nature 514, 161-164.

Ledevin, R., Chevret, P., Ganem, G., Britton-Davidian, J., Hardouin, E.A., Chapuis, J.-L., Pisanu, B., da Luz Mathias, M., Schlager, S., Auffray, J.-C., et al. (2016). Phylogeny and adaptation shape the teeth of insular mice. Proc. R. Soc. B Biol. Sci. 283, 20152820.

Lesot, H., Peterková, R., Viriot, L., Vonesch, J.L., Turecková, J., Peterka, M., and Ruch, J.V. (1998). Early stages of tooth morphogenesis in mouse analyzed by 3D reconstructions. Eur. J. Oral Sci. 106 Suppl 1, 64-70.

Lochovska, K., Peterkova, R., Pavlikova, Z., and Hovorakova, M. (2015). Sprouty gene dosage influences temporal-spatial dynamics of primary enamel knot formation. BMC Dev. Biol. 15.

Love, M.I., Huber, W., and Anders, S. (2014). Moderated estimation of fold change and dispersion for RNA-seq data with DESeq2. Genome Biol. 15, 550.

Miner, J.H., Patton, B.L., Lentz, S.I., Gilbert, D.J., Snider, W.D., Jenkins, N.A., Copeland, N.G., and Sanes, J.R. (1997). The Laminin $\alpha$ Chains: Expression, Developmental Transitions, and Chromosomal Locations of $\alpha 1-5$, Identification of Heterotrimeric Laminins 8-11, and Cloning of a Novel $\alpha 3$ Isoform. J. Cell Biol. 137, 685-701.

Misonne, X. (1969). African and Indo-australian Muridae. Evolutionary Trends. (Tervuren, Belgique: Musée Royal de l'Afrique Centrale).

Navarro, N., and Murat Maga, A. (2018). Genetic mapping of molar size relations identifies inhibitory locus for third molars in mice. Heredity $121,1-11$.

O’Connell, D.J., Ho, J.W.K., Mammoto, T., Turbe-Doan, A., O’Connell, J.T., Haseley, P.S., Koo, S., Kamiya, N., Ingber, D.E., Park, P.J., et al. (2012). A Wnt-bmp feedback circuit controls intertissue signaling dynamics in tooth organogenesis. Sci. Signal. 5, ra4.

Oster, G.F., Shubin, N., Murray, J.D., and Alberch, P. (1988). EVOLUTION AND

MORPHOGENETIC RULES: THE SHAPE OF THE VERTEBRATE LIMB IN ONTOGENY AND PHYLOGENY. Evolution 42, 862-884.

Pantalacci, S., Sémon, M., Martin, A., Chevret, P., and Laudet, V. (2009). Heterochronic shifts explain variations in a sequentially developing repeated pattern: palatal ridges of muroid rodents. Evol. Dev. 11, 422-433.

Peterka, M., Lesot, H., and Peterková, R. (2002). Body weight in mouse embryos specifies staging of tooth development. Connect. Tissue Res. 43, 186-190.

Peterkova, R., Lesot, H., Vonesch, J.L., Peterka, M., and Ruch, J.V. (1996). Mouse molar morphogenesis revisited by three dimensional reconstruction. I. Analysis of initial stages of the first upper molar development revealed two transient buds. Int. J. Dev. Biol. 40, 1009- 
1016.

Peterková, R., Peterka, M., Viriot, L., and Lesot, H. (2002). Development of the Vestigial Tooth Primordia as Part of Mouse Odontogenesis. Connect. Tissue Res. 43, 120-128. Peterkova, R., Lesot, H., and Peterka, M. (2006). Phylogenetic memory of developing mammalian dentition. J. Exp. Zoolog. B Mol. Dev. Evol. 306B, 234-250.

Peterkova, R., Churava, S., Lesot, H., Rothova, M., Prochazka, J., Peterka, M., and Klein, O.D. (2009). Revitalization of a diastemal tooth primordium in Spry2 null mice results from increased proliferation and decreased apoptosis. J. Exp. Zoolog. B Mol. Dev. Evol. 312B, 292-308.

Peterkova, R., Hovorakova, M., Peterka, M., and Lesot, H. (2014). Three-dimensional analysis of the early development of the dentition. Aust. Dent. J. 59, 55-80.

Prochazka, J., Pantalacci, S., Churava, S., Rothova, M., Lambert, A., Lesot, H., Klein, O., Peterka, M., Laudet, V., and Peterkova, R. (2010). Patterning by heritage in mouse molar row development. Proc. Natl. Acad. Sci. 107, 15497-15502.

Renaud, S., and Auffray, J.-C. (2013). The direction of main phenotypic variance as a channel to evolution: cases in murine rodents. Hystrix Ital. J. Mammal. 24, 85-93.

Renaud, S., Auffray, J.-C., and Michaux, J. (2006). Conserved phenotypic variation patterns, evolution along lines of least resistance, and departure due to selection in fossil rodents.

Evolution 60, 1701-1717.

Renaud, S., Pantalacci, S., Quéré, J.-P., Laudet, V., and Auffray, J.-C. (2009). Developmental constraints revealed by co-variation within and among molar rows in two murine rodents.

Evol. Dev. 11, 590-602.

Renaud, S., Pantalacci, S., and Auffray, J.-C. (2011). Differential Evolvability Along Lines of Least Resistance of Upper and Lower Molars in Island House Mice. PLoS ONE 6, e18951.

Renaud, S., Ledevin, R., Souquet, L., Gomes Rodrigues, H., Ginot, S., Agret, S., Claude, J., Herrel, A., and Hautier, L. (2018). Evolving Teeth Within a Stable Masticatory Apparatus in Orkney Mice. Evol. Biol. 45, 405-424.

Revell, L.J. (2012). phytools: An R package for phylogenetic comparative biology (and other things). Methods Ecol Evol 3, 217-223.

Sadier, A., Twarogowska, M., Steklikova, K., Hayden, L., Lambert, A., Schneider, P., Laudet, V., Hovorakova, M., Calvez, V., and Pantalacci, S. (2019). Modeling Edar expression reveals the hidden dynamics of tooth signaling center patterning. PLOS Biol. 17, e3000064.

Salazar-Ciudad, I., and Jernvall, J. (2010). A computational model of teeth and the developmental origins of morphological variation. Nature 464, 583-586.

Schluter, D. (1996). ADAPTIVE RADIATION ALONG GENETIC LINES OF LEAST RESISTANCE. Evol. Int. J. Org. Evol. 50, 1766-1774.

Sears, K.E. (2014). Quantifying the impact of development on phenotypic variation and evolution: DEVELOPMENT AND PHENOTYPIC VARIATION. J. Exp. Zoolog. B Mol. Dev. Evol. 322, 643-653.

Smith, J.M., Burian, R., Kauffman, S., Alberch, P., Campbell, J., Goodwin, B., Lande, R., Raup, D., and Wolpert, L. (1985). Developmental Constraints and Evolution: A Perspective from the Mountain Lake Conference on Development and Evolution. Q. Rev. Biol. 60, 265287.

Soneson, C., Love, M.I., and Robinson, M.D. (2016). Differential analyses for RNA-seq: transcript-level estimates improve gene-level inferences. F1000Research 4. Viriot, L., Lesot, H., Vonesch, J.L., Ruch, J.-V., Peterka, M., and Peterkova, R. (2002). The presence of rudimentary odontogenic structures in the mouse embryonic mandible requires reinterpretation of developmental control of first lower molar histomorphogenesis. Int. J. Dev. Biol. 44, 233-240.

Wickham, H. (2007). Reshaping Data with the reshape Package. J. Stat. Softw. 21, 1-20. 
Wickham, H. (2009). ggplot2 Elegant Graphics for Data Analysis.

Yokomizo, T., and Dzierzak, E. (2010). Three-dimensional cartography of hematopoietic clusters in the vasculature of whole mouse embryos. Development 137, 3651-3661.

Zerbino, D.R., Achuthan, P., Akanni, W., Amode, M.R., Barrell, D., Bhai, J., Billis, K., Cummins, C., Gall, A., Girón, C.G., et al. (2018). Ensembl 2018. Nucleic Acids Res. 46, D754. 
bioRxiv preprint doi: https://doi.org/10.1101/818484; this version posted October 24,2019 . The copyright holder for this preprint (which

was not certified by peer review) is the author/funder. All rights reserved. No reuse allowed without permission. 


\section{Acknowledgements}

We acknowledge the contribution of the Plateau de Biologie Expérimentale de la Souris (PBES), more especially Marie Teixeira, Farida Henry and Céline Angleraux for DUHi mice recovery and breeding, and the Plateau Technique Imagerie/Microscopie (PLATIM) of SFR Biosciences Gerland-Lyon Sud (UMS344/US8), more especially Claire Lionnet and Christophe Chamot for their support and help with 3D reconstructions. We are grateful to Jeff Miner for the anti-laminin5 antibody and Pascale Chevret for her expertise in the phylogeny of murine rodents. This work was supported by Agence Nationale de la Recherche (ANR-11BSV7-008 "Bigtooth") and Fondation pour la recherche médicale (FRM; SPF20140129165). Salaries were supported by CNRS for SP and SR, Ecole Normale Supérieure de Lyon for MS. This work was supported by the Grant Agency of the Czech Republic (14-37368G) and by the Charles University (Research Program Progress Q29).

\section{Competing interest}

The authors declare no competing interests. 


\section{Supplementary data}

- Supplementary statistical details. Tables for key statistical tests performed for this study.

- Supplementary text 1: RNAseq of DUHi and FVB M1 germs at cap stage: a detailed description of transcriptomic data analysis.

- Supplementary table 1: Scoring criteria used to assess embryonic dental epithelia

- Supplementary table 2: Summary of lineage tracing experiments

- Supplementary Figures 1 to 10

- Supplementary File 1: An excel file for transcriptomic analysis: normalized basemean for all genes with statistical support in our DE analysis, list of DE genes for padj $<0.05$, list of BMP4 pathway target genes extracted from O'Connell et al. and their classification as activated/repressed target of BMP4 pathway, list of BMP4 target genes DE in DUHi/FVB, list of Wnt pathway target genes extracted from O'Connell et al., and their classification as activated/repressed targets; list of Wnt target genes DE in DUHi/FVB.

- Supplementary Methods : Bayesian method to estimate embryonic age for mouse embryos: a detailed description of the method with tables and a figure showing age estimation from the model under 2 different parameter settings, and additional figures comparing results for 3 different age estimations: embryo weight, a " realistic" parameter setting of the model (as in main figures), and a "permissive" parameter setting of the model. 


\section{Supplementary statistical details}

The following tables provide summaries of key statistical tests performed for this study.

\section{Comparing the timing of developmental events between strains}

Logistic regression

$\begin{array}{llllll}\text { Test } & \text { Jaw } & \mathbf{n} & \mathbf{z} & \text { p-value } \\ \text { Strain effect: R2 Shh expression } & \text { Upper } & 182 & -5.857 & 4.71 \mathrm{E}-09 & * * * \\ \text { Strain effect: M1 Shh expression } & \text { Upper } & 182 & 2.668 & 0.007634 & * * \\ \text { Strain effect: Cap transition } & \text { Upper } & 182 & 2.668 & 0.007634 & * * \\ \text { Strain effect: Anterior protrusion } & \text { Upper } & 182 & 1.688 & 0.0914 & \\ & & & & & \\ \text { Strain effect: R2 Shh expression } & \text { Lower } & 192 & -2.207 & 0.0273 & * \\ \text { Strain effect: M1 Shh expression } & \text { Lower } & 192 & 2.593 & 0.00952 & * \\ \text { Strain effect: Cap transition } & \text { Lower } & 192 & 2.593 & 0.00952 & * \\ \text { Strain effect: Anterior protrusion } & \text { Lower } & 192 & 0.21 & 0.8337 & \end{array}$

\section{Comparisons of dental lamina measurements}

t-test

\section{:omparison}

VB vs DUHi: Total dental lamina length

:VB vs DUHi: Pre-M1 spot length

:VB vs DUHi: Post-M1 spot length

:VB vs DUHi: M1 spot length

:VB vs DUHi: Total dental lamina length

:VB vs DUHi: Pre-M1 spot length

:VB vs DUHi: Post-M1 spot length

:VB vs DUHi: M1 spot length

$\begin{array}{lr}\text { Jaw } & \mathbf{n} \\ \text { Upper } & 86 \\ \text { Upper } & 86 \\ \text { Upper } & 86 \\ \text { Upper } & 86 \\ & \\ \text { Lower } & 87 \\ \text { Lower } & 87 \\ \text { Lower } & 87 \\ \text { Lower } & 87\end{array}$

$\begin{array}{rrr}\text { DUHi mean } & \text { FVB mean } & \text { p-value } \\ 594.1256 & 572.0826 & 0.238 \\ 159.1757 & 126.1684 & 0.003^{\text {** }} \\ 184.2302 & 212.646 & 0.009^{* *} \\ 220.47 & 237.257 & 0.1851 \\ & & \\ 502.8327 & 496.7276 & 0.758 \\ 144.3497 & 152.5548 & 0.3185 \\ 214.2619 & 216.1992 & 0.9109 \\ 195.4378 & 180.8119 & 0.2614\end{array}$

\section{Comparison of outcomes of culture between strains}

Fisher's exact test

$\begin{array}{llrr}\text { Comparison } & \mathbf{n} & \text { p-value } \\ \text { DUHi vs FVB: separated vs incorporated } & & 36 & 0.015\end{array}$ *

\section{Comparisons of proportions of up/downregulated genes}

Chi-squared tests

\section{Comparison}

Bmp: up/downregulation in DUHi vs FVB

Wnt: up/downregulation in DUHi vs FVB $\mathbf{n}$

27
43

$\chi^{2}$

6.259

0.209 p-value

0.0124

0.6473 


\section{Comparing rarity of $\mathbf{R} 2$ spot between upper and lower jaws}

Cross-tabulation (lower and upper sample from the same embryo)

\begin{tabular}{l|rr} 
& Upper R2 absent & Upper R2 present \\
Lower R2 absent & 76 & 21 \\
Lower R2 present & 8 & 24
\end{tabular}

Fisher's exact test

\begin{tabular}{llrrr|}
\hline Comparison & $\mathbf{n}$ & \multicolumn{1}{c|}{ p-value } \\
Difference in rarity of R2 spot & & 129 & 0.04
\end{tabular}$*$

\section{Overall variability of developmental trajectories}

Wilcoxon rank-sum test

\section{Comparison}

FVB vs DUHi: overall developmental variation FVB vs DUHi: overall developmental variation
Jaw

Upper Lower n

2616

1802

\section{p-value}

4.82E-09

$1.09 \mathrm{E}-17$

\section{Comparisons of variation in adult molar length/width}

test for equality of two variances between two groups, performed in Systat.

\begin{tabular}{|l|l|l|l|}
\hline comparison & jaw & n & p-value \\
\hline $\begin{array}{l}\text { DUHi/FVB length } \\
\text { variation }\end{array}$ & Upper & 30 & 0.095 \\
\hline $\begin{array}{l}\text { DUHi/FVB width } \\
\text { variation }\end{array}$ & Upper & 30 & 0.996 \\
\hline $\begin{array}{l}\text { DUHi/FVB length } \\
\text { variation }\end{array}$ & Lower & 30 & 0.710 \\
\hline $\begin{array}{l}\text { DUHi/FVB width } \\
\text { variation }\end{array}$ & Lower & 30 & 0.111 \\
\hline
\end{tabular}




\section{Supplementary Text 1: RNAseq of DUHi and FVB M1 germs at cap stage}

For each strain, we sampled 3 embryos with similar body weight (DUHi: 196, 219 and 239 $\mathrm{mg})$ and FVB $(195,215$ and $233 \mathrm{mg})$ for which we obtained both lower and upper tooth germ samples. The morphology of the tooth germs during the dissection process indicated that only the oldest DUHi embryo had just accomplished cap transition, while all three FVB embryos had just accomplished cap transition.

We retrieved 3619 DE (Differentially Expressed) genes (6 samples in FVB / in DUHi, jaw treated as a factor in DESeq2: jaw + Strain). This high number of genes was partly due to the slight developmental time difference between FVB and DUHi samples around cap transition, with DUHi samples tending to be slightly younger than FVB samples (e.g. FVB-DUHi genes partly overlapped with genes differentially expressed across the 3 replicates ordered in time by embryo weight). In particular, enamel knot genes (e.g. Shh, Dkk4, Slit1) appeared upregulated in FVB samples that have just undergone cap transition, as compared with the youngest DUHi samples. Genes with GO terms (searched with Gorilla tool) associated with mitosis (mitotic cell cycle process, $\mathrm{p}$-value $1.3710^{-11}$; chromosome segregation $1.74 .10^{-7}$ ) were enriched in FVB samples, whereas DUHi samples were enriched for amino-acid and carbohydrate metabolism genes (rRNA metabolic process p-value 4.76. 10 $0^{-6}$, organonitrogen compound biosynthetic process p-value 9.82. 10 $0^{-6}$ ) suggesting that FVB samples were enriched in mitotic cells whereas DUHi samples are enriched in $\mathrm{G} 1 / \mathrm{S} / \mathrm{G} 2$ growing cells. This might reflect the slight developmental delay in DUHi samples, if cap transition is associated with a sudden burst of mitosis. Another possibility is that this reflects metabolic differences between the two strains, since DUHi mice are large-sized mice.

First, we checked genes known to be involved in the formation of supernumerary tooth anterior to M1, resembling the premolar lost during mouse evolution. Among DE genes, we found two genes (Spry2, Sostdc1) whose mutation rescues premolar formation (Sup Figure 8). Spry4, Rsk2, Gas 1, Lrp4, Eda, Edar, Fgf20, whose knock-out or overexpression also rescues premolar formation were not differentially expressed. However, in differential expression analyses conducted independently on lower and upper jaw, Gas 1 was DE in lower jaw (but unbiased in upper jaw) and $E d a$ was marginally significantly DE in the upper jaw only). Finally, treatments interfering with $S h h$ also rescue premolar formation (Cho et al., 2011; Harjunmaa et al., 2012). We note that several Shh pathway genes are downregulated in DUHi mice (e.g. Shh, Gli1, Ptch1). However, because Shh is rapidly and strongly upregulated at cap 
transition ((Prochazka et al., 2010); this is also visible in our transcriptomes, see the supplementary table), it is hard to disentangle a true downregulation of the $S h h$ pathway from an artifact caused by the developmental delay observed in DUHi mice.

Next, we examined the BMP4 and Wnt pathways in detail, based on a list of regulatory relationships published by O'Connell et al. 2012. This list contains a number of presumed targets activated or repressed by each pathway, based on downregulation and upregulation in mutants or upon treatments (e.g. treatment with BMP4, treatment against GSK3 to activate the Wnt pathway). We removed ambiguous genes from this list (i.e. that were described as activated or repressed depending on the tissue examined, epithelium or mesenchyme; e.g. dlx1). Then for each pathway, we examined genes of this list that were also differentially expressed between FVB and DUHi strains. Because the activation-inhibition (A-I) balance involves many genes with complex regulatory feedbacks within and between these two key pathways (and other pathways), we did not expect a change in the A-I balance to shift all targets genes in a consistent direction. Rather, we expected to find a different equilibrium, with genes changed in both directions, but that may nevertheless collectively indicate greater or instead weaker activation of these pathways in the DUHi mice. Genes were considered to be indicators of greater pathway activity in DUHi as compared to FVB, if they were both activated targets in the regulatory list and upregulated in DUHi strain, or both repressed target genes and downregulated in DUHi strain. Reciprocally, genes were considered to be indicators of weaker pathway activity in DUHi if they were both activated targets in the regulatory list and downregulated in DUHi strain, or both repressed target genes and upregulated in DUHi strain. As mentioned in the main text, we found a marked bias for indicators of weaker BMP4 activity in DUHi samples. Although the low expression levels of some activated BMP4 targets in DUHi may partially be attributed to the developmental delay between strains (ie. BMP4 targets that are upregulated at the cap transition could have lower expression levels in the youngest DUHi samples because they have not yet accomplished the cap transition), repressed BMP4 targets exhibited high expression levels in DUHi without any obvious correlation with time differences in samples. Therefore, we believe that the low BMP4 activity in DUHi samples is not artefactually driven by the temporal difference in sampling. 


\section{Supplementary Table 1: Scoring criteria used to assess embryonic dental epithelia}

\begin{tabular}{lll} 
Criterion & Severity & Phenotype \\
\hline R2 Shh expression & 0 & no expression \\
\hline & 1 & weak expression in a small zone \\
\hline M1 Shh expression & 0 & no expression \\
\hline & 1 & early signaling center: strong expression in a \\
& circular zone \\
\hline & 2 & mature signaling center: strong expression in a \\
& large oval zone \\
\hline Cap transition & 0 & width constant along length of dental epithelium \\
\hline & 1 & slight widening of dental epithelium near the \\
& signaling center \\
\hline Anterior protrusion & 0 & cap transition completed \\
\hline & 1 & conspicuous protuberance in R2 zone \\
\hline
\end{tabular}

\section{Supplementary table 2: summary of lineage tracing experiments}

Note: All specimens after tamoxifen administration at 12.5, 13.5, $14.5 \mathrm{dpc}$ respectively were harvested at $17.5 \mathrm{dpc}$.

\begin{tabular}{|c|c|c|c|}
\hline $\begin{array}{l}\text { Tamoxifen } \\
\text { administration }\end{array}$ & Injection order & $\begin{array}{l}\text { Number of positive } \\
\text { specimens in the } \\
\text { distinct Cre } \\
\text { activation }\end{array}$ & $\begin{array}{l}\text { Method of } \\
\text { visualization }\end{array}$ \\
\hline \multirow[t]{5}{*}{12.5} & 1. & 4 & $\begin{array}{l}\text { Whole mount X- } \\
\text { gal staining }\end{array}$ \\
\hline & 2. & 1 & $\begin{array}{l}\text { Dissociation of } \\
\text { epithelia }\end{array}$ \\
\hline & 3. & 3 & $\begin{array}{l}\text { Dissociation of } \\
\text { epithelia }\end{array}$ \\
\hline & 4. & 2 & $\begin{array}{l}\text { Dissociation of } \\
\text { epithelia }\end{array}$ \\
\hline & TOTAL & 10 & \\
\hline
\end{tabular}




\begin{tabular}{|c|c|c|c|}
\hline \multirow[t]{6}{*}{13.5} & 1. & 2 & $\begin{array}{l}\text { Whole mount X- } \\
\text { gal staining }\end{array}$ \\
\hline & 2. & 3 & $\begin{array}{l}\text { Whole mount X- } \\
\text { gal staining }\end{array}$ \\
\hline & 3. & 2 & $\begin{array}{l}\text { Dissociation of } \\
\text { epithelia }\end{array}$ \\
\hline & 4. & 1 & $\begin{array}{l}\text { Dissociation of } \\
\text { epithelia }\end{array}$ \\
\hline & 5. & 2 & $\begin{array}{l}\text { Dissociation of } \\
\text { epithelia }\end{array}$ \\
\hline & TOTAL & 10 & \\
\hline \multirow[t]{5}{*}{14.5} & 1. & 2 & $\begin{array}{l}\text { Whole mount X- } \\
\text { gal staining }\end{array}$ \\
\hline & 2. & 1 & $\begin{array}{l}\text { Dissociation of } \\
\text { epithelia }\end{array}$ \\
\hline & 3. & 2 & $\begin{array}{l}\text { Dissociation of } \\
\text { epithelia }\end{array}$ \\
\hline & 4. & 4 & $\begin{array}{l}\text { Dissociation of } \\
\text { epithelia }\end{array}$ \\
\hline & TOTAL & 9 & \\
\hline
\end{tabular}

\section{Supplementary figures}



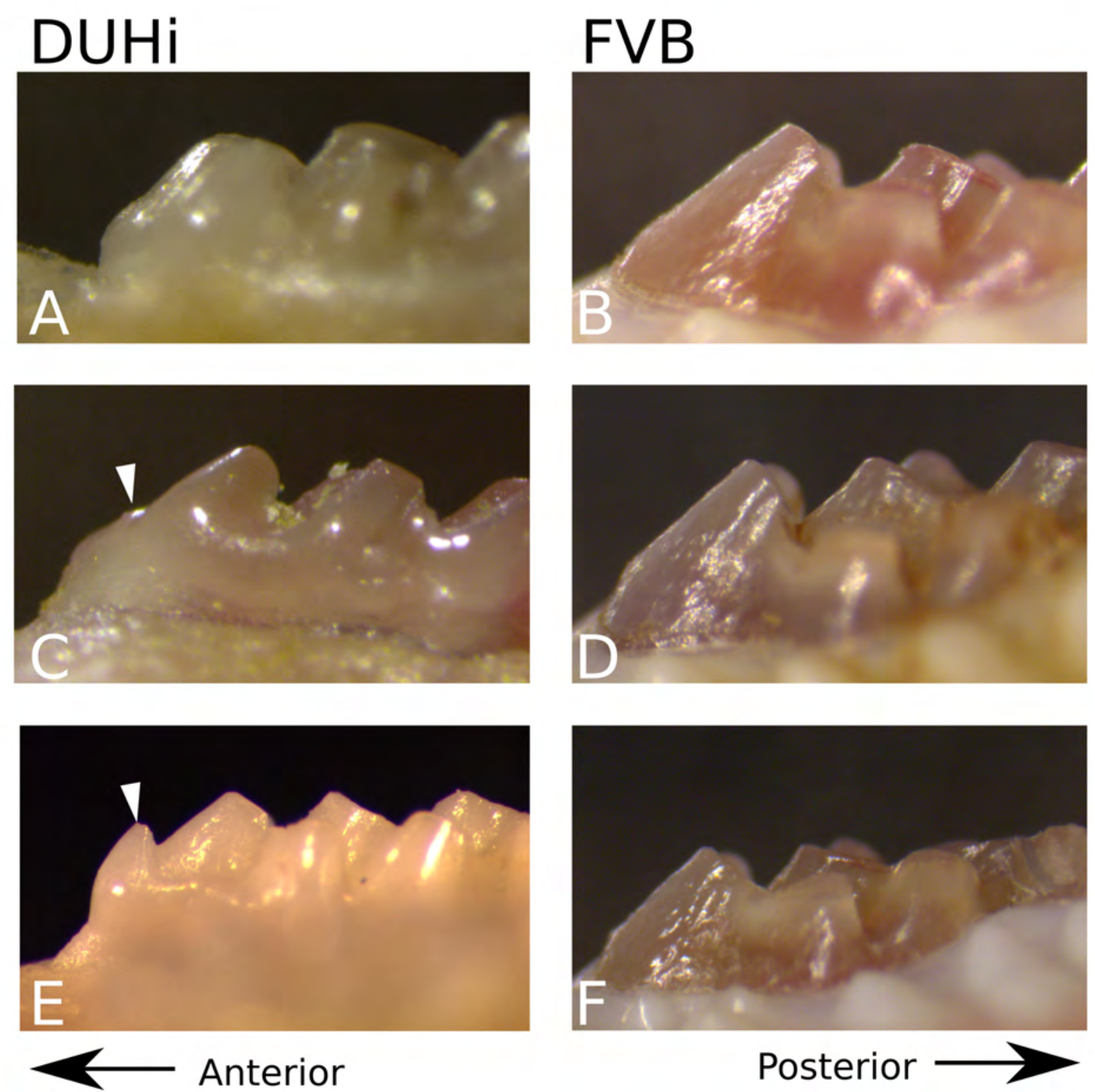

\section{Supplementary Figure 1: Representative examples of adult upper molar morphology in}

\section{DUHi and FVB}

Side views of tooth morphology in DUHi (A, C, E) and FVB (B, D, F) mouse strains. Three individuals are shown for each strain, displaying the variation of the morphology present. The anterior part of the molar is to the left. Where present or partially present, the additional anterior small cusp is marked with a white arrow. 
DUHi lower molars $13.9 \mathrm{cdpc}$

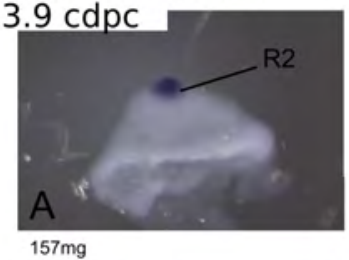

$14.25 \mathrm{cdpc}$

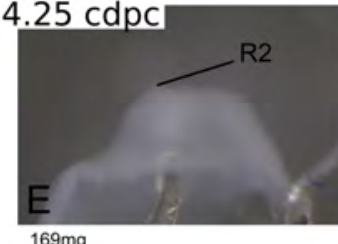

$14.5 \mathrm{cdpc}$
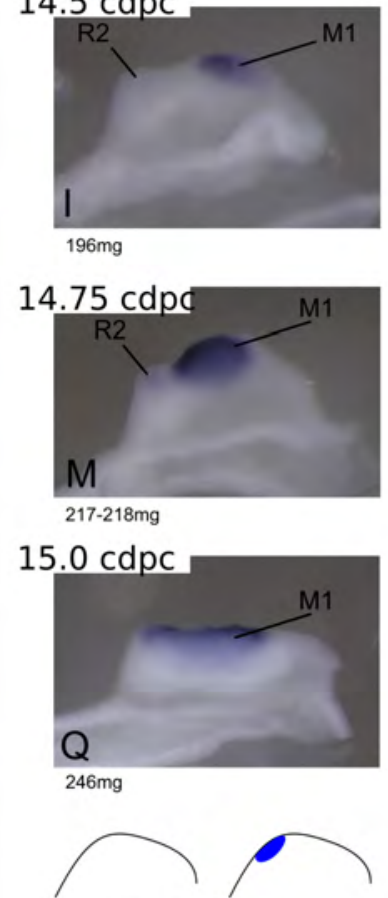

DUHi series

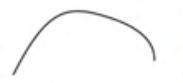

FVB series

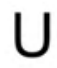

U
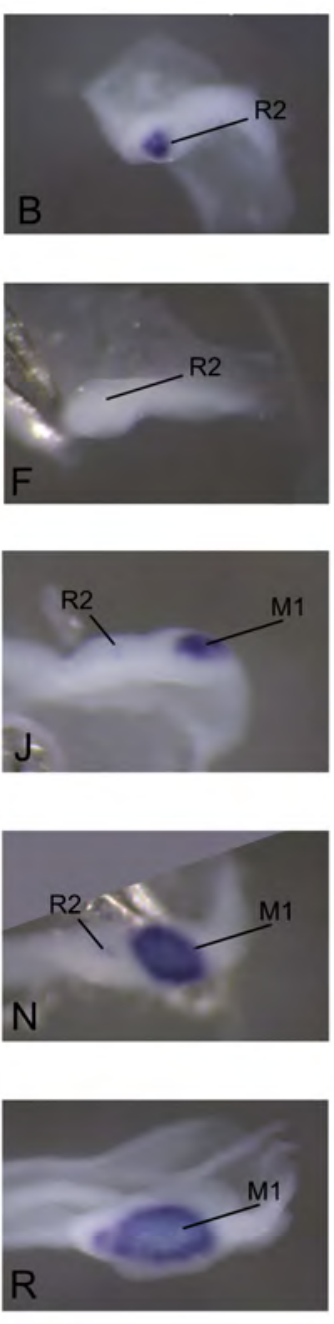

FVB lower molars
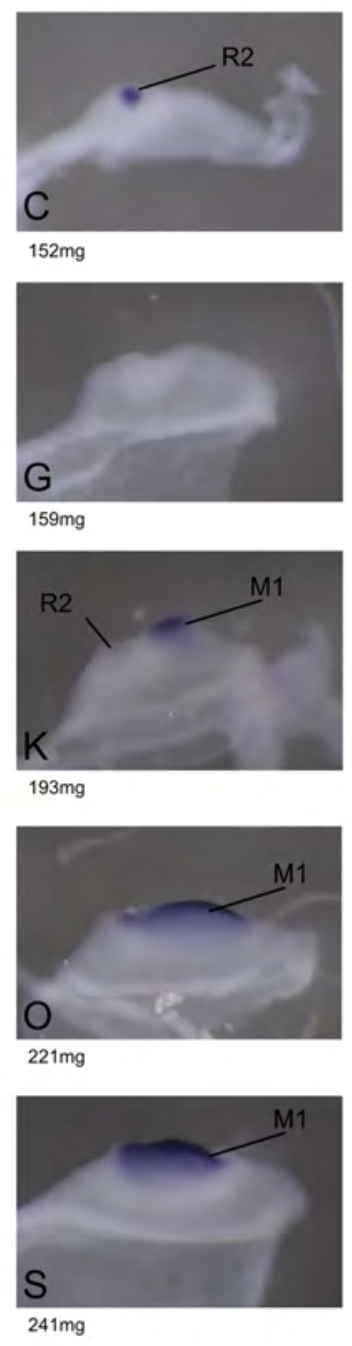

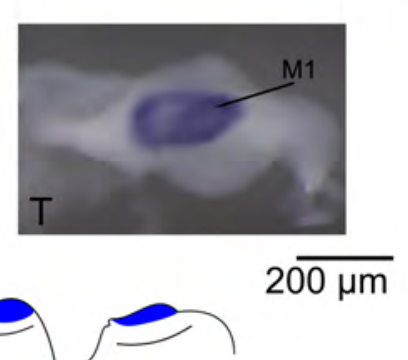

$20 \overline{\mu m}$
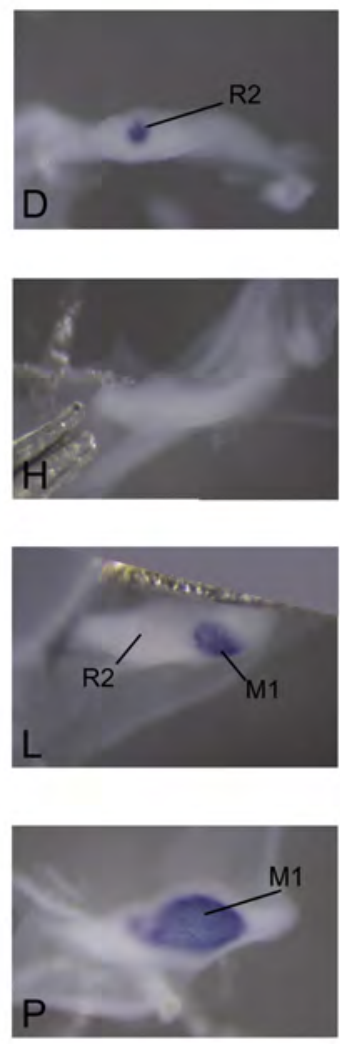
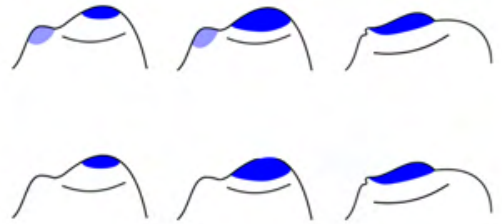

Anterior

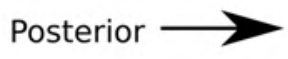

Supplementary Figure 2: Comparative early molar tooth development in lower molar epithelia of DUHi and FVB mice

Dissociated lower dental epithelium epithelia of DUHi (left most two columns) and FVB embryos (right-most two columns), marked for Shh expression with in situ hybridisation. Samples represent a developmental series of early molar development, corresponding to $12.5 \mathrm{dpc}$ to $15.0 \mathrm{dpc}$ in FVB. Embryo weight is noted below each sample and its equivalent in computed embryonic age is noted above each row of samples. Two images of each sample, a 
bioRxiv preprint doi: https://doi.org/10.1101/818484; this version posted October 24,2019 . The copyright holder for this preprint (which

was not certified by peer review) is the author/funder. All rights reserved. No reuse allowed without permission.

side and a top view, are shown. Scale bar $=200 \mu \mathrm{m}$. Developmental progression is summarized in schematic form below (U). 

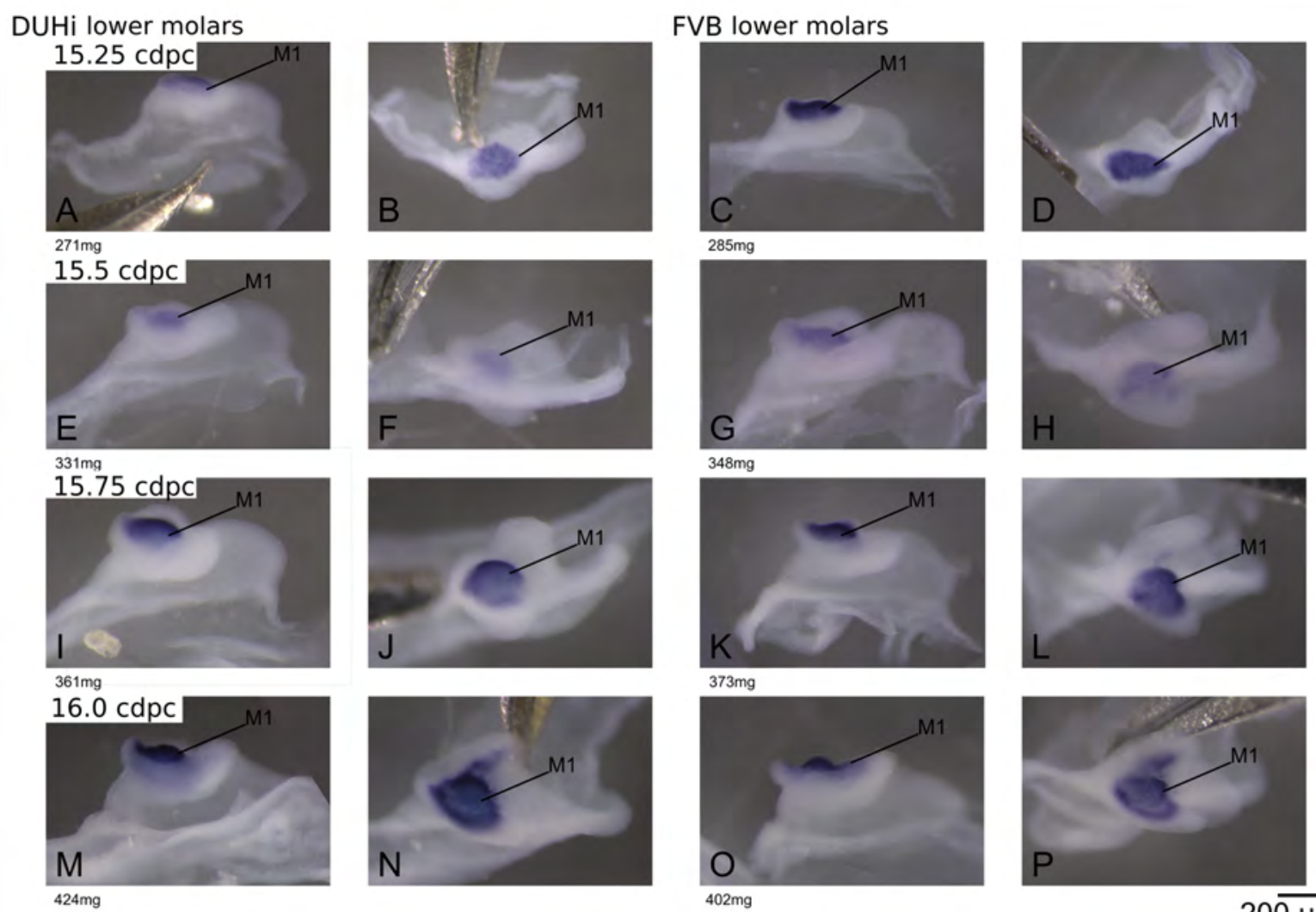

$348 \mathrm{mg}$
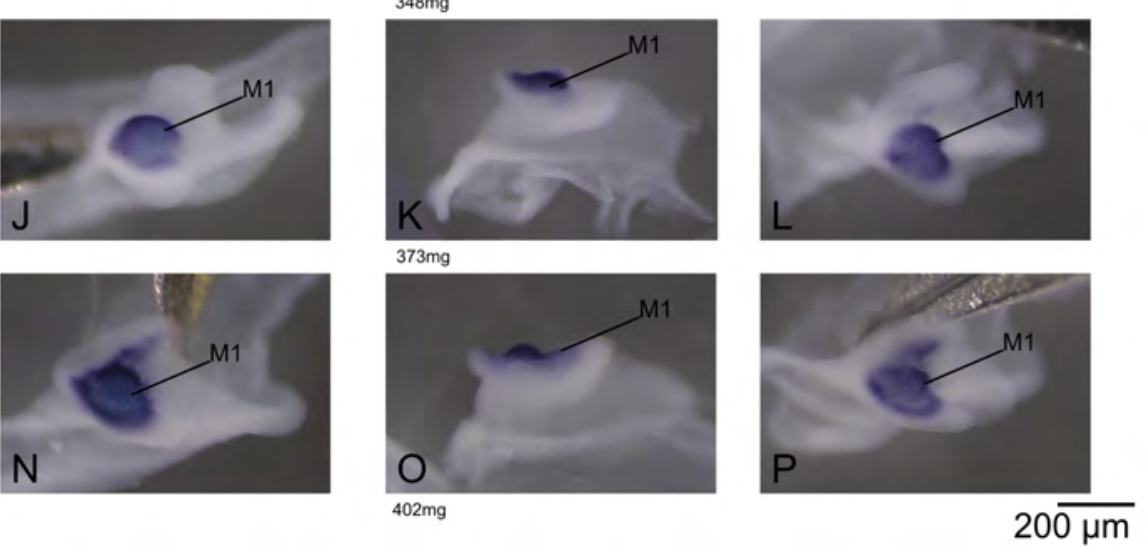

Supplementary Figure 3: Comparative late molar tooth development in lower molar epithelia of DUHi and FVB mice

Dissociated lower dental epithelium epithelia of DUHi (left most two columns) and FVB embryos (right-most two columns), marked for Shh expression with in situ hybridisation. Samples represent a developmental series of later molar development, roughly corresponding to $15.0 \mathrm{dpc}$ to $16.5 \mathrm{dpc}$ in FVB. Embryo weight is noted below each sample and its equivalent in computed embryonic age is noted above each row of samples. Two images of each sample, a side and a top view, are shown. Scale bar $=200 \mu \mathrm{m}$. The dental epithelium is oriented with anterior part to the left. 
DUHi upper molars

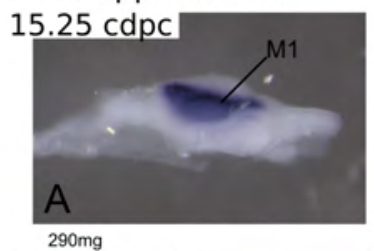

$15.5 \mathrm{cdpc}$

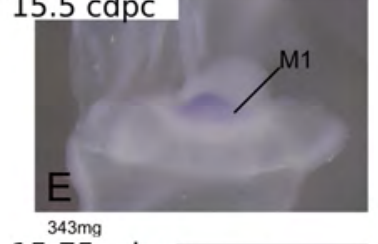

$15.75 \mathrm{cdpc}$
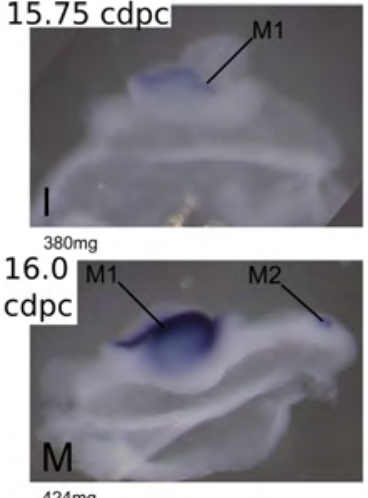
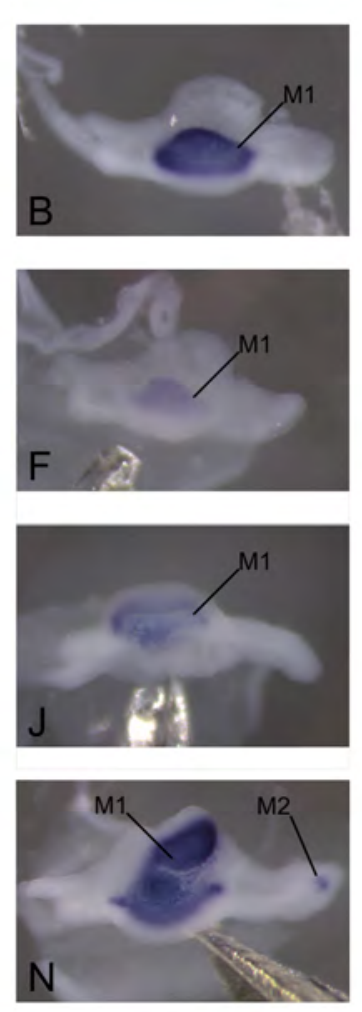

FVB upper molars
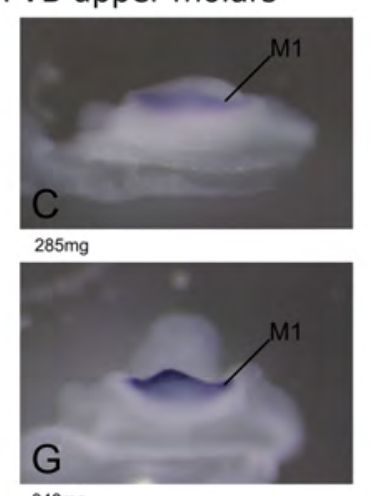

$348 \mathrm{mg}$
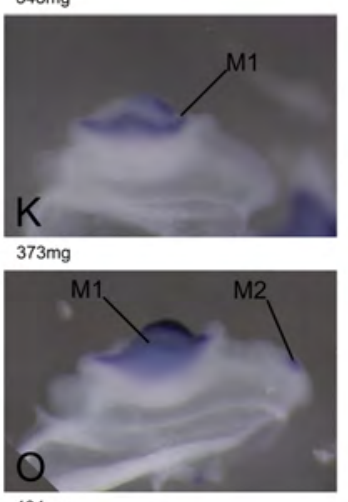

$404 \mathrm{mg}$
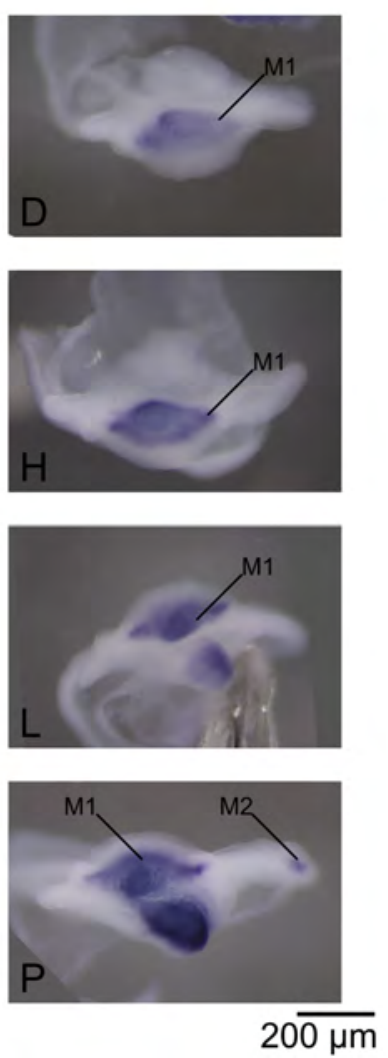

Supplementary Figure 4: Comparative late molar tooth development in upper molar epithelia of DUHi and FVB mice

Dissociated upper dental epithelium epithelia of DUHi (left most two columns) and FVB (right-most two columns) embryos, marked for Shh expression with in situ hybridisation.

Samples represent a developmental series of later molar development, roughly corresponding to $15.0 \mathrm{dpc}$ to $16.5 \mathrm{dpc}$ in FVB. Embryo weight is noted below each sample and its equivalent in computed embryonic age is noted above each row of samples. Two images of each sample, a side and a top view, are shown. Scale bar $=200 \mu \mathrm{m}$. The dental epithelium is oriented with anterior part to the left. 


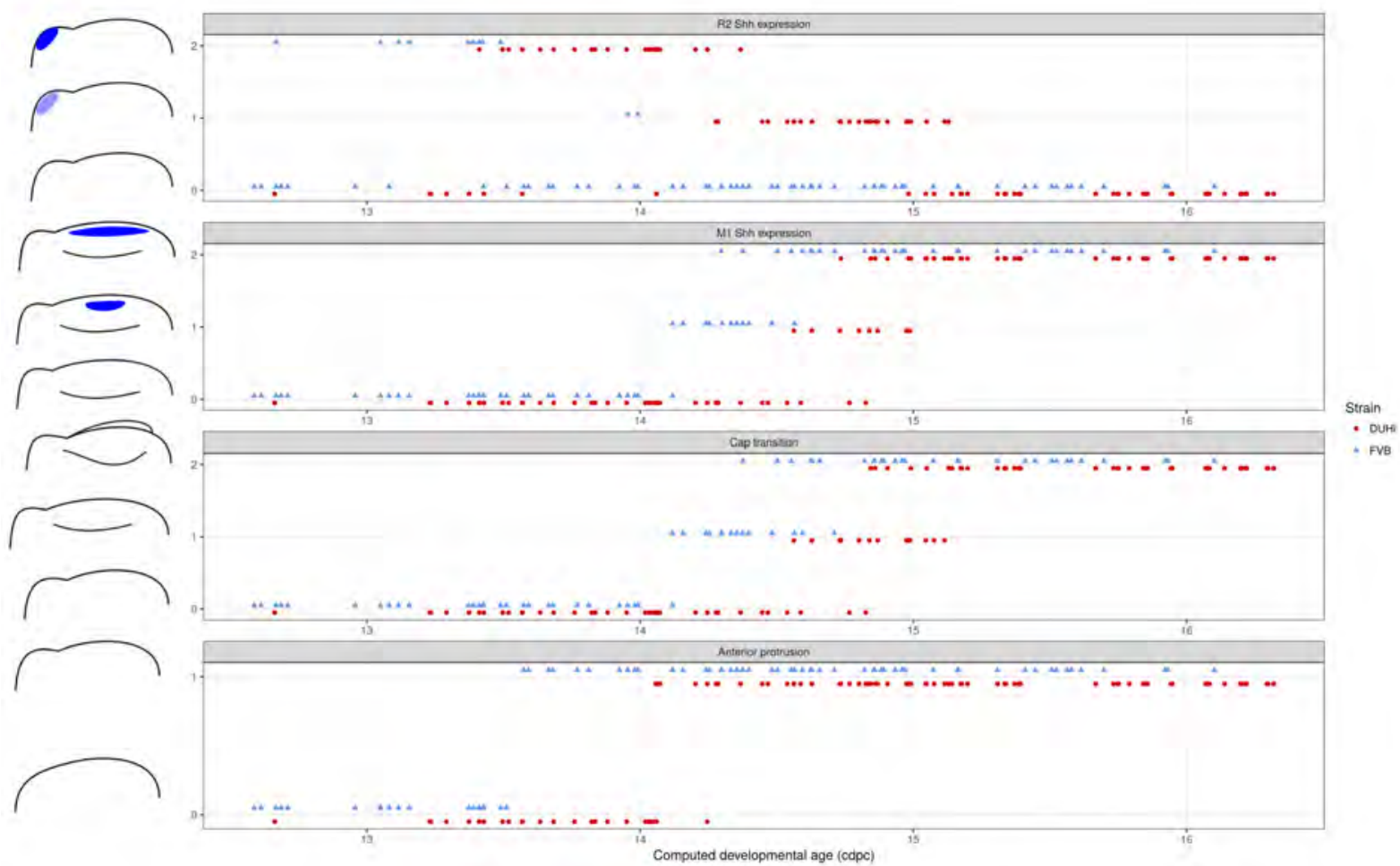

Supplementary Figure 5: Comparative progression of scored characteristics in upper dental epithelium

Progression of four characters (R2 Shh expression, M1 Shh expression, cap transition and anterior protrusion of the dental epithelium) is depicted in samples of upper molars from two strains (FVB and DUHi). The temporal axis is provided by computed embryonic age ( $\mathrm{d} d \mathrm{pc}$ ). All samples were scored using the criteria provided in Supplementary Table 1. 


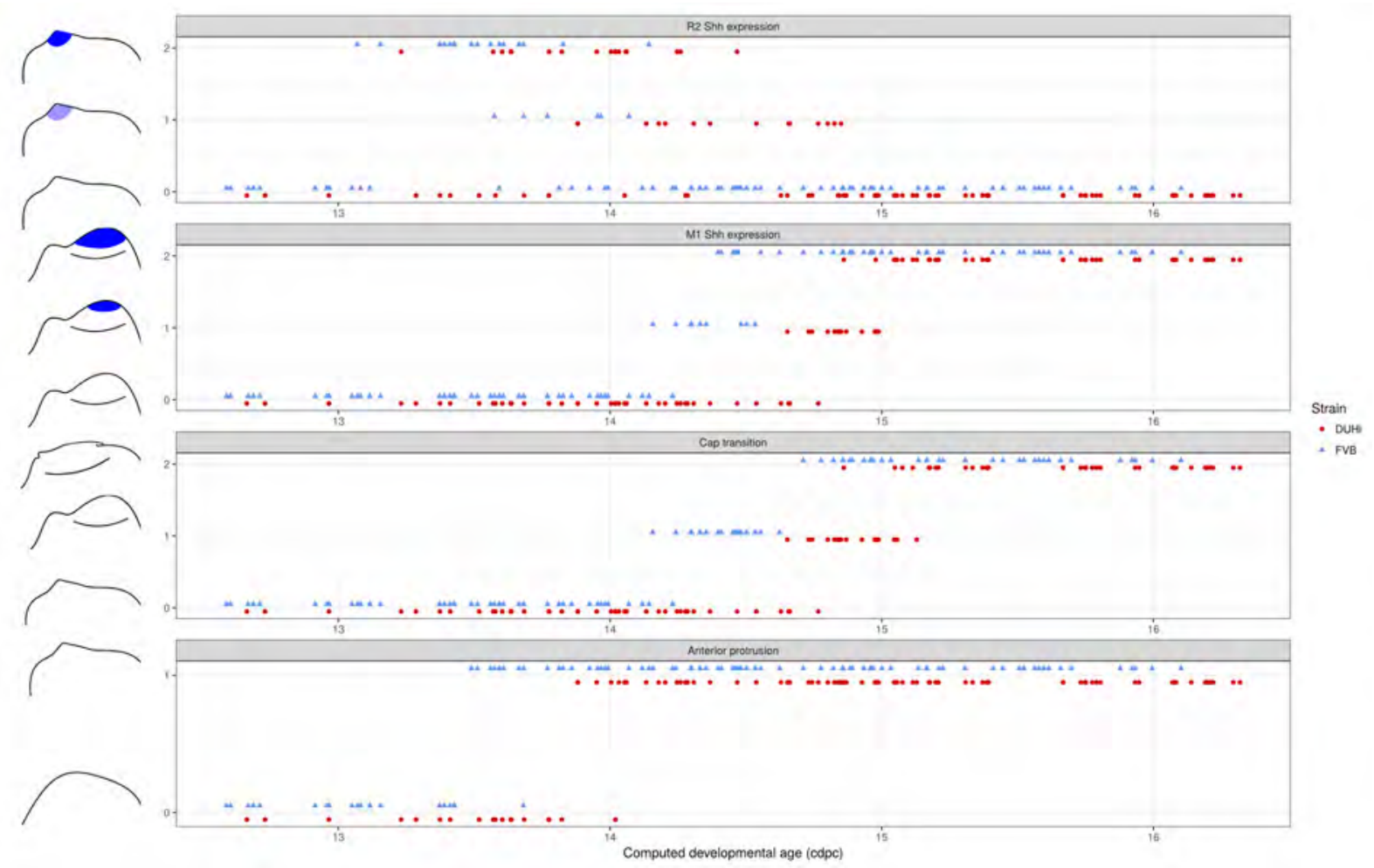

Supplementary Figure 6: Comparative progression of scored characteristics in lower dental epithelium

Progression of four characters (R2 Shh expression, M1 Shh expression, cap transition and anterior protrusion of the dental epithelium) is depicted in samples of lower molars from two strains (FVB and DUHi). The temporal axis is provided by computed embryonic age ( $\mathrm{dpc}$ ). All samples were scored using the criteria provided in Supplementary Table 1. 


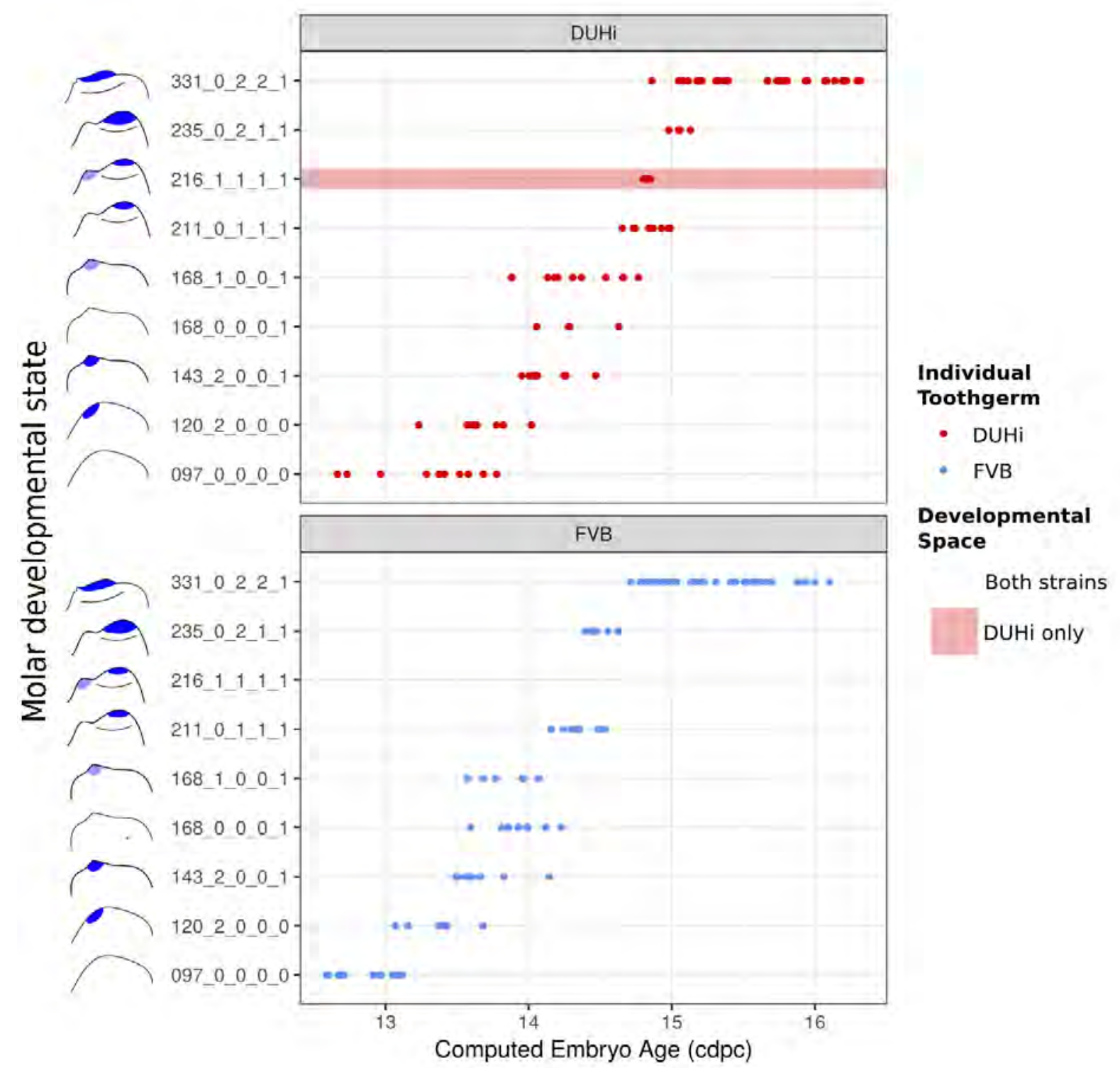

Supplementary Figure 7: The range of possible developmental states differs between FVB and DUHi developing lower molars

Temporal distribution of developmental state of the developing lower molar, produced by combining a value for each of the four scores for a given sample, based on criteria from Supplementary Table 1. Each of the developmental states observed are shown and ordered according to the average embryonic weight of the samples within that group. Exclusive 
developmental states are highlighted according to whether they are found in DUHi only (red) or in FVB only (blue). The temporal axis is given by computed embryonic age (cdpc).
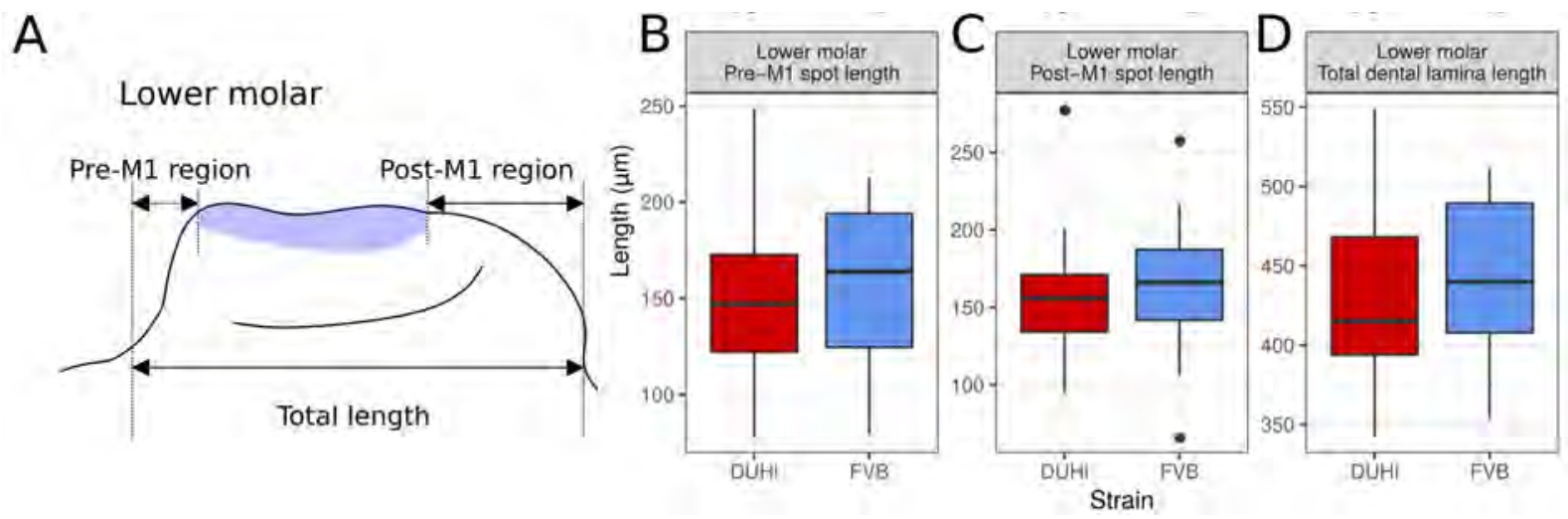

\section{Supplementary Figure 8: comparative measurements in lower molar dental epithelium}

Panel A shows the three measurements taken from all epithelial samples of a computed age of 14.2515 cpdc (between 180 and 250mg weight), comparing DUHi with FVB samples. Boxplots B-D show the results for three of them in lower molars. The measurements were taken between the anterior or posterior limit of dental epithelium and anterior or posterior limit of the M1 signaling center, respectively. Note that the pre-M1 signaling center region tends to be longer in FVB (not significant). This is due to a smoother slope of the anterior epithelium: compare $\mathrm{E}$ with $\mathrm{G}$, I with $\mathrm{K}, \mathrm{M}$ with $\mathrm{O}$ and Q with S, on Supplementary Figure 2. Taken together with the fact that in the lower jaw, R2 signaling center is integrated in the M1 signaling center, this measure is poorly informative on R2 bud size in the lower jaw, in contrast with the upper jaw. 
A

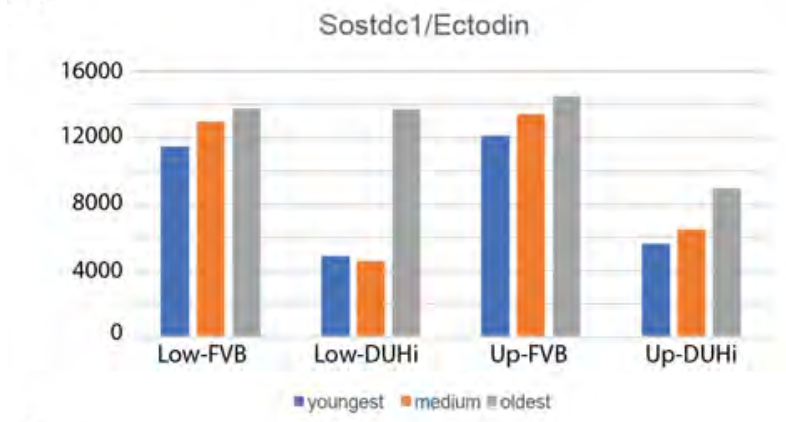

B

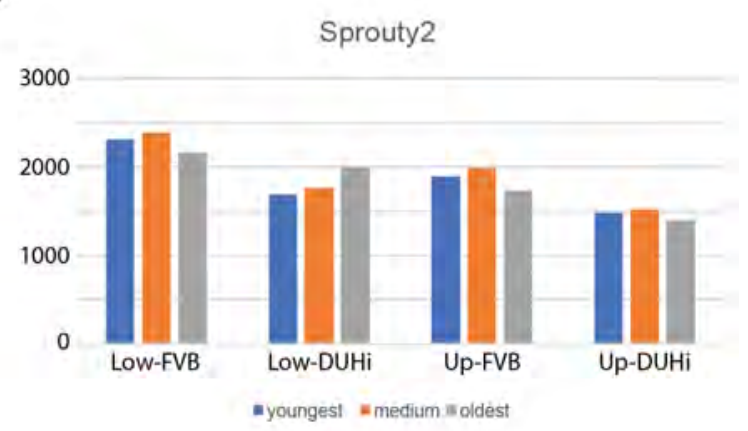

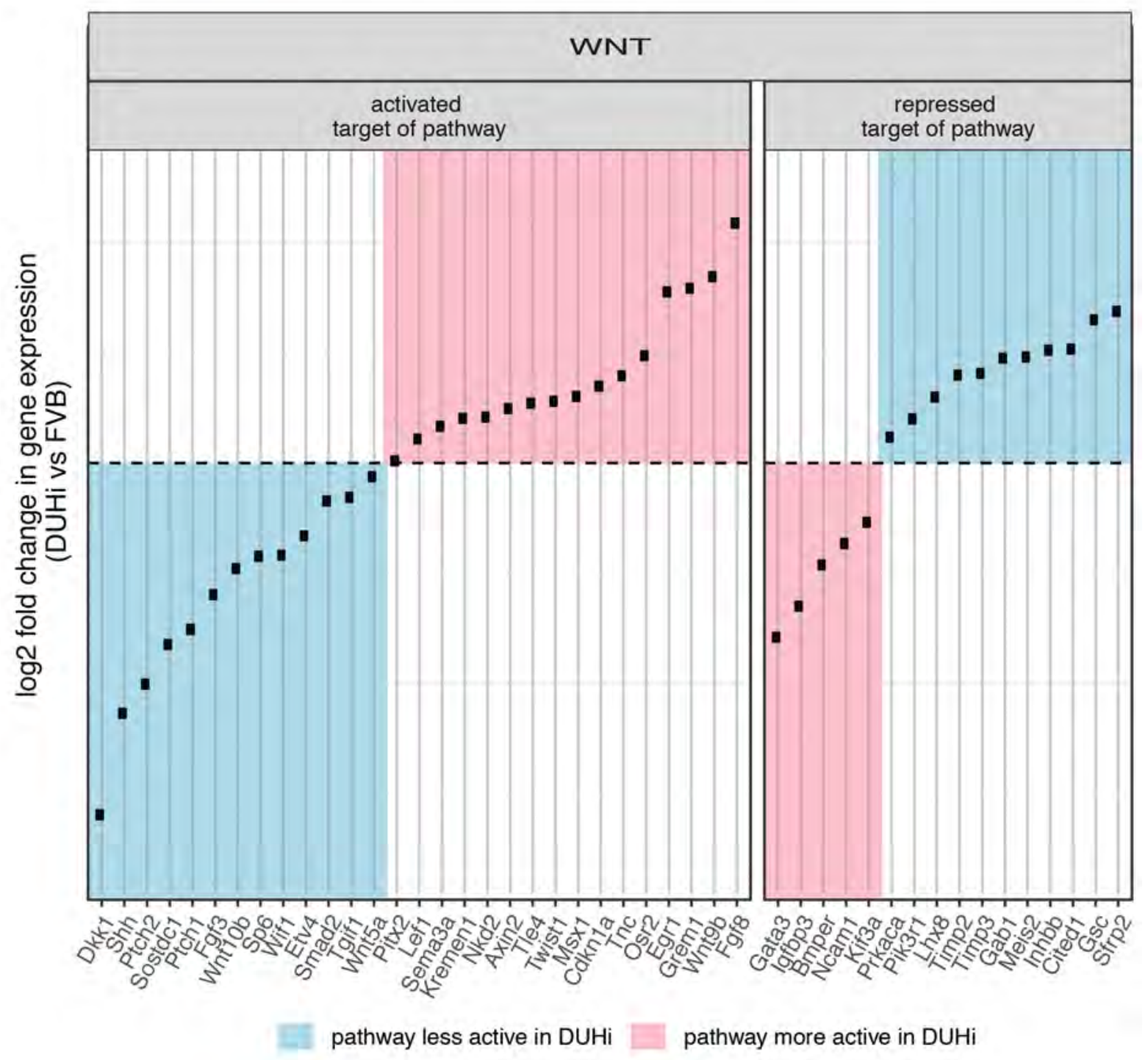

Supplementary Figure 9: comparative transcriptomics of DUHi versus FVB cap stage tooth germs. (A-B) Differential expression of sostdc1 (A) and sprouty2 (B) in an RNAseq experiment for lower and upper molar germs of FVB and DUHi mice. For each strain, we sampled 3 embryos from the same litter with slightly different body weight (DUHi (embryo weight: 196, 219 and $239 \mathrm{mg}$ ) and FVB $(195,215$ and $233 \mathrm{mg})$. This corresponds to slightly different embryonic ages, from the youngest age for the lightest embryo to the oldest age for the heaviest embryo. For each gene, the bars show normalized RNAseq counts (Basemean, 
DESeq2) in the lower (low) and upper (up) molar germs of these 3 DUHi and 3 FVB embryos. The test for differential expression was performed between all DUHi versus FVB samples, taking jaw into account as a factor in DEseq2. Sprouty2: fold change in DUHi=1.3, padj=2.4.E-8; Sostdc1/Ectodin: fold change in $\mathrm{DUHi}=1.8$; padj=0.02. (C) A panel showing log fold change in expression level in DUHi/FVB (positive fold change: higher expression level in DUHi; negative fold change: lower expression level in DUHi) for Wnt target genes (activated or repressed by Wnt pathway), that are differentially expressed between the two strains (see supplementary text for details). In blue, targets arguing for lesser Wnt activity in DUHi mice $(n=24)$. In pink, targets arguing for greater Wnt activity in DUHi mice $(n=21)$.
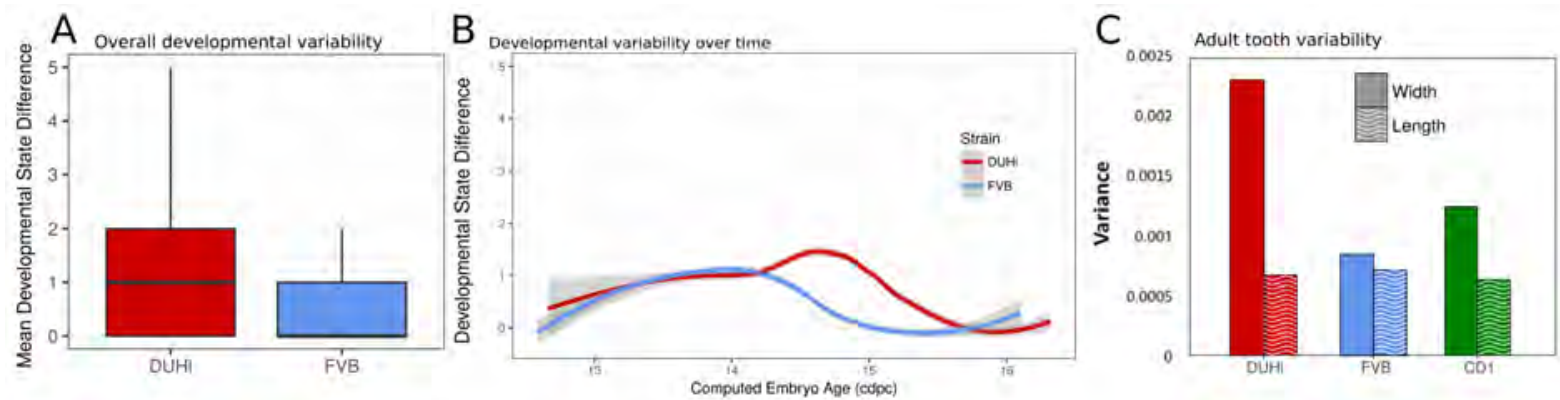

\section{Supplementary Figure 10: Developmental and adult variability in lower molars}

(A) A measure of developmental variation in the developing lower molars of FVB and DUHi strains. The figure shows a boxplot of developmental state differences calculated for pairs of samples with less than 0.25 difference in computed embryonic age (cdpc). Samples close in age are significantly more different in developmental state in DUHi versus FVB mice, according to a Wilcoxon test $(p<0.00001)$. See the material and methods for further explanation on this measure of developmental variation. (B) The mean developmental distance between nearby samples (computed embryonic age difference $<0.25$ ) is plotted as the local regression line for both strains (standard deviation shown in grey). (C) Morphological variation in the adult first molar, measured as the variance in molar width and length. In contrast with the situation in the upper molar, the extent of variation in molar length is comparable in the lower first molars for the three strains (variation in lower molar width is however higher in DUHi and the outbred CD1 strain).

\section{Supplementary file 1: an excel file with RNAseq experiment data.}

- Sheet "All genes": data for all genes (basemean in 6 conditions, log fold change, p-adj for DE analysis comparing lower and upper molars in DUHi versus FVB samples)

- Sheet "DE genes": DE genes only 
- Sheet "BMP pathway": DE genes with regulatory information in the regulatory list established by O'Connell et al. 2012 paper (activated or repressed by BMP pathway) and the corresponding indication of weaker/greater activity.

- Sheet "Wnt pathway": DE genes with regulatory information in the regulatory list established by O'Connell et al. 2012 paper (activated or repressed by Wnt pathway) and the corresponding indication of weaker/greater activity. 


\section{Supplementary Materials \& Methods}

Bayesian method to estimate embryonic age for mouse embryos

See also: https://github.com/msemon/cdpc

\section{Background}

Embryonic age in mouse embryos can be crudely estimated using the number of days post coïtum (dpc, where noon of the day when a plug is detected is equal to 0.5 days). For an equivalent age in dpc, mouse embryos show considerable inter and intra-litter variability in embryonic age, despite the absence of genetic variation in inbred mouse strains. Reasons for this are environmental effects on the embryo that are either common to the whole litter (female physiology influencing the timing of ovulation or implantation, number of embryos implanted influencing the demand on the maternal physiology) or specific to the embryo (e.g. position in the uterus determining placental efficiency).

To study fine-scale dynamics of tooth development, we needed to estimate a numeric embryonic age. Embryo weight is well correlated with embryonic age (including tooth developmental stage, Peterka 2002) and can be used as a proxy. However, variability in embryo weight is not solely due to variability in embryonic age. Although very limited nutritional availability can slow down developmental rate, subtler limitations may only result in low body weight embryos, as compared to others with the same embryonic age.

Reciprocally, exceptionally good nutritional conditions should not speed up developmental rate, but rather result in high body weight of embryos, as compared to others with the same developmental stage. In late stages, sex influences body weight (males are heavier). In our practice, we noticed that embryos and/or litters that deviate from the expected body weight for their age in dpc have more chances to be mis-estimated from their body weight only. This is the case for embryos displaying extreme body weight within a litter distribution at a given age in dpc. This is also the case for litters for which body weight poorly matches with dpc.

Therefore, we wish to build a model that could estimate the embryonic age from the body weight and dpc, taking into account intra and inter litter variations.

\section{Construction of the model}


We built a model to improve embryonic age estimation based on observed values of embryo weight, embryo litter, and age in dpc (for the litter).

First, we consider a basic model (a deterministic one) where the embryonic age of embryos would be equal to their age in dpc. We assume that there is a log-linear relationship between weight and dpc specific to each strain (FVB or DUHi). The slope and offset of this relationship are estimated from the data for each strain separately and termed respectively " $b$ " and " $a$ " in the model. The body weight in litter " $i$ " can then be modeled as in [1]

weight.in. $\log _{\mathrm{i}}=\mathrm{a}+\mathrm{b} *$ age.in.dpc $\mathrm{c}_{\mathrm{i}}$

In practice, age.in.dpc is not a precise measure of embryonic age. Different litters at the same age in dpc may be more or less developmentally advanced (inter-litter developmental effect) [2]. This induces a variability on weight.in.log in model [1] which is described in the model [2] by the addition of a stochastic term, eps.litter.dev, following a Gaussian distribution centered on 0 with a standard deviation, sd.litter.dev, that will be estimated from data.

weight.in. $\log _{\mathrm{i}}=\mathrm{a}+\mathrm{b} *$ age.in.dpc $\mathrm{c}_{\mathrm{i}}+$ eps.litter.dev $\mathrm{i}_{\mathrm{i}}$

However, different litters at the same age in dpc, and at the same embryonic age, may have different mean weights because each pregnancy will provide a specific environment to the embryos. The addition of another stochastic term in the model, eps.litter.preg, is needed to account for this pregnancy effect [3]. We assume that this effect follows a Gaussian distribution centered on 0 with a known standard deviation (sd.litter.preg). From our practice we consider two values for sd.litter.preg : 0.05 and 0.1 . The first one (0.05) corresponds to a $95 \%$ fluctuation interval of eps.litter.preg of $[-0.1 ; 0.1]$ which gives in the ratio of weight [$10 \% ; 10 \%]$, so to a realistic maximum effect on weight of $20 \mathrm{mg}$ for a $200 \mathrm{mg}$ embryo. The second value (0.1) corresponds to a 95\% fluctuation interval of eps.litter.preg of $[-0.2 ; 0.2]$ which corresponds in the ratio in weight of $[-18 \% ; 22 \%]$ so to an excessive maximum effect on weight of $40 \mathrm{mg}$ for a $200 \mathrm{mg}$ embryo.

weight.in. $\log _{\mathrm{i}}=\mathrm{a}+\mathrm{b} *$ age.in.dpc $\mathrm{c}_{\mathrm{i}}+$ eps.litter.dev $\mathrm{i}+$ eps.litter.preg $\mathrm{i}_{\mathrm{i}}$ 
The previous complexifications of the model are drawn at the level of the litter, presuming that all embryos of a given litter were at the same embryonic age. However, we know that 1) all embryos are not at the same embryonic age and 2) within a litter, weight is a very good indicator of relative embryonic age. To take advantage of this, we modeled that within a litter, embryonic age follows a Gaussian distribution, centered on the mean stage of the litter. So to describe the weight of the embryo $j$ in the litter $i$, we add to the model [4] a last term, eps.embryo.dev, following a Gaussian distribution centered on 0 with a standard deviation of sd.embryo.dev estimated from the data and characterizing intra-litter variability on body weight.

Weight.in. $\log _{\mathrm{ij}}=\mathrm{a}+\mathrm{b} *$ age.in.dpc $\mathrm{c}_{\mathrm{i}}+$ eps.litter.dev $\mathrm{i}_{\mathrm{i}}+$ eps.litter.preg $\mathrm{i}_{\mathrm{i}}+$ eps.embryo.dev $_{\mathrm{ij}}$ [4]

\section{Estimation of embryonic age}

Once the model fitted, the development age was estimated from model 4 just by removing the pregnancy effect eps.litter.pregi and inverting the relation :

age.devij $=($ weight.in.logij - eps.litter.pregi $-a) / b$

Note that this estimation of the embryonic age (age.devij) from the body weight in log of each embryo (weight.in.logij) requires the knowledge of parameters $a$ and $b$ and of random effects due to pregnancy for each litter (eps.litter.pregi). Those were previously estimated from data as explained below.

\section{Estimation of the parameters}

Parameters a, b, sd.litter.dev, sd.embryo.dev and random effects of the model were estimated from data in two scenarios for two fixed sd.litter.preg values $(0.05$ in the realistic scenario and 0.10 in the excessive scenario) as it is not possible to dissociate only from data the two components of inter-litter variability : variability in weight due to embryonic age and to pregnancy. Vague uniform priors were assigned to the other parameters (a, b, sd.litter.dev, sd.embryo.dev) allowing variation of each within a realistic range (see Supplementary Methods Table 1) 
Monte Carlo Markov-Chain (MCMC) techniques were used to estimate the joint posterior distribution of parameters from prior distributions and data. Computations were performed using the JAGS software via the R package rjags (Plummer et al., 2016) (a runnable R script is provided in Supplementary material with data corresponding to strain FVB). Three independent MCMC chains were run in parallel. For each chain, 110,000 samples were produced. The first 10,000 were considered as burn-in phase and discarded. To avoid autocorrelation, the remaining 100,000 samples were thinned by selecting one out of 20 samples, thus keeping 5000 samples per chain. We checked the convergence again by displaying MCMC chain traces and autocorrelation plots and by computing the Gelman and Rubin's statistics as modified by Brooks and Gelman (Brooks and Gelman, 1998). For each parameter, its point estimate was defined as the median of its marginal posterior distribution, and the $95 \%$ credible interval was defined from the 2.5 and 97.5 percentiles of this distribution. The calculation of the development age for each embryo was integrated in the model was thus estimated in the same way from its posterior distribution estimated by MCMC.

Supplementary Methods Table 1. Medians and 95\%credibility intervals for the parameters for each strain, and each scenario (termed realistic for sd.litter.preg $=0.05$, and permissive for sd.litter.preg $=0.1$ in the text).

A: DUHi, permissive model

\begin{tabular}{|l|l|l|l|}
\hline DUHi & \multicolumn{3}{|l|}{ sd.litter.preg $=0.1$} \\
\hline & $2.5 \%$ & $\begin{array}{l}50 \% \\
\text { (median) }\end{array}$ & $97.5 \%$ \\
\hline a & 5.3937 & 5.4742 & 5.5531 \\
\hline b & 0.4816 & 0.5402 & 0.6065 \\
\hline sd.embryo.dev & 0.1556 & 0.1695 & 0.1855 \\
\hline sd.litter.dev & 0.1376 & 0.1997 & 0.2889 \\
\hline
\end{tabular}

B: DUHi, realistic model

\begin{tabular}{|l|l|l|l|}
\hline DUHi & \multicolumn{3}{|l|}{ sd.litter.preg $=0.05$} \\
\hline & $2.5 \%$ & $50 \%$ & $97.5 \%$ \\
\hline $\mathrm{a}$ & 5.3928 & 5.4732 & 5.5555 \\
\hline $\mathrm{b}$ & 0.4809 & 0.5391 & 0.6050 \\
\hline
\end{tabular}




\begin{tabular}{|l|l|l|l|}
\hline sd.embryo.dev & 0.1557 & 0.1694 & 0.1854 \\
\hline sd.litter.dev & 0.1632 & 0.2188 & 0.3003 \\
\hline
\end{tabular}

C: FVB, permissive model

\begin{tabular}{|l|l|l|l|}
\hline FVB & \multicolumn{3}{|l|}{ sd.litter.preg $=0.1$} \\
\hline & $2.5 \%$ & $50 \%$ & $97.5 \%$ \\
\hline a & 5.5007 & 5.5689 & 5.6378 \\
\hline b & 0.4057 & 0.4622 & 0.5173 \\
\hline sd.embryo.dev & 0.0812 & 0.0875 & 0.0946 \\
\hline sd.litter.dev & 0.1549 & 0.2032 & 0.2683 \\
\hline
\end{tabular}


D : FVB, realistic model

\begin{tabular}{|l|l|l|l|}
\hline FVB & \multicolumn{3}{|l|}{ sd.litter.preg $=0.05$} \\
\hline & $2.5 \%$ & $50 \%$ & $97.5 \%$ \\
\hline a & 5.5019 & 5.5697 & 5.6378 \\
\hline b & 0.4060 & 0.4611 & 0.5175 \\
\hline sd.embryo.dev & 0.0813 & 0.0875 & 0.0944 \\
\hline sd.litter.dev & 0.1774 & 0.2211 & 0.2817 \\
\hline
\end{tabular}




\section{Comparison of age measurements}

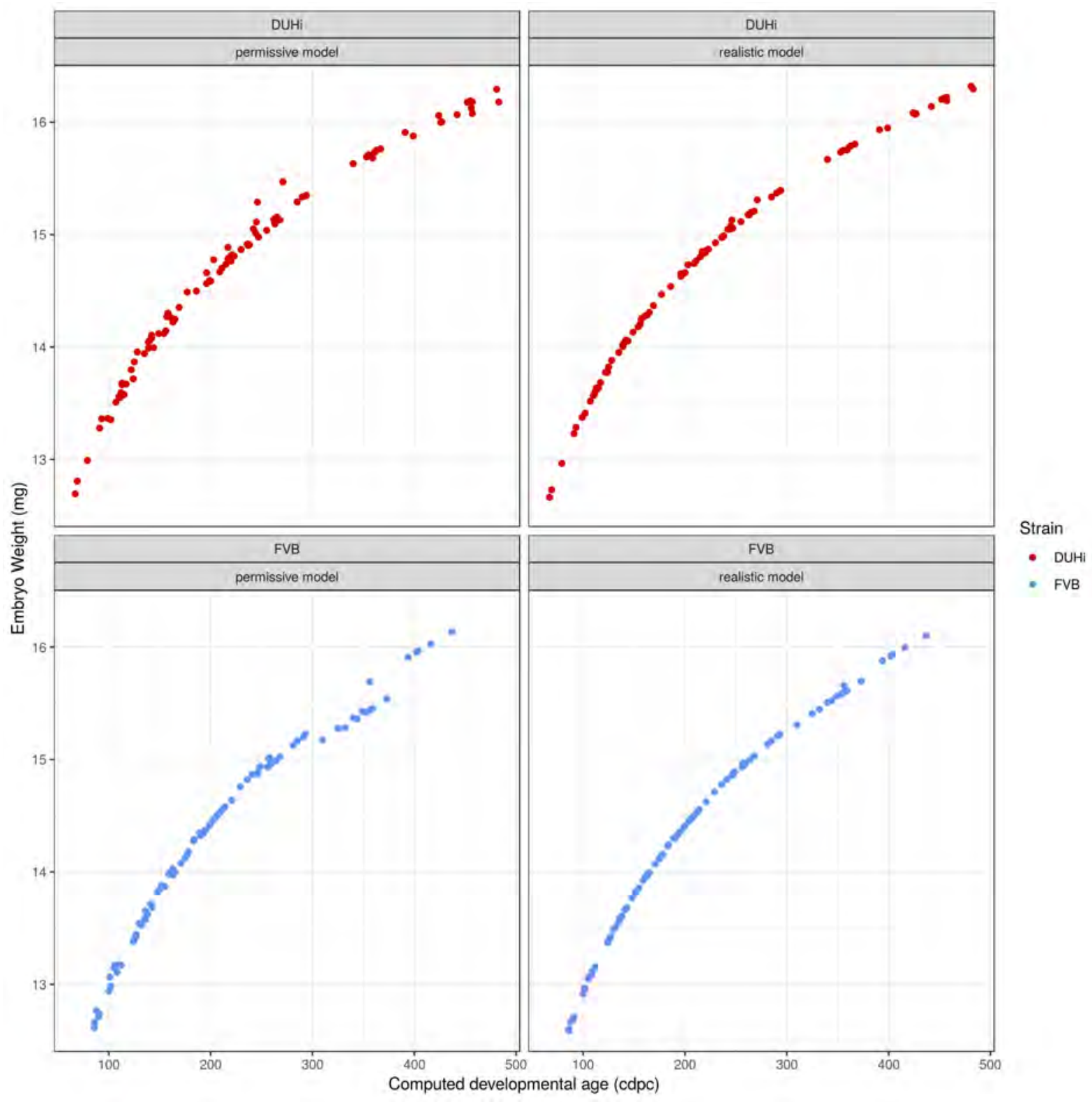

Supplementary Methods Figure 1:

Comparison between two versions of computed embryonic age (realistic versus permissive), as plotted against embryo weight (mg). DUHi (red) and FVB (blue) samples are shown in separate panels. 


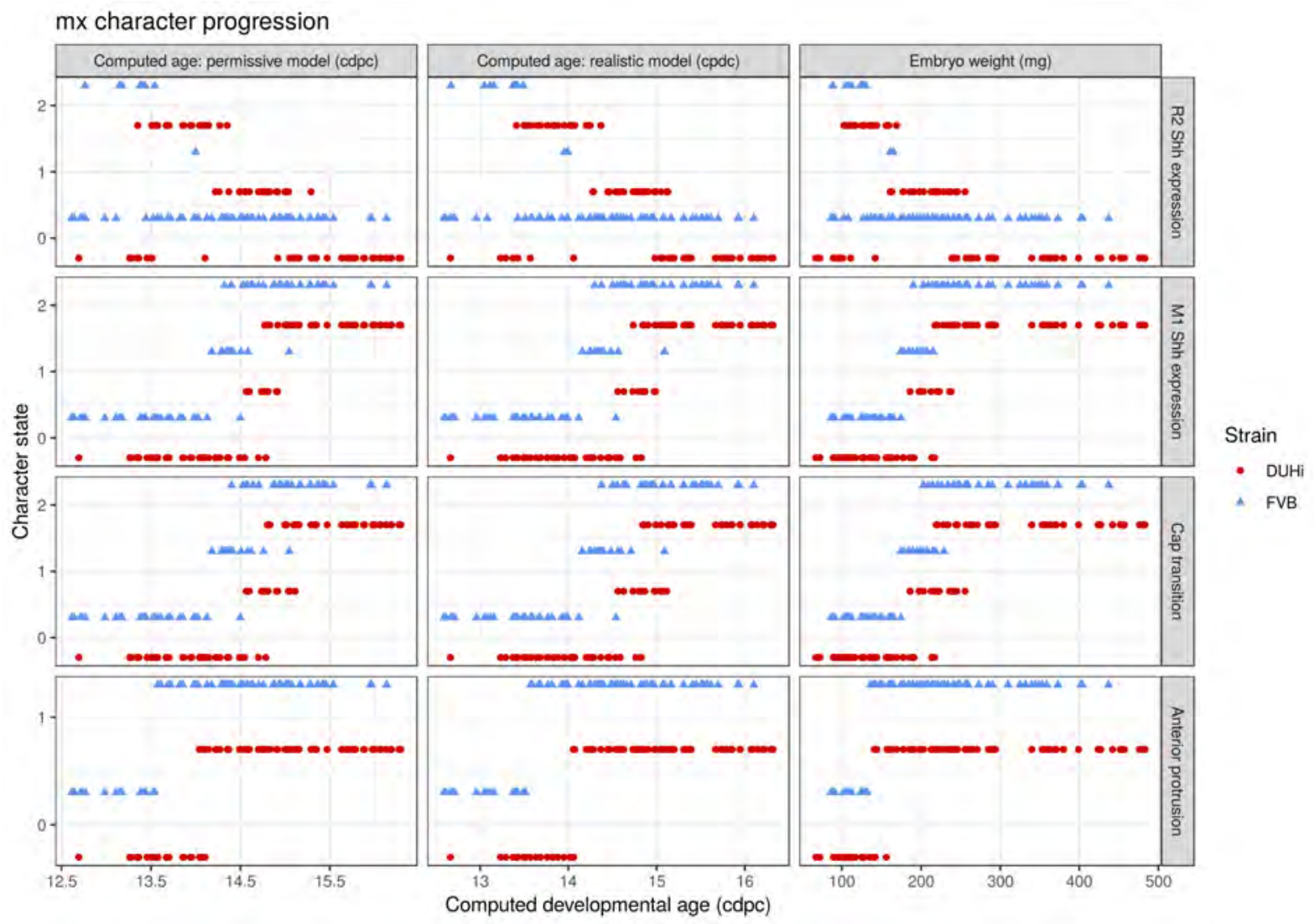

Supplementary Methods Figure 2: Comparative progression of scored characteristics in upper molar epithelia under different measures of age

Progression of four characters (R2 Shh expression, M1 Shh expression, cap transition and anterior protrusion of the dental epithelium) is depicted in samples of upper molars from two strains (FVB and DUHi). In order to allow the comparison of age measurements, the temporal axis is provided by computed embryonic age $\left({ }_{c} \mathrm{dpc}\right)$ under the realistic and permissive parameters and by embryo weight as an age proxy. All samples were scored using the criteria provided in Supplementary Table 1. 


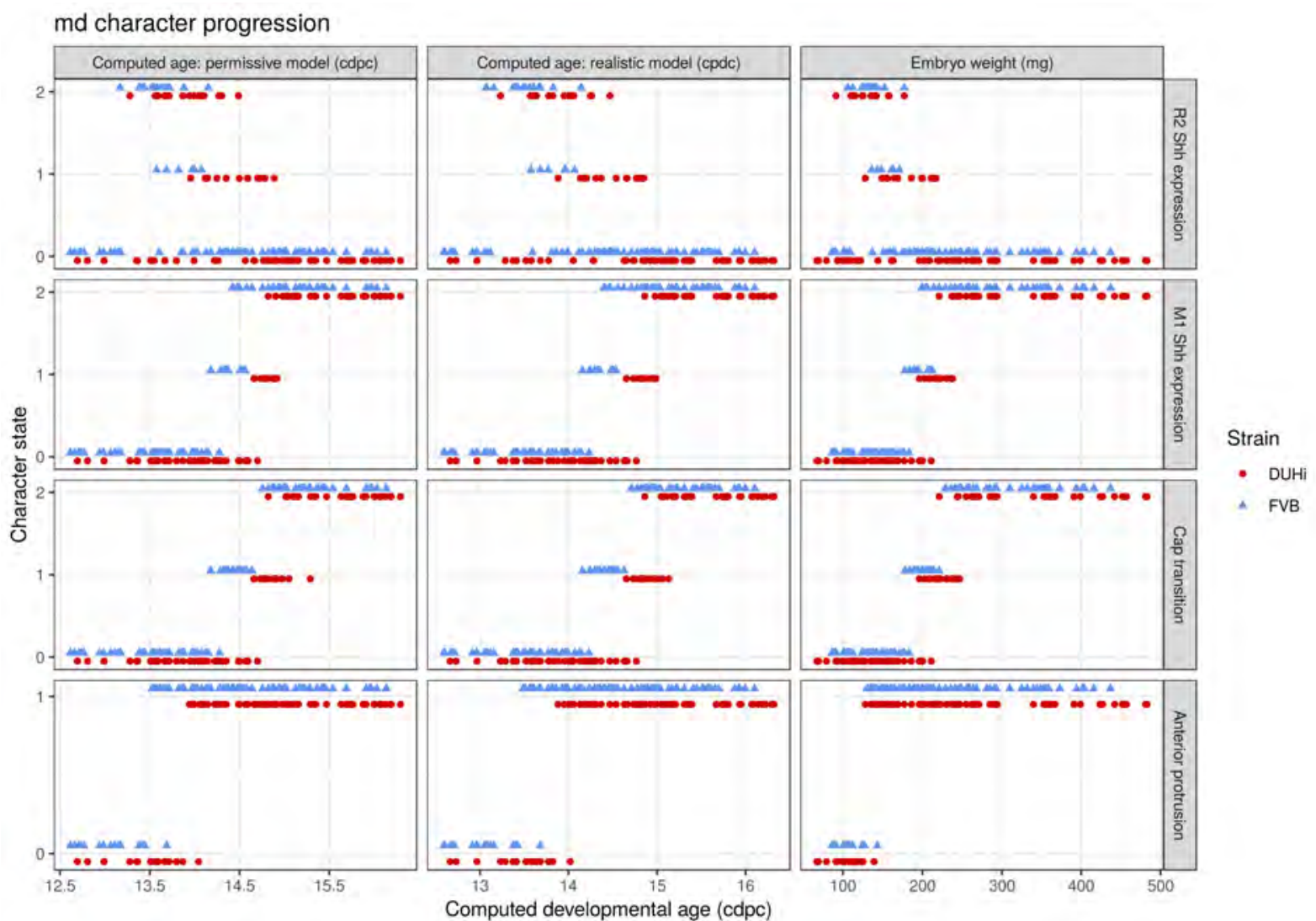

Supplementary Methods Figure 3: Comparative progression of scored characteristics in

\section{lower molar epithelia under different measures of age}

Progression of four characters (R2 Shh expression, M1 Shh expression, cap transition and anterior protrusion of the dental epithelium) is depicted in samples of lower molars from two strains (FVB and DUHi). In order to allow the comparison of age measurements, the temporal axis is provided by computed embryonic age $\left({ }_{c} \mathrm{dpc}\right)$ under the realistic and permissive parameters and by embryo weight as an age proxy. All samples were scored using the criteria provided in Supplementary Table 1. 


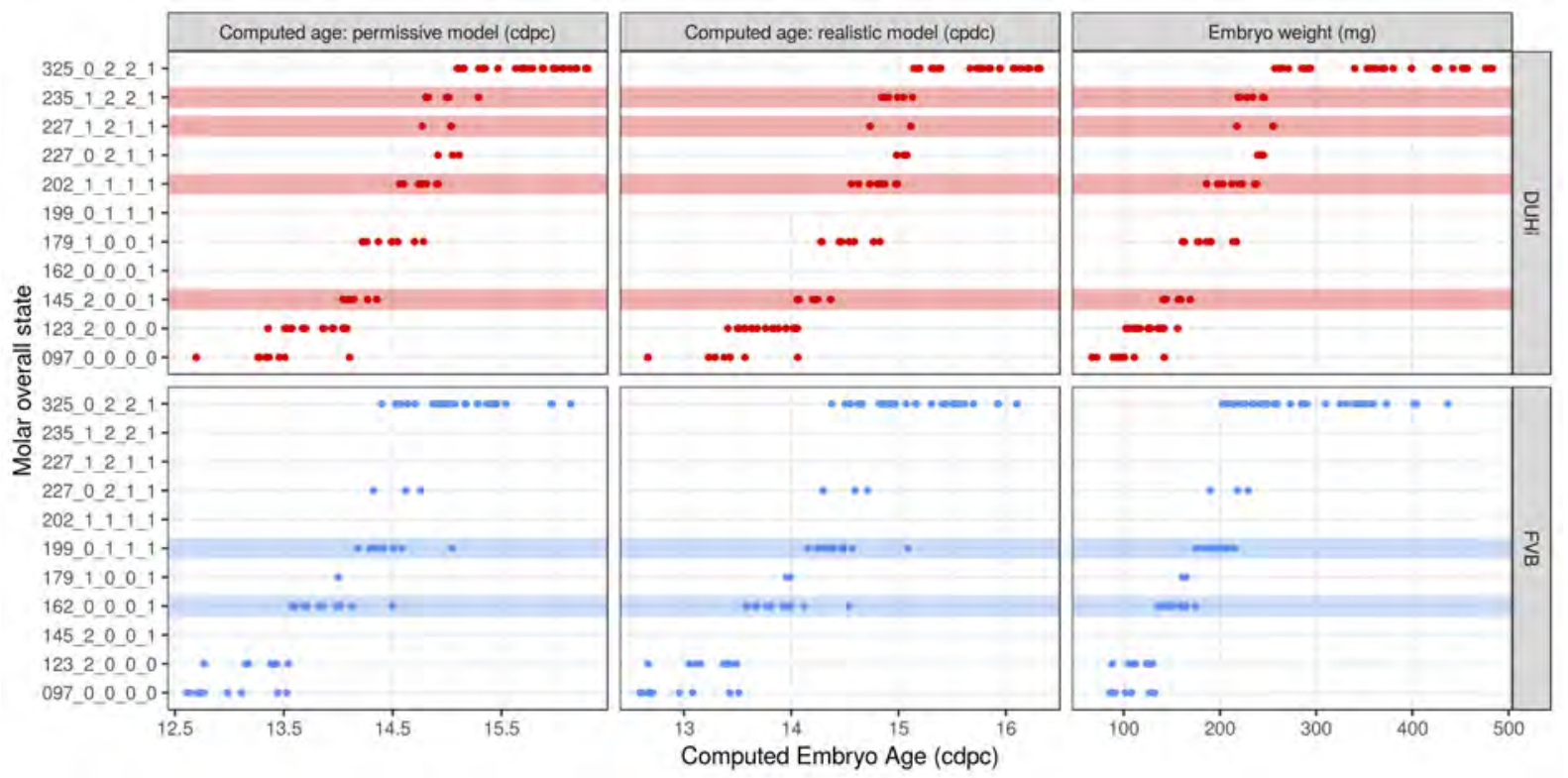

\section{Supplementary Methods Figure 4: Total state of embryonic upper molars under}

\section{different measures of age}

Temporal distribution of developmental state of the developing upper molar, produced by combining a value for each of the four scores for a given sample, based on criteria from Supplementary Table 1. Each of the developmental states observed are shown and are ordered according to the average embryonic weight of the samples within that group. Each state present is coloured according to whether it is found in DUHi only (red), in FVB only (blue), or in both DUHi and FVB (white) samples. In order to allow the comparison of age measurements, the temporal axis is provided by computed embryonic age $\left({ }_{c} \mathrm{dpc}\right)$ under the realistic and permissive parameters and by embryo weight as an age proxy. 

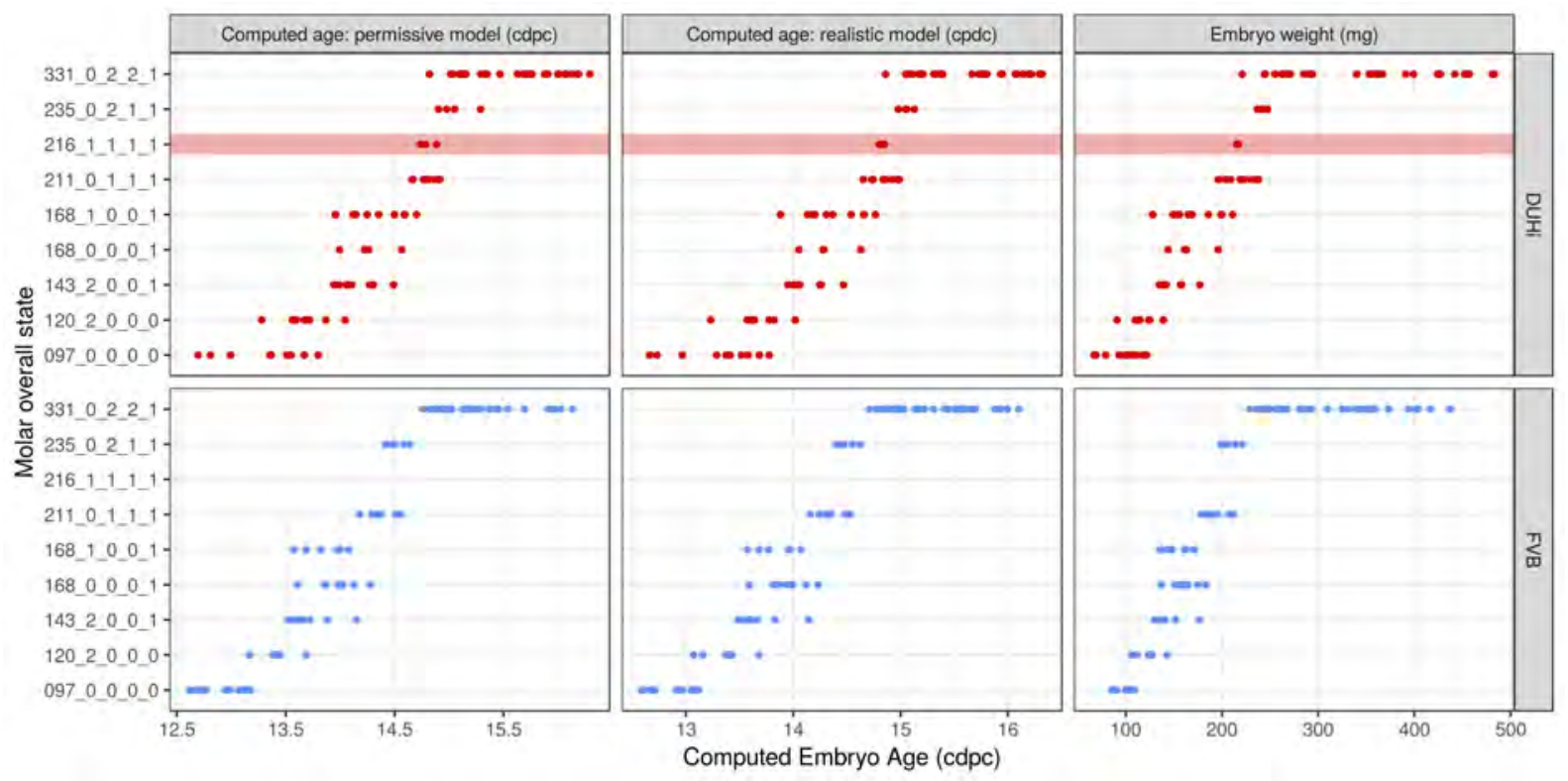

\section{Supplementary Methods Figure 5: Developmental state of embryonic lower molars under different measures of age}

Temporal distribution of developmental state of the developing lower molar, produced by combining a value for each of the four scores for a given sample, based on criteria from Supplementary Table 1. Each of the developmental states observed are shown and are ordered according to the average embryonic weight of the samples within that group. Each state present is coloured according to whether it is found in DUHi only (red) or in both DUHi and FVB (grey) samples. In order to allow the comparison of age measurements, the temporal axis is provided by computed embryonic age $\left({ }_{c} \mathrm{dpc}\right)$ under the realistic and permissive parameters and by embryo weight as an age proxy. 

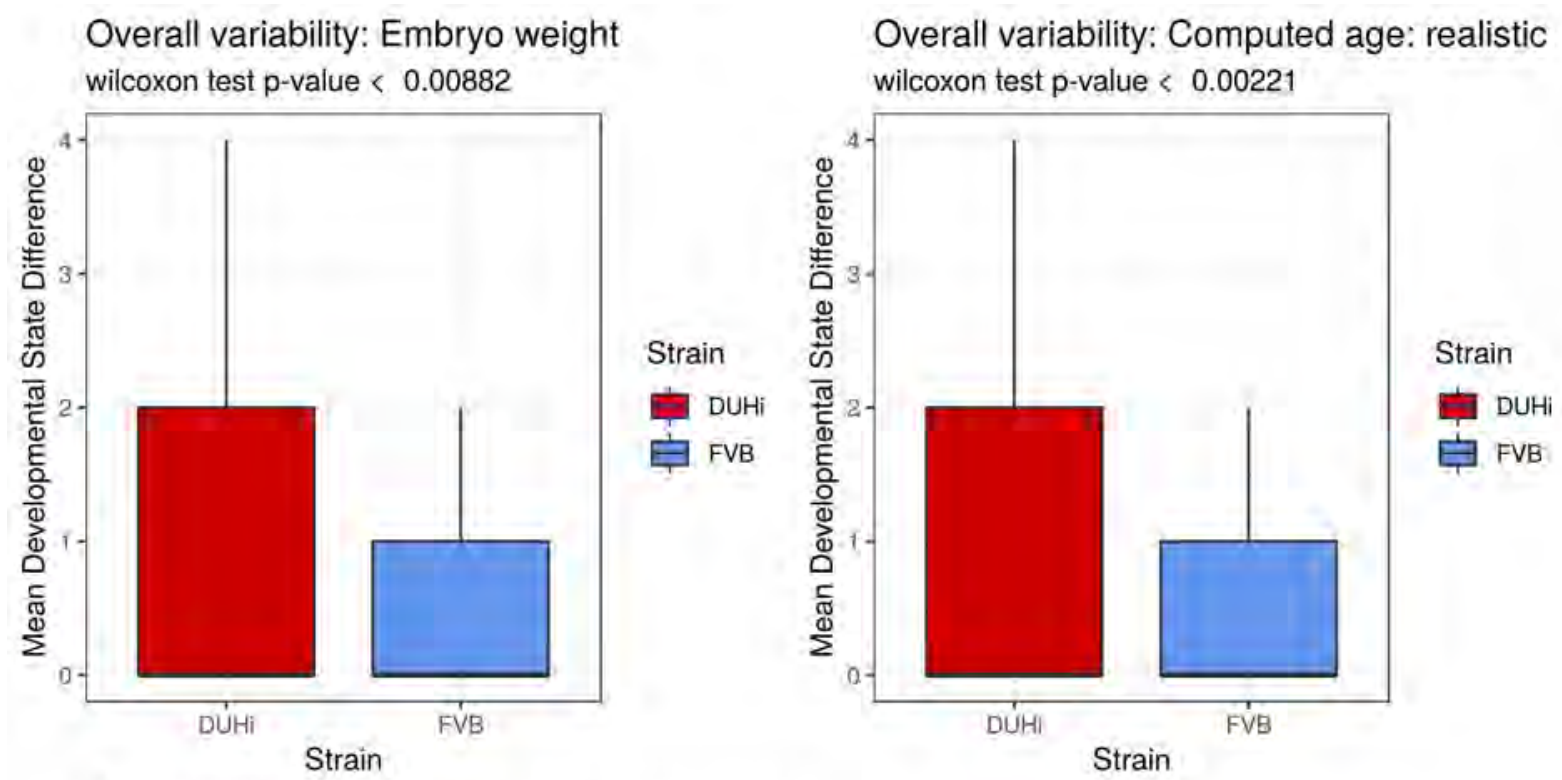

Overall variability: Computed age: permissive wilcoxon test p-value $<1 e-05$

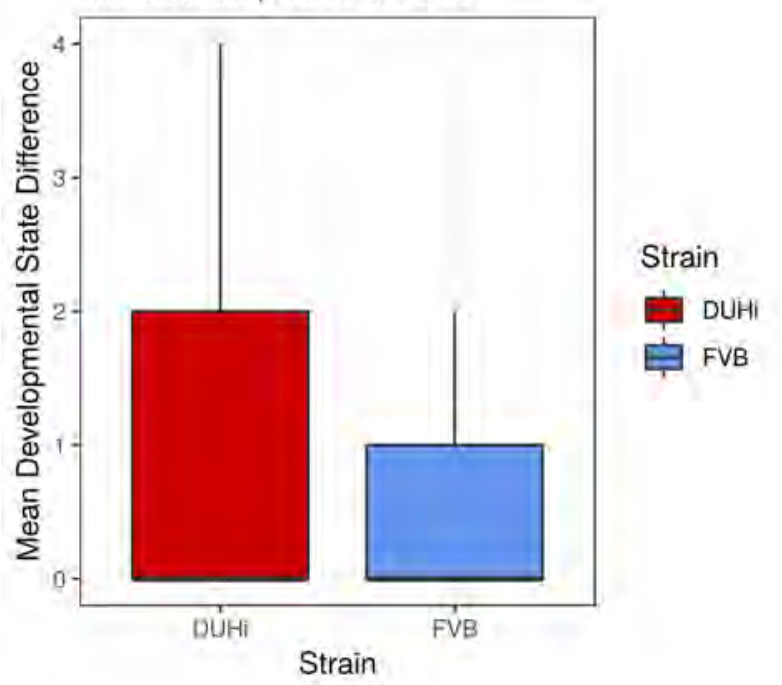

\section{Supplementary Methods Figure 6: Developmental variability in the developing upper} molars of DUHi and FVB mice embryos under different measures of age

Variability in the developing upper molar is represented as a boxplot of developmental state differences calculated for pairs of samples with less than 0.25 difference in computed embryonic age $\left({ }_{c} \mathrm{dpc}\right)$. Samples close in age are significantly more different in developmental state in DUHi versus FVB mice when the temporal axis is provided by computed embryonic age ( $(\mathrm{dpc})$ under the realistic and permissive parameters and when embryo weight is used as an age proxy. 

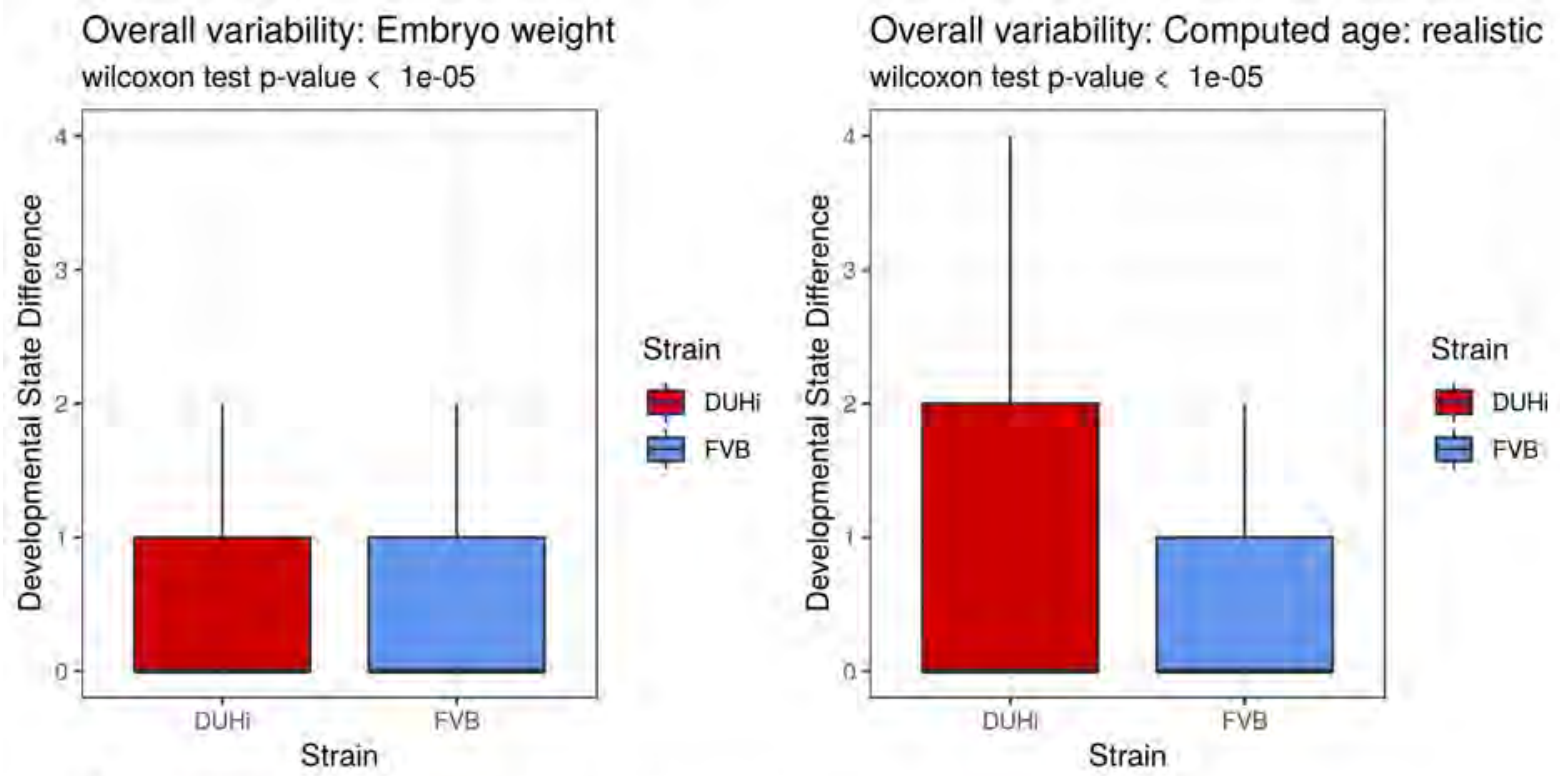

Overall variability: Computed age: permissive wilcoxon test p-value $<1 \mathrm{e}-05$

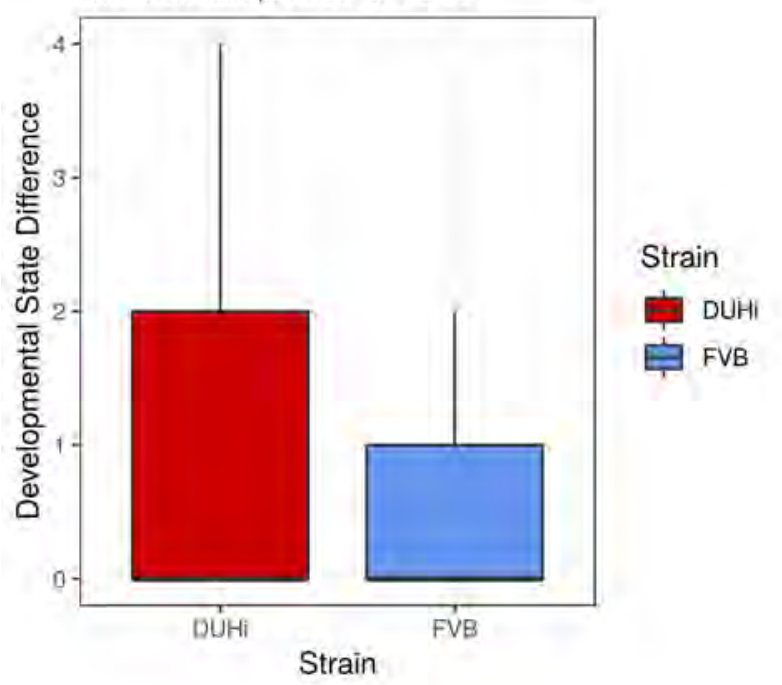

Supplementary Methods Figure 7: Developmental variation in the developing lower molars of DUHi and FVB mice embryos under different measures of age

Variation in the developing lower molar is represented as a boxplot of developmental state differences calculated for pairs of samples with less than 0.25 difference in computed embryonic age $\left({ }_{c} d p c\right)$. Samples close in age are significantly more different in developmental state in DUHi versus FVB mice when the temporal axis is provided by computed embryonic age $\left({ }_{c} \mathrm{dpc}\right)$ under the realistic and permissive parameters. 

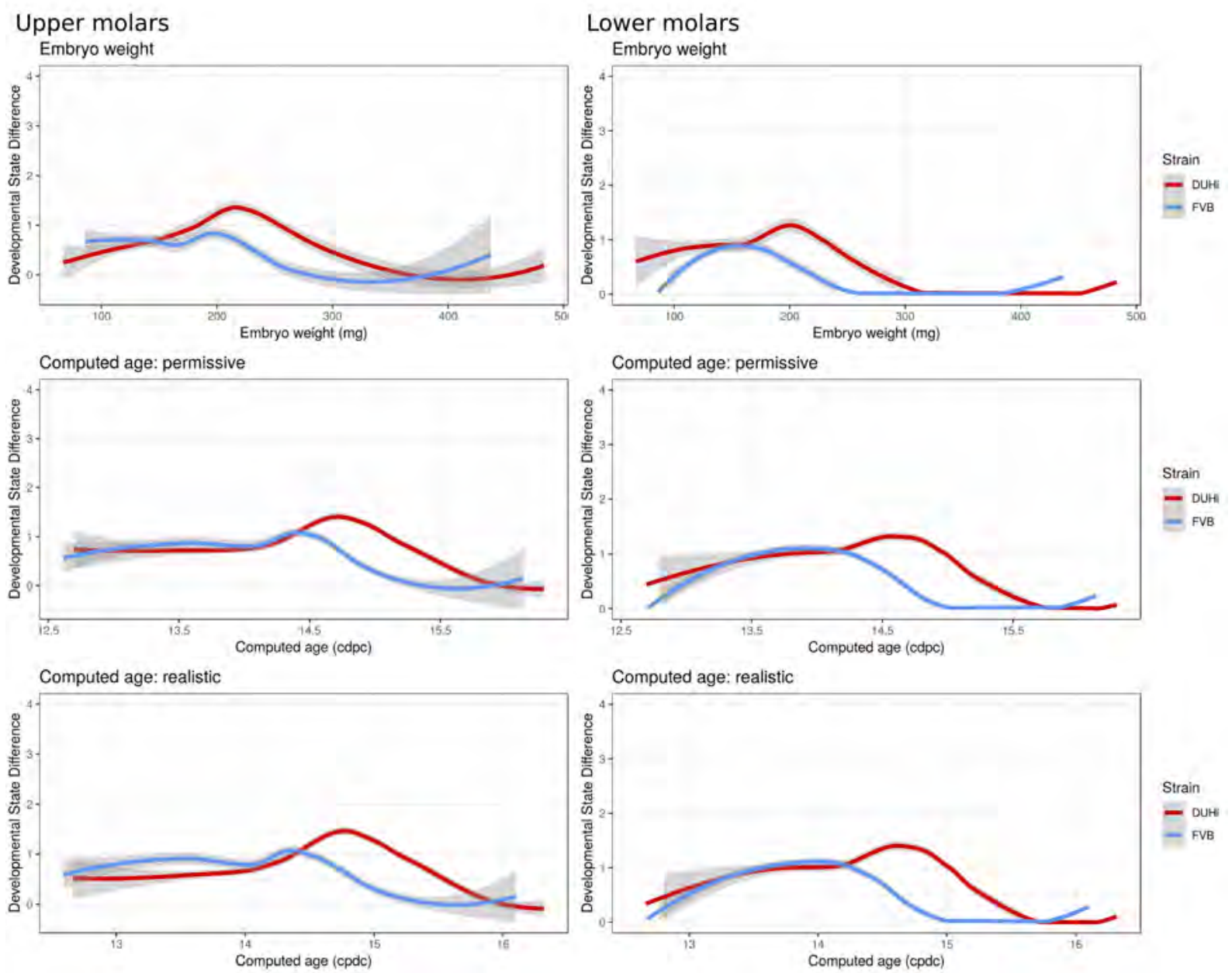

Supplementary Methods Figure 8: Developmental variation over time in the upper and lower molars of DUHi and FVB mice embryos under different measures of age

The mean developmental distance between nearby samples (embryonic age difference $<$ $0.25 \mathrm{~d}$ ) is plotted as the local regression line (LOESS smoothing) for both strains (standard deviation shown in grey). In order to allow the comparison of age measurements, the temporal axis is provided by computed embryonic age $\left({ }_{c} \mathrm{dpc}\right)$ under the realistic and permissive parameters and by embryo weight as an age proxy. Developmental variation is higher in DUHi under all measures of age, and peaks at time of R2 and M1 signaling center coexistence. 
bioRxiv preprint doi: https://doi.org/10.1101/818484; this version posted October 24,2019 . The copyright holder for this preprint (which

was not certified by peer review) is the author/funder. All rights reserved. No reuse allowed without permission. 